\title{
The breaking of geometric similarity
}

DOI:

10.1016/j.jjmecsci.2020.105925

\section{Document Version}

Accepted author manuscript

Link to publication record in Manchester Research Explorer

\section{Citation for published version (APA):}

Davey, K., Darvizeh, R., Golbaf, A., \& Sadeghi, H. (2020). The breaking of geometric similarity. International Journal of Mechanical Sciences, 187, [105925]. https://doi.org/10.1016/j.ijmecsci.2020.105925

\section{Published in:}

International Journal of Mechanical Sciences

\section{Citing this paper}

Please note that where the full-text provided on Manchester Research Explorer is the Author Accepted Manuscript or Proof version this may differ from the final Published version. If citing, it is advised that you check and use the publisher's definitive version.

\section{General rights}

Copyright and moral rights for the publications made accessible in the Research Explorer are retained by the authors and/or other copyright owners and it is a condition of accessing publications that users recognise and abide by the legal requirements associated with these rights.

\section{Takedown policy}

If you believe that this document breaches copyright please refer to the University of Manchester's Takedown Procedures [http://man.ac.uk/04Y6Bo] or contact uml.scholarlycommunications@manchester.ac.uk providing relevant details, so we can investigate your claim.

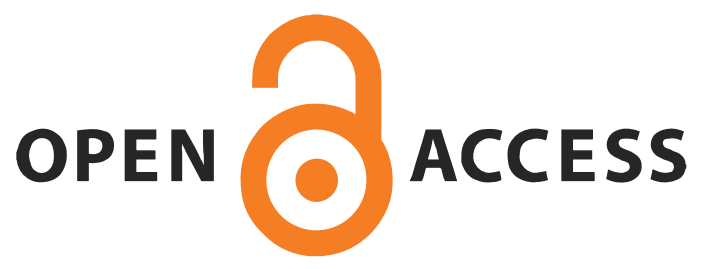




\title{
THE BREAKING OF GEOMETRIC SIMILARITY
}

\author{
*Keith Davey ${ }^{\mathrm{a}, \mathrm{b}}$, Rooholamin Darvizeh ${ }^{\mathrm{a}}$, Ali Golbaf ${ }^{\mathrm{c}}$, Hamed Sadeghic

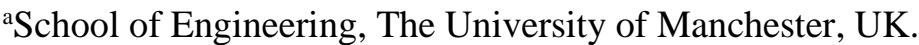 \\ ${ }^{\mathrm{b}}$ Advanced Forming Research Centre, University of Strathclyde, UK. \\ 'Department of Mechanical Engineering, University of Guilan, Rasht, Iran.
}

\begin{abstract}
Scaled experimentation plays an important role in prototype and process development but is recognised to be severely constrained by the need for similitude, founded on the concepts of geometric, kinematic and kinetic similarity.

This paper examines the possibility of breaking the requirement for geometric similarity by introducing the law of finite similitude for anisotropic scaling, which applies to continuum mechanics on anisotropically-scaled spaces. The law confirms that similarity solutions on skewed spaces always exist separately for quasistatic deformational and thermal-continuum problems. Thermomechanical and continuum dynamic problems however are shown to suffer from the inclusion of a (non-physical) non-orthogonal metric arising from the skewed coordinate system associated with anisotropic-space scaling. Even in this case however, an important subclass of problems is shown to be physically realisable involving a dominant component of velocity (displacement), where geometric similarity can again be broken yet retain good accuracy. The ability to skew artefacts yet achieve similitude is recognised to be a particularly important outcome as it allows for example one experimental model to be used to predict the behaviour of multiple skewed models.

To showcase and highlight the significance of the new concept various scaled numerical models with anisotropic scaling with different geometrical scaling ratios in different directions is considered. The applicability of the theory is tested on a number of case studies providing strong supporting evidence for the validity and applicability of the new theory.
\end{abstract}

Keywords: finite similitude; scaled experimentation; scaled models; anisotropic scaling.

*Corresponding author. Tel.: +44(0)161306 3834.

E-mail address: keith.davey@manchester.ac.uk. 


\section{INTRODUCTION}

The idea of similarity is a concept over a century old and is founded on the notion that physics is essentially dimensionless. The benefit of dimensionless physics is patent and gives for example laboratory-scaled experiments relevance in the real-world applications. It facilitates commercial enterprises to explore and investigate the performance of potential prototypes and processes by the means of scaled models. A key underpinning theorem of moderndimensional analysis is the Buckingham Pi theorem [1-2], which formalises Rayleigh's method of dimensional analysis [3]. The basic idea underpinning the theorem is that any physical equation involving $n$ physical variables can be recast in terms of $n-k$ dimensionless parameters $\pi_{1}, \pi_{2}, \ldots, \pi_{n-k}$. Here $k$ is the number of physical dimensions and is the rank of the dimensional matrix [4]. The strength but also the weakness of the approach is that the precise physical equation can be unknown, which may be of benefit for curve-fitting experimental results but provides no information on the limits of the underpinning equation involved. Another weakness of the approach is that the so called Pi groups $\left\{\pi_{i}\right\}$ are not unique and a certain degree of physical expertise is required to establish meaningful groups. Related to this approach however is direct dimensional analysis applied to the governing equations typically in the form of partial differential equations (PDEs) [5]. Casting the governing PDEs in a dimensionless form reveals dimensionless parameters as coefficients in the recast equations. This approach can be particularly advantageous in approximation theory (e.g. singular perturbation theory) arising out of the ability to contrast the relative importance of terms in the PDEs. The magnitude of dimensionless parameters can be compared (being dimensionless and part of a total-ordered set) and small terms can often be neglected in the governing PDEs [6-9]. The simplest situation is the reduction of a parameter set to a single non-dimensional parameter using dimensional analysis. An example of this is the nondimensional scaling ratio governing the relative contribution of electrical and viscous fluid forces on the system behaviour identified by Zohdi [10]. A slightly more involved situation is explored by Smith [11] concerned with the enforcement of geometric similarity and maximum failure-load prediction in quasi-brittle materials and its dependence on scale. A particular weakness of dimensional analysis however is that in reality it is seldom possible to find similarity solutions. Pi groups can be formed but when contrasting the behaviour of systems at different scales it is rarely possible to form groups with identically the same magnitudes [8, 12-15]. Although it may be possible on occasions, when a particular subset of physics dominates, to neglect these differences, the breakdown in the approach is undoubtedly a limiting factor. An additional difficulty with the approach is down to the limited scales over which the governing equations apply. If the governing equations do not apply over the scales of interest, then there can be little expectation that dimensional approaches will be successful. The limiting factor in continuum physics is predominantly the constitutive laws invoked, which are designed to represent behaviours at the microscale on the macroscale. Thus, as a consequence of an inability to scale microscale physics, dimensional analysis involving constitutive laws has a high likelihood of failure. This type of failure is apparent in solidmechanics applications for example involving large deformation and multi-crystalline materials [16-19].

To the best of the authors' knowledge, there is no literature on anisotropic scaling [20]. The three main axioms of geometric, kinematic and dynamic similitude [20] underpin the concept of similarity and impose restrictions on physical behaviour. Geometric similitude in particular necessitates that all dimensions are scaled with an identical ratio [20]. Such a constraint can be particularly restrictive in areas where anisotropic scaling would be beneficial, which includes scaling studies involving thin-walled structures. The only works found loosely related to anisotropic scaling are presented in Refs. [21-23]. Huntley showed in 
Ref. [21] that by refining the concept of dimension to include directed length dimensions leads to a match in the number of equations and unknowns in dimensional analysis. However, Huntley's extension has some serious limitations being unable to handle vector equations involving cross products and also the use of angles as variables. To remedy these deficiencies, Siano [22-23] proposed that the directed dimensions of Huntley be replaced by orientational symbols. The difficulty with both these extensions to dimensional analysis is they are not founded on any underpinning physical concept and simply serve to expand the argument set in dimensional analysis.

The present work aims to show that the first axiom of geometric similitude can be broken without loss of similitude in the sense that scaled behaviour remains representative of fullscale behaviour. In this sense the first axiom is shown not to be completely necessary and consequently existing scaling techniques can be extensively broadened to a wider range of applications. To achieve this, an alternative viewpoint is taken in this paper which contrasts the physics between two synchronised control volumes with one control volume residing in a scaled space. The physics on control volumes is described by transport equations in their integral form and this approach provides three immediate benefits. First, transport equations describe both conservation and non-conservation fundamental laws, which apply over a large range of length scales. Related to this is that the transport equations in their integral form do not necessarily involve constitutive laws, which are a major concern with PDEs. Third, transport equations describe the physics of a finite domain and no assumption is therefore necessary that the physics of concern applies at a continuum point or at length scales smaller than the smallest control volume considered. In order to apply transport equations it has been found necessary to tighten up the rules pertaining to the movement of the control volumes involved. Introduced in this paper is the law of volume, which relates the movement of a control volume (as described by a differential equation) to a transport equation for volume. This law provides the foundation on which the theory is built and leads ultimately to the law of finite similitude for anisotropic scaling. It transpires that physics on skewed spaces can be related, which is of fundamental importance as it permits greater flexibility in scaled experimentation than hitherto would have been thought possible.

The concept of space distortion and its influence on attached coordinate systems is considered in Section 2 along with the idea of internal and external observers, where it is recognised that scaled experimentation is viewed by the external observer unaffected by the scaling process.

The link between temporal and spatial differentials, volume measures and control volume movement is examined in Section 3. The transport equations for physical and trial (scaled) spaces are introduced in Section 4 along with the conditions necessary and sufficient for matching physics (as described by transport equations). The ten transport equations of interest are introduced in Section 5, which describe conservation rules for volume, mass, momentum and energy and non-conservation rules for movement and entropy. Satisfying these relationships proportionally in both the physical and trial space gives rise to the law of finite similitude, although it is revealed that coordinate invariant forms of vector and tensor products are required. Section 6 examines the view of the external observer, where a problem with a non-physical metric is revealed. It is shown in Section 7 how approximate forms of physically meaningful scaled experimentation can be obtained. Practical considerations and numerical investigations are provided in Sections 8 and 9 to showcase the range of applicability of the new theory.

\section{SPACE DISTORTION AND COORDINATE FRAMES}

Finite similitude is founded on the concept of space scaling, where a laboratory, a piece of equipment, a process or a specimen can be scaled by the contraction or expansion of space, which is termed here "space distortion". Evidently space distortion is practically impossible 
but what is possible is the examination of space distortion on processes and their comparison with scaled experimentation.

The starting point in the application of Newtonian physics is the stipulation of an inertial coordinate system. In scaling theory two are involved with coordinate systems $\boldsymbol{s}$ and $\boldsymbol{x}$ representing coordinate points in the physical and trial spaces respectively, where the full size and scaled processes reside. In line with Newtonian physics absolute times $t$ and $\tau$ are defined for each space. With these definitions scaling can be characterised by the two maps $d x=F \cdot d s$ and $d \tau=g d t$, where $t$ and $\tau$ pertain to the physical and trial spaces and both $F$ and $g$ are assumed to be both spatially and temporally invariant. Here $\boldsymbol{d x}=F \cdot \boldsymbol{d} \boldsymbol{s}$ can be viewed as an expression that simply relates differential coordinate components in the form $d x^{i}=F_{j}^{i}{ }_{j} s^{j}$, with no explicit reference to the underlying inertial frames. This is the approach adopted in reference [24], where isotropic scaling is discussed (i.e. $F=\beta I$ or $F^{i}{ }_{j}=\beta \delta_{j}^{i}$ ) with the implicit assumption that orthonormal inertial frames apply.

Two orthogonal systems are assumed to exist, one for the physical space, which houses the large-scale process and the other for the trial space where the scaled experiment resides. Consequently, it is equally viable to specify explicitly the orthonormal frames $\left\{\underline{G}_{i}\right\}$ and $\left\{\underline{g}_{i}\right\}$ for the physical and trial spaces, respectively as depicted in Fig. 1. In this case, vector representations for $\underline{d s}$ and $\underline{d x}$ are possible and take the form $\underline{d s}=\underline{G}_{i} d s^{i}$ and $\underline{d x}=\underline{g}_{i} d x^{i}$, being related by $\underline{d x}=\underline{F} \cdot \underline{d s}$. This immediately infers that $\underline{F}$ is a second-order $(1,1)$-tensor with coefficients $F^{i}{ }_{j}$ and bases $\underline{g}_{i} \otimes \underline{G}^{j}$ so that $\underline{\underline{F}}=F^{i}{ }_{j} \underline{g}_{i} \otimes \underline{G}^{j}$, where $\underline{g}_{i} \cdot \underline{g}^{j}=\delta_{i}^{j}$ and additionally $\underline{G}_{i} \cdot \underline{G}^{j}=\delta_{i}^{j}$, where $\left\{\underline{G}^{i}\right\}$ and $\left\{\underline{g}^{i}\right\}$ are dual systems of $\left\{\underline{G}_{i}\right\}$ and $\left\{\underline{g}_{i}\right\}$, respectively. Here $\delta_{i}^{j}$ is the well-established Kronecker delta symbol and in the case of an orthonormal frame the dual system adds nothing particularly new being identical to the originating system.

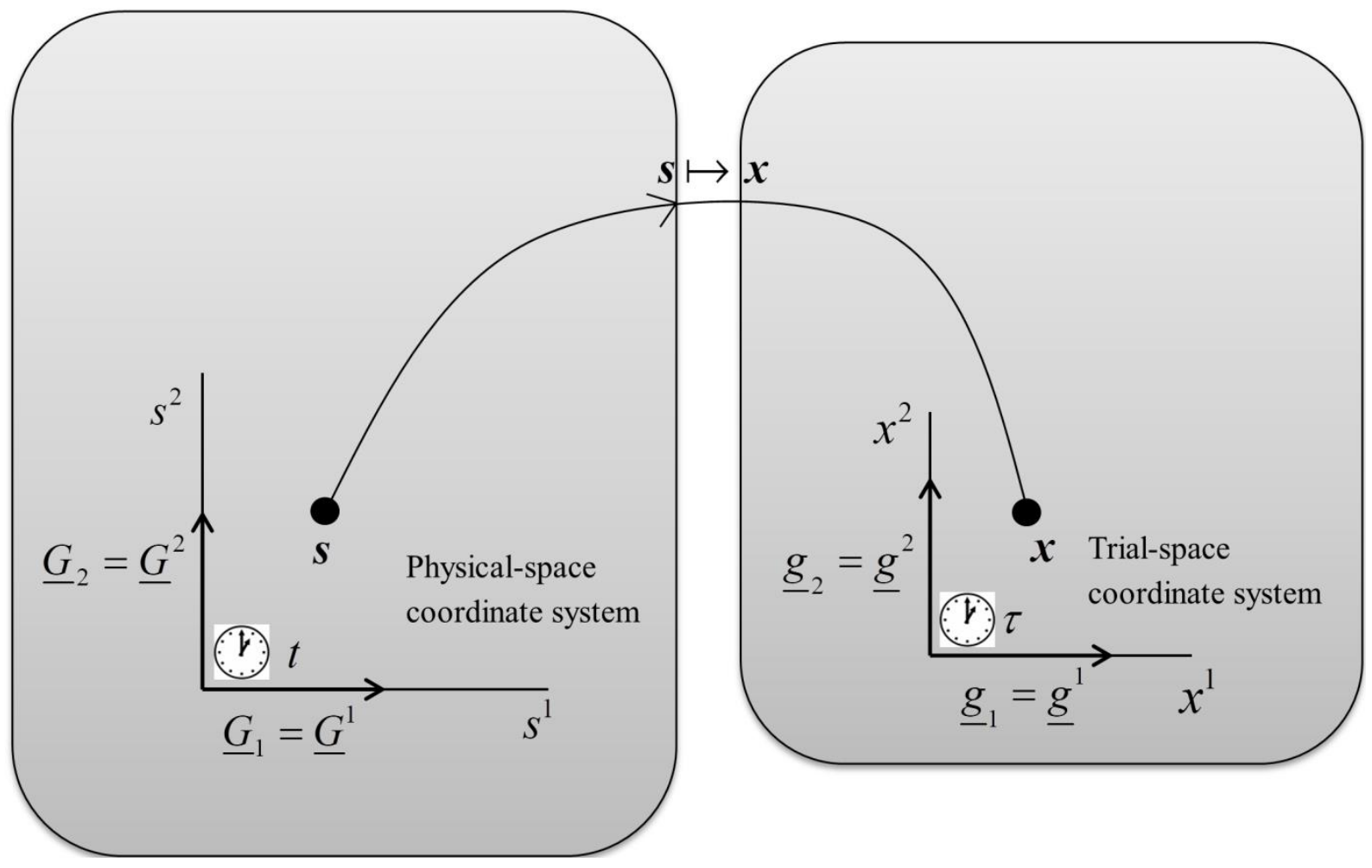


Fig. 1. Orthogonal coordinate systems in the physical and trial spaces

\subsection{Non-orthogonal Coordinate frames}

Dual frames are particularly useful with non-orthogonal frames and a particularly pertinent frame in space scaling is one that is skewed to match the consequences of the anisotropic distortion of space. It is important to appreciate that (with scaling) observation of the physics is from the viewpoint of an external observer who has the freedom to choose an appropriate coordinate frame. This point of view reflects the requirement in scaled experiments for the observer to be unaffected by the scaling process. Consider then the existence of a coordinate system in the trial space, which makes coordinate skewing invisible to an internal observer but not isotropic scaling (i.e. the observer has one foot in the trial space and one foot out). Note first that Nanson's first identity, which relate volumes in the two space is of the form $d V_{t s}=|F| d V_{p s}$ and it is useful at this point to set $J=|F|$ and $\beta=J^{1 / D}$, where $D$ is the topological dimension (typically $D=3$ ).

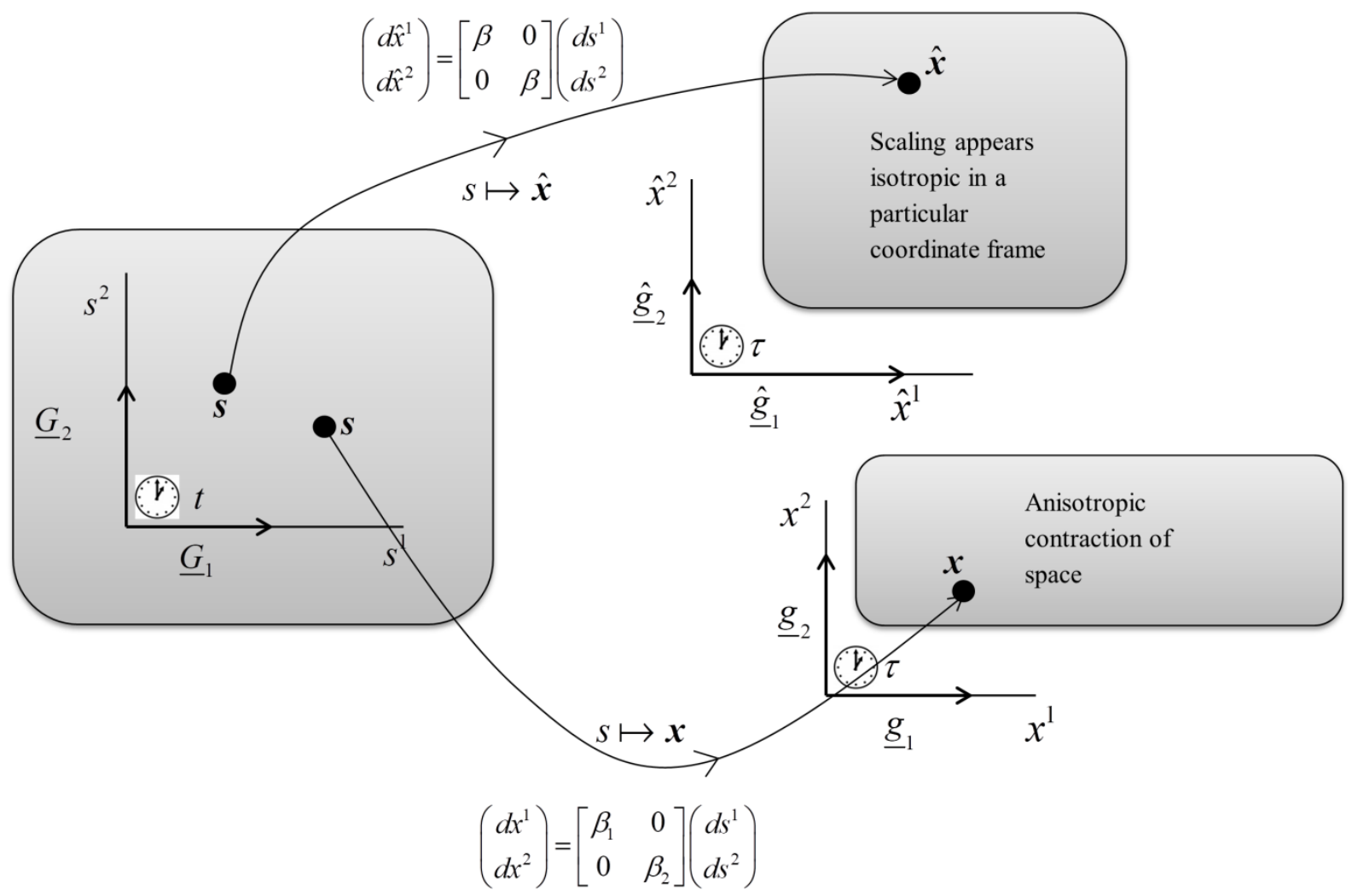

Fig. 2. Anisotropic space contraction (in 2D) as viewed in two coordinate-frames

The transformation that achieves this objective is $\boldsymbol{d} \hat{\boldsymbol{x}}=\beta F^{-1} \cdot \boldsymbol{d} \boldsymbol{x}$, which on substitution of $\boldsymbol{d} \boldsymbol{x}=F \cdot \boldsymbol{d} \boldsymbol{s}$ gives $\boldsymbol{d} \hat{\boldsymbol{x}}=\beta F^{-1} F \cdot \boldsymbol{d} \boldsymbol{s}=\beta \boldsymbol{d} \boldsymbol{s}$. A required property of a coordinate transformation (i.e. $\left.\boldsymbol{d} \hat{\boldsymbol{x}}=\beta F^{-1} \cdot \boldsymbol{d} \boldsymbol{x}\right)$ is vector invariance which infers that the vector differential $\underline{d \hat{x}}=\underline{\hat{g}}_{i} d \hat{x}^{i}$ must equal $\underline{d x}=\underline{g}_{i} d x^{i}$, i.e. $\underline{\hat{g}}_{i} d \hat{x}^{i}=\underline{g}_{i} d x^{i}$ but $\boldsymbol{d x}=\beta^{-1} F \cdot \boldsymbol{d} \hat{\boldsymbol{x}}$ or equivalently $d x^{i}=\beta^{-1} F^{i}{ }_{j} d \hat{x}^{j}$ so $\underline{\hat{g}}_{i} d \hat{x}^{i}=\underline{g}_{j} d x^{j}=\underline{g}_{j} \beta^{-1} F^{j}{ }_{i} d \hat{x}^{i}$ and consequently $\underline{\hat{g}}_{i}=\underline{g}_{j} \beta^{-1} F_{i}^{j} . \quad$ This basis has an associated dual defined to ensure $\underline{\hat{g}}_{i} \cdot \underline{\hat{g}}^{j}=\delta_{i}^{j}$ and is readily confirmed to be $\underline{\hat{g}}^{i}=\underline{g}^{j} \beta\left(F^{-1}\right)_{j}^{i}$ with the frames under discussion presented in Fig. 2 (in a 2-D frame). Note also that the 
tensor $\underline{\underline{F}}=F^{i}{ }_{j} \underline{g}_{i} \otimes \underline{G}^{j}$ can be represented with the new bases $\left\{\underline{\hat{g}}_{i}\right\}$ by substitution of $\underline{g}_{i}=\beta \underline{\hat{g}}_{j}\left(F^{-1}\right)_{i}^{j}$ to give

$\underline{\underline{\hat{F}}}=\beta\left(F^{-1}\right)_{i}^{j} F_{k}^{i} \underline{\hat{g}}_{j} \otimes \underline{G}^{k}=\beta \delta_{k}^{j} \underline{\hat{g}}_{j} \otimes \underline{G}^{k}=\beta \underline{\hat{I}}$

which confirms the vector identity $\underline{d \hat{x}}=\underline{\underline{F}} \cdot \underline{d s}=\beta \underline{\underline{I}} \cdot \underline{d s}$ and $\boldsymbol{d} \hat{\boldsymbol{x}}=\hat{F} \cdot \boldsymbol{d} \boldsymbol{s}=\beta \boldsymbol{d} \boldsymbol{s}$, where the observer with one-foot-in and one-foot-out of the trial space sees only isotropic scaling.

Application of Nanson's identity to $\boldsymbol{d} \hat{\boldsymbol{x}}=\hat{F} \cdot \boldsymbol{d} \boldsymbol{s}=\beta \boldsymbol{d} \boldsymbol{s}$ provides $d \hat{V}_{t s}=\beta^{D} d V_{p s}$ and contrasting with $d V_{t s}=\beta^{D} d V_{p s}$ confirms that $d \hat{V}_{t s}=d V_{t s}$, which is expected for a coordinate transformation. More about this can be found in Appendix A along with the derivation of the metric associated with the hat "^" coordinate system. The advantage of using this system in anisotropic scaling is that it provides a convenient route to the important scaling identities since anisotropic scaling is equivalent to isotropic scaling (i.e. $\boldsymbol{d} \hat{\boldsymbol{x}}=\boldsymbol{\beta} \boldsymbol{d} \boldsymbol{s}$ ) and a coordinate transformation.

\section{THE LAW OF VOLUME}

The ability to describe physics on a finite volume is achievable with the use of transport equations which are founded on control volume concepts. Thus, the starting point to the application of integral continuum transport forms is a mathematical theory underpinning the movement of a control volume $\Omega_{p s}^{*}$ in the physical space transported by means of the velocity field $\underline{v}_{p s}^{*}$. A control volume is defined to be a continuous open domain $\Omega_{p s}^{*}$ whose closure contains the boundary $\Gamma_{p s}^{*}$, which possesses on outward pointing unit normal $\underline{n}_{p s}^{*}$ and, which satisfies the law of volume

$$
\frac{D^{*}}{D^{*} t} \int_{\Omega_{p s}^{*}} d V_{p s}^{*}=\int_{\Gamma_{p s}^{*}} \underline{v}_{p s}^{*} \cdot \underline{n}_{p s}^{*} d \Gamma_{p s}^{*}=\int_{\Gamma_{p s}^{*}} \boldsymbol{v}_{p s}^{*} \cdot \boldsymbol{n}_{p s}^{*} d \Gamma_{p s}^{*}
$$

where movement of $\Omega_{p s}^{*}$ is described by a diffeomorphism (invertible differential map) $\boldsymbol{s}^{*}\left(\chi_{p s}, t\right): \Omega_{p s}^{* r e f} \rightarrow \Omega_{p s}^{*}$, and where $\Omega_{p s}^{* r e f}$ is a continuous reference space for the control volume $\Omega_{p s}^{*}$ and $\chi_{p s}$ is a coordinate function in this space. 


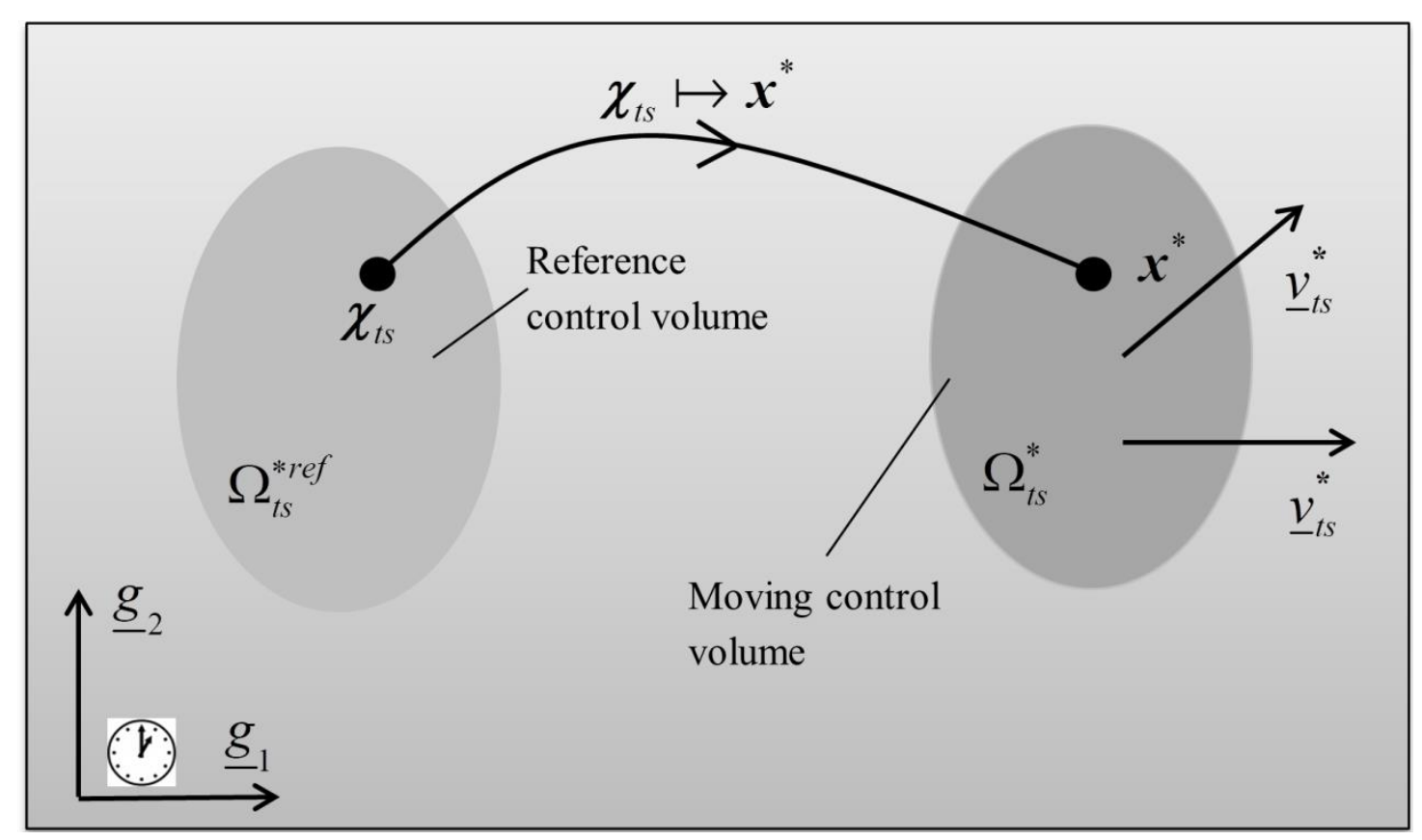

Fig. 3. Reference and moving control volumes in the trial space

Note that the identity $\underline{v}_{p s}^{*} \cdot \underline{n}_{p s}^{*}=\boldsymbol{v}_{p s}^{*} \cdot \boldsymbol{n}_{p s}^{*}$ applied in Eq. (2) signifies that the inner product can be viewed in two ways, i.e. not only as a product between vectors but as an algebraic identity as well. The use of the derivative $D^{*} / D^{*} t$ in Eq. (2) (rather than the ordinary derivative $d / d t$ ) is intended to immediately relay the notion that $\Omega_{p s}^{*}$ is a control volume transported by means of the velocity $v_{p s}^{*}$. The same apparatus can be invoked to apply to the trial space in which the scaled domain $\Omega_{t s}$ resides (see Fig. 3). Allowance is made for the possibility that time in the trial space can run at a different rate with use of the symbol $\tau$ as opposed to $t$. The map $\boldsymbol{x}^{*}\left(\chi_{t s}, \tau\right)$ gives rise to the law of volume

$$
\frac{D^{*}}{D^{*} \tau} \int_{\Omega_{t s}^{*}} d V_{t s}^{*}=\int_{\Gamma_{t s}^{*}} \boldsymbol{v}_{t s}^{*} \cdot \boldsymbol{n}_{t s}^{*} d \Gamma_{t s}^{*}=\int_{\Gamma_{t s}^{*}} \hat{\boldsymbol{v}}_{t s}^{*} \cdot \hat{\boldsymbol{n}}_{t s}^{*} d \Gamma_{t s}^{*}
$$

where $\hat{\boldsymbol{v}}_{t s}^{*}$ is simply $\boldsymbol{v}_{t s}^{*}$ but defined in the coordinate frame defined in Section 2 and depicted in Fig. 2, and it is assumed further that $\hat{\boldsymbol{v}}_{t s}^{*} \cdot \hat{\boldsymbol{n}}_{t s}^{*}=\boldsymbol{v}_{t s}^{*} \cdot \boldsymbol{n}_{t s}^{*}\left(\right.$ or $\left.\underline{\hat{v}}_{t s}^{*} \cdot \underline{\hat{n}}_{t s}^{*}=\underline{v}_{t s}^{*} \cdot \underline{n}_{t s}^{*}\right)$ since the inner product is independent of the coordinate system.

\section{Theorem 3.1: Proportional laws of Volume}

Eq. (2) is proportional to Eq. (3) (for arbitrary $\Omega_{p s}^{*}$ ) if and only if $\boldsymbol{v}_{p s}^{*}=g F^{-1} \cdot v_{t s}^{*}$ where the differential identities $d \tau=g d t$ and $\boldsymbol{d} \boldsymbol{x}^{*}=F \cdot \boldsymbol{d} \boldsymbol{s}^{*}$ (and $\boldsymbol{d} \boldsymbol{\chi}_{t s}=F \cdot \underline{\boldsymbol{d}}_{p s}$ ) apply, and where $F$ is the invariant scaling map introduced in Section 2.

\section{Proof 3.1: Proportional laws of Volume}

The differential $\boldsymbol{d} \boldsymbol{x}^{*}=F_{t s} \cdot \boldsymbol{d} \chi_{t s}+v_{t s}^{*} d \tau$ arising from the map $\boldsymbol{x}^{*}\left(\chi_{t s}, \tau\right): \Omega_{t s}^{* \text { ref }} \mapsto \Omega_{t s}^{*}$ is reproduced from the differentials (and associated maps) $\boldsymbol{d} \boldsymbol{x}^{*}=F \cdot \boldsymbol{d} \boldsymbol{s}^{*}, \boldsymbol{d} \chi_{p s}=F^{-1} \cdot \boldsymbol{d} \chi_{t s}$ and 
$\boldsymbol{d} \boldsymbol{s}^{*}=F_{p s} \cdot \boldsymbol{d} \chi_{p s}+\boldsymbol{v}_{p s}^{*} d t$ since substitution provides $\boldsymbol{d} \boldsymbol{x}^{*}=F F_{p s} F^{-1} \cdot \boldsymbol{d} \chi_{t s}+g^{-1} F \cdot v_{p s}^{*} d \tau$ and matching is achieved on setting $v_{p s}^{*}=g F^{-1} \cdot v_{t s}^{*}$ and $F_{t s}=F F_{p s} F^{-1}$. It immediately follows from Nanson's first identity that $d V_{t s}^{*}=\left|F_{t s}\right| d V_{t s}^{* r e f}=J\left|F_{p s}\right| d V_{p s}^{* r e f}=J d V_{p s}^{*}$, which on substitution into Eq. (3) along with $v_{p s}^{*}=g F^{-1} \cdot v_{t s}^{*}$ gives

$$
\frac{D^{*}}{D^{*} \tau} \int_{\Omega_{s s}^{*}} d V_{t s}^{*}=\frac{J}{g} \frac{D^{*}}{D^{*} t} \int_{\Omega_{p s}^{*}} d V_{p s}^{*}=\frac{1}{g} \int_{\Gamma_{t s}^{*}} \boldsymbol{v}_{p s}^{*} \cdot F^{-T} \cdot \boldsymbol{n}_{t s}^{*} d \Gamma_{t s}^{*}=\frac{J}{g} \int_{\Gamma_{p s}^{*}} \boldsymbol{v}_{p s}^{*} \cdot \boldsymbol{n}_{p s}^{*} d \Gamma_{p s}^{*}
$$

which is evidently proportional to Eq. (3) and where Nanson's second identity is applied to arrive at the integral on the far right hand side of Eq. (4).

In summary Theorem 3.1 provides the following identity

$$
\left(\frac{D^{*}}{D^{*} \tau} \int_{\Omega_{t s}^{*}} d V_{t s}^{*}=\int_{\Gamma_{t s}^{*}} \hat{\boldsymbol{v}}_{t s}^{*} \cdot \hat{\boldsymbol{n}}_{t s}^{*} d \Gamma_{t s}^{*}\right)=\frac{J}{g}\left(\frac{D^{*}}{D^{*} t} \int_{\Omega_{p s}^{*}} d V_{p s}^{*}-\int_{\Gamma_{p s}^{*}} \boldsymbol{v}_{p s}^{*} \cdot \boldsymbol{n}_{p s}^{*} d \Gamma_{p s}^{*}\right)
$$

which encapsulates the finite similitude approach to anisotropic scaling by confirming that the solution to an equation in one space corresponds to the solution in another and vice versa.

Note also the simplification achieved with the hat " $\wedge$ " coordinate system as it provides proportional relationships of the type $\boldsymbol{v}_{p s}^{*}=g \beta^{-1} \hat{\boldsymbol{v}}_{t s}^{*}$. Since physics must be independent of the coordinate system any system can be selected but some choices are more convenient and for anisotropic scaling the hat system is particularly convenient as it results in scaling identities for isotropic scaling [24] as is confirmed in the following section.

\section{GENERIC SCALING IDENTITIES}

Finite similitude is founded on the metaphysical process of space distortion which necessitates physical formulations that reflect this aspect. The natural choice is a controlvolume formulation since control volumes are simply regions of space so any change in the background space is immediately reflected in the control volume. Transport equations in integral form provide the appropriate description for both conservation and non-conservation laws of nature and describe the behaviour of field variable $\psi_{p s}$ defined on a moving control volume $\Omega_{p s}^{*}$ by

$$
\frac{D^{*}}{D^{*} t} \int_{\Omega_{p s}^{*}} \rho_{p s} \psi_{p s} d V_{p s}^{*}+\int_{\Gamma_{p s}^{*}} \rho_{p s} \psi_{p s}\left(\boldsymbol{v}_{p s}-\boldsymbol{v}_{p s}^{*}\right) \cdot \boldsymbol{n}_{p s}^{*} d \Gamma_{p s}^{*}=-\int_{\Gamma_{p s}^{*}} \boldsymbol{J}_{p s}^{\psi} \cdot \boldsymbol{n}_{p s}^{*} d \Gamma_{p s}^{*}+\int_{\Omega_{p s}^{*}} \rho_{p s} \boldsymbol{b}_{p s}^{\psi} d V_{p s}^{*}
$$

where $\rho_{p s}$ is mass density, $\boldsymbol{v}_{p s}$ is material velocity, $\boldsymbol{J}_{p s}^{\psi}$ is a flux term and $\boldsymbol{b}_{p s}^{\psi}$ is a source term.

It is important to appreciate that the law of volume is built into Eq. (6) and explains the presence of the control volume velocity $\boldsymbol{v}_{p s}^{*}$. In order to invoke similarity concepts it is necessary to consider the existence of a transport equation identical in form on the trial space. Consider then the movement of control volume $\Omega_{t s}^{*}$ transported with velocity $v_{t s}^{*}$ and the associated transport equation 


$$
\frac{D^{*}}{D^{*} \tau} \int_{\Omega_{t s}^{*}} \rho_{t s} \psi_{t s} d V_{t s}^{*}+\int_{\Gamma_{t s}} \rho_{t s} \psi_{t s}\left(\boldsymbol{v}_{t s}-\boldsymbol{v}_{t s}^{*}\right) \cdot \boldsymbol{n}_{t s}^{*} d \Gamma_{t s}^{*}=-\int_{\Gamma_{t s}^{*}} \boldsymbol{J}_{t s}^{\psi} \cdot \boldsymbol{n}_{t s}^{*} d \Gamma_{t s}^{*}+\int_{\Omega_{t s}^{*}} \rho_{t s} \boldsymbol{b}_{t s}^{\psi} d V_{t s}^{*}
$$

where similarly the control volume $\Omega_{t s}$ transported with velocity $\boldsymbol{v}_{t s}^{*}$.

Eq. (7) is defined on the orthonormal frame depicted in Fig. 2 but the hat "^" system can also be used and takes the form

$$
\frac{D^{*}}{D^{*} \tau} \int_{\Omega_{t s}^{*}} \rho_{t s} \hat{\psi}_{t s} d V_{t s}^{*}+\int_{\Gamma_{t s}} \rho_{t s} \hat{\psi}_{t s}\left(\hat{\boldsymbol{v}}_{t s}-\hat{\boldsymbol{v}}_{t s}^{*}\right) \cdot \hat{\boldsymbol{n}}_{t s}^{*} d \Gamma_{t s}^{*}=-\int_{\Gamma_{t s}^{*}} \hat{\boldsymbol{J}}_{t s}^{\psi} \cdot \hat{\boldsymbol{n}}_{t s}^{*} d \Gamma_{t s}^{*}+\int_{\Omega_{t s}^{*}} \rho_{t s} \hat{\boldsymbol{b}}_{t s}^{\psi} d V_{t s}^{*}
$$

which captures the same physics as Eq. (7) but is in a form that is best suited to anisotropic scaling.

Eqs. (6) and (8) are presently unrelated but Theorem 3.1 highlights how these can be related by synchronising the control volume motion with a map $\boldsymbol{s}^{*} \mapsto \hat{\boldsymbol{x}}^{*}$ and an associated differential identity $\boldsymbol{d} \hat{\boldsymbol{x}}^{*}=\beta \boldsymbol{d} \boldsymbol{s}^{*}$, which immediately gives rise to Nanson's identities $d V_{t s}^{*}=\beta^{3} d V_{p s}^{*}$ and $\boldsymbol{d} \hat{\Gamma}_{t s}^{*}=\beta^{2} d \Gamma_{p s}^{*}$, where $d V_{t s}^{*}$ is an elemental volume in $\Omega_{t s}^{*}$ and $\boldsymbol{d} \hat{\Gamma}_{t s}^{*}=\hat{\boldsymbol{n}}_{t s}^{*} d \Gamma_{t s}^{*}$ with $\hat{\boldsymbol{n}}_{t s}^{*}$ being an outward pointing unit normal on $\Gamma_{t s}^{*}$ the boundary of $\Omega_{t s}^{*}$. Substitution of these identities into Eq. (8) along with multiplication by scalar $\alpha^{\psi}$ (a constant of proportionality) and $g$ (since $d \tau=g d t$ ) gives rise to the transport equation

$$
\begin{aligned}
\frac{D^{*}}{D^{*} t} \int_{\Omega_{p s}^{*}} \alpha^{\psi} \rho_{t s} \beta^{3} \hat{\psi}_{t s} d V_{p s}^{*} & +\int_{\Gamma_{p s}^{*}} \alpha^{\psi} \rho_{t s} \beta^{3} \hat{\psi}_{t s}\left[g \beta^{-1}\left(\hat{\boldsymbol{v}}_{t s}-\hat{\boldsymbol{v}}_{t s}^{*}\right)\right] \cdot \boldsymbol{n}_{p s}^{*} d \Gamma_{p s}^{*}= \\
& -\int_{\Gamma_{p s}^{*}} \alpha^{\psi} g \beta^{2} \hat{\boldsymbol{J}}_{t s}^{\psi} \cdot \boldsymbol{n}_{p s}^{*} d \Gamma_{p s}^{*}+\int_{\Omega_{p s}^{*}} g \alpha^{\psi} \rho_{p s} \beta^{3} \hat{\boldsymbol{b}}_{t s}^{\psi} d V_{p s}^{*}
\end{aligned}
$$

which is either scalar or vector depending on whether $\hat{\psi}_{t s}$ is scalar or vector.

It is evident on examination of Eqs. (6) and (9) that the physics is proportional on an arbitrary control volume if and only if the corresponding integrands in the transport equations match, i.e.

$$
\begin{aligned}
& \rho_{p s} \psi_{p s}=\alpha^{\psi} \rho_{t s} \beta^{3} \hat{\psi}_{t s} \\
& \rho_{p s} \psi_{p s}\left(\boldsymbol{v}_{p s}-\boldsymbol{v}_{p s}^{*}\right)=\alpha^{\psi} \rho_{t s} \beta^{3} \hat{\psi}_{t s} g \beta^{-1}\left(\hat{\boldsymbol{v}}_{t s}-\hat{\boldsymbol{v}}_{t s}^{*}\right) \\
& \boldsymbol{J}_{p s}^{\psi}=\alpha^{\psi} g \beta^{2} \hat{\boldsymbol{J}}_{t s}^{\psi} \\
& \rho_{p s} \boldsymbol{b}_{p s}^{\psi}=g \alpha^{\psi} \rho_{t s} \beta^{3} \hat{\boldsymbol{b}}_{t s}^{\psi}
\end{aligned}
$$

where it is understood that Eqs. (10b) and (10c) arise out of the arbitrariness of the direction of outward pointing normal $\boldsymbol{n}_{p s}^{*}$ and the fact that the dot product is nondegenerate.

Eqs. (10) provides the central conditions for similitude although does not necessarily confirm that such a solution exists. Note also that Eq. (10a) can be substituted into Eq. (10b), which is then automatically satisfied by $\boldsymbol{v}_{p s}^{*}=g \beta^{-1} \hat{\boldsymbol{v}}_{t s}^{*}$ (see Section 3) and the material relationship 
$\boldsymbol{v}_{p s}=g \beta^{-1} \hat{\boldsymbol{v}}_{t s}$

where it is understood that this along with Eqs. (10) are coefficient identities and not vector identities.

\section{PARTICULAR SCALING IDENTITIES}

In order to establish that similitude exists and that Eqs. (10) can be realised it is necessary to consider each transport equation in turn. The transport equations of interest in this paper are equations for volume, continuity, momentum, movement, energy and entropy, which are

$$
\begin{aligned}
& \frac{D^{*}}{D^{*} t} \int_{\Omega_{p s}^{*}} d V_{p s}^{*}-\int_{\Gamma_{p s}^{*}} \boldsymbol{v}_{p s}^{*} \cdot \boldsymbol{n}_{p s} d \Gamma_{p s}^{*}=0 \\
& \frac{D^{*}}{D^{*} t} \int_{\Omega_{p s}^{*}} \rho_{p s} d V_{p s}^{*}+\int_{\Gamma_{p s}^{*}} \rho_{p s}\left(\boldsymbol{v}_{p s}-\boldsymbol{v}_{p s}^{*}\right) \cdot \boldsymbol{n}_{p s} d \Gamma_{p s}^{*}=0 \\
& \frac{D^{*}}{D^{*} t} \int_{\Omega_{p s}^{*}} \rho_{p s} \boldsymbol{v}_{p s} d V_{p s}^{*}+\int_{\Gamma_{p s}^{*}} \rho_{p s} \boldsymbol{v}_{p s}\left(\boldsymbol{v}_{p s}-\boldsymbol{v}_{p s}^{*}\right) \cdot \boldsymbol{n}_{p s} d \Gamma_{p s}^{*}=\int_{\Gamma_{p s}^{*}} \boldsymbol{\sigma}_{p s} \cdot \boldsymbol{n}_{p s} d \Gamma_{p s}^{*}+\int_{\Omega_{p s}^{*}} \rho_{p s} \boldsymbol{b}_{p s} d V_{p s}^{*} \\
& \frac{D^{*}}{D^{*} t} \int_{\Omega_{p s}^{*}} \rho_{p s} \boldsymbol{u}_{p s} d V_{p s}^{*}+\int_{\Gamma_{p s}^{*}} \rho_{p s} \boldsymbol{u}_{p s}\left(\boldsymbol{v}_{p s}-\boldsymbol{v}_{p s}^{*}\right) \cdot \boldsymbol{n}_{p s} d \Gamma_{p s}^{*}=\int_{\Omega_{p s}^{*}} \rho_{p s} \boldsymbol{v}_{p s} d V_{p s}^{*} \\
& \frac{D^{*}}{D^{*} t} \int_{\Omega_{p s}^{*}} \rho_{p s} e_{p s} d V_{p s}^{*}+\int_{\Gamma_{p s}^{*}} \rho_{p s} e_{p s}\left(\boldsymbol{v}_{p s}-\boldsymbol{v}_{p s}^{*}\right) \cdot \boldsymbol{n}_{p s} d \Gamma_{p s}^{*}= \\
& \int_{\Gamma_{p s}^{*}} \boldsymbol{v}_{p s} \cdot \boldsymbol{\sigma}_{p s} \cdot \boldsymbol{n}_{p s} d \Gamma_{p s}^{*}-\int_{\Gamma_{p s}^{*}} \boldsymbol{q}_{p s} \cdot \boldsymbol{n}_{p s} d \Gamma_{p s}^{*}+\int_{\Omega_{p s}^{*}} \rho_{p s} Q_{p s} d V_{p s}^{*}+\int_{\Omega_{p s}^{*}} \rho_{p s} \boldsymbol{v}_{p s} \cdot \boldsymbol{b}_{p s} d V_{p s}^{*} \\
& \frac{D^{*}}{D^{*} t} \int_{\Omega_{p s}^{*}} \rho_{p s} s_{p s} d V_{p s}^{*}+\int_{\Gamma_{p s}^{*}} \rho_{p s} s_{p s}\left(\boldsymbol{v}_{p s}-\boldsymbol{v}_{p s}^{*}\right) \cdot \boldsymbol{n}_{p s} d \Gamma_{p s}^{*}= \\
& \quad-\int_{\Gamma_{p s}^{*}} T_{p s}^{-1} \boldsymbol{q}_{p s} \cdot \boldsymbol{n}_{p s} d \Gamma_{p s}^{*}+\int_{\Omega_{p s}^{*}} \rho_{p s} T_{p s}^{-1} Q_{p s} d V_{p s}^{*}+\int_{\Omega_{p s}^{*}} \dot{s}_{p s} d V_{p s}^{*}
\end{aligned}
$$

where $e_{p s}=u_{p s}+\frac{1}{2} \boldsymbol{v}_{p s} \cdot \boldsymbol{v}_{p s}, u_{p s}$ is stationary specific internal energy, and $s_{p s}$ is specific entropy, $\boldsymbol{q}_{p s} \cdot \boldsymbol{n}_{p s}$ is heat flux, $Q_{p s}$ represents a heat source, $\boldsymbol{\sigma}_{p s}$ is the Cauchy stress tensor, $\boldsymbol{b}_{p s}$ is a body force and $\dot{s}_{p s} \geq 0$ is an entropy density production term associated with irreversibility.

It is apparent that $\psi$ can be set to be either $\rho^{-1}, 1, v, u, e$, or $s$ in Eq. (6) to arrive at transport equations for the physical and trial spaces. It is important to note that although the transport equations include transfers at boundaries of domains they do not involve constitutive equations. This feature is critical to the theory as finite similitude does not depend on constitutive laws. It should be mentioned though that with anisotropic scaling, constitutive laws can impose unrealistic behaviour on the scaled materials, which is a feature absent in the case of isotropic scaling (see refs [24-28]). Finite similitude is defined here to be the matching of transport Eq. (6) with Eq. (9) for Eqs. (12) on a finite domain. This leads to the following identities for isotropic scaling derived in reference [24]: 
scaling parameters: $\left\{\alpha^{1}=\beta^{-3}, \alpha^{v}=g \beta^{-1} \alpha^{\rho}, \alpha^{u}=\beta^{-1} \alpha^{\rho}, \alpha^{e}=g^{2} \beta^{-2} \alpha^{\rho}\right\}$;

field variables: $\left\{\rho_{p s}=\alpha^{\rho} \beta^{3} \rho_{t s}, v_{p s}=g \beta^{-1} \hat{\boldsymbol{v}}_{t s}, \boldsymbol{u}_{p s}=\beta^{-1} \hat{\boldsymbol{u}}_{t s}, u_{p s}=g^{2} \beta^{-2} u_{t s}, \alpha^{s} T_{p s}=\alpha^{e} T_{t s}\right\}$;

transfer identities: $\left\{\boldsymbol{\sigma}_{p s}=\alpha^{v} g \beta^{2} \hat{\boldsymbol{\sigma}}_{t s}, \boldsymbol{q}_{p s}=\alpha^{e} g \beta^{2} \hat{\boldsymbol{q}}_{t s}\right\}$ and;

source identities: $\left\{\rho_{p s} Q_{p s}=\alpha^{e} g \beta^{3} \rho_{t s} Q_{t s}, \rho_{p s} \boldsymbol{b}_{p s}=\alpha^{v} g \beta^{3} \rho_{t s} \hat{\boldsymbol{b}}_{t s}, \dot{s}_{p s}=\alpha^{s} g \beta^{3} \dot{s}_{t s}\right\}$.

which confirms that finite similitude exists with anisotropic scaling as viewed by an observer that is able to view isotropic scaling only.

\section{Theorem 5.1: Law of Finite Similitude for Anisotropic Scaling}

Two finite domains have finite similitude (i.e. Eqs (6) and (9)) are proportional for Eqs (12)) if and only if their time scales are linearly related and their coordinate spaces are related by an affine transformation and frame-invariant (metric-free) vector and tensor products are adopted.

\section{Proof: Law of Finite Similitude for Anisotropic Scaling}

A feature of the formal proof requires all of the transport equations above to be considered and scaling identities for each obtained (see reference [24] for details). Unlike the identities obtained in reference [24] however the ones above differ in that the coordinate frame in the trial space is not orthonormal. This has no impact on scalar identities but in the case of vector and tensor identities it has an impact, since these are required to be returned to the orthonormal frame in the trial space as discussed in the next section.

\section{THE PROBLEM WITH THE METRIC}

In order to apply the analysis is Section 5 to an anisotropically scaled experiment it is first necessary to return expressions in the frame $\left\{\hat{g}_{i}\right\}$ to $\left\{g_{i}=G_{i}\right\}$, i.e. to the viewpoint of an external observer. This is relatively straightforward as it requires only the application of the appropriate coordinate transformation (see Section 2). Scalars are unaffected by this transformation but vector $\hat{\underline{v}}_{t s}=\hat{v}_{t s}^{i} \hat{g}_{i}$ and tensor $\hat{\hat{\sigma}}_{t s}=\hat{\sigma}_{t s}^{i j} \hat{g}_{i} \otimes \hat{g}_{j}$ (say) transform on substitution of $\underline{g}_{i}=\beta \underline{\hat{g}}_{j}\left(F^{-1}\right)_{i}^{j}$ to

$\underline{v}_{t s}=v_{t s}^{j} g_{j}=\underline{\hat{v}}_{t s}=\hat{v}_{t s}^{i} \hat{g}_{i}=\beta^{-1} F^{j}{ }_{i} \hat{v}_{t s}^{i} \underline{g}_{j}$

and

$\underline{\underline{\sigma}}_{t s}=\sigma_{t s}^{k m} g_{k} \otimes g_{m}=\underline{\underline{\sigma}}_{t s}=\hat{\sigma}_{t s}^{i j} \hat{g}_{i} \otimes \hat{g}_{j}=\beta^{-1} F^{k}{ }_{i} \hat{\sigma}_{t s}^{i j} \beta^{-1} F^{m}{ }_{j} \hat{g}_{k} \otimes \hat{g}_{m}$

which provides $v_{t s}^{i}=\beta^{-1} F^{i}{ }_{j} \hat{v}_{t s}^{j} \quad\left(\right.$ or $\left.\quad \boldsymbol{v}_{t s}=\beta^{-1} F \cdot \hat{\boldsymbol{v}}_{t s}\right)$ and $\sigma_{t s}^{i j}=\beta^{-1} F^{i}{ }_{k} \hat{\sigma}_{t s}^{k m} \beta^{-1} F^{j}{ }_{m} \quad$ (or $\left.\sigma_{t s}=\beta^{-2} F \cdot \hat{\sigma}_{t s} \cdot F^{T}\right)$.

Similarly, on substitution of $\underline{\hat{g}}^{i}=\underline{g}^{j} \beta\left(F^{-1}\right)_{j}^{i}$ into

$\underline{v}_{t s}=v_{t s j} g^{j}=\hat{v}_{t s}=\hat{v}_{t s i} \hat{g}^{i}=\beta\left(F^{-1}\right)_{j}^{i} \hat{v}_{t s i} \underline{g}^{j}$ 
gives $v_{t s i}=\beta\left(F^{-1}\right)_{i}^{j} \hat{v}_{t s j}$ (or $v_{t s}=\beta F^{-T} \cdot \hat{v}_{t s}$ ) where the appearance of the transpose indicates that covariant coefficients are being related.

Application of the transformation rules to some of the important identities in Section 5 leads to the relationships listed in Table 1. Although finite similitude requires no reference to constitutive equations these are nonetheless involved and the effect of the relationships presented in Table 1 can be found in Appendix B for linear materials.

Table 1. Scaling relationships for contra/co-variant components of physical fields

\begin{tabular}{|c|c|c|}
\hline Physical Field & $\begin{array}{c}\text { Scaling Rule for } \\
\text { Contravariant Components }\end{array}$ & $\begin{array}{c}\text { Scaling Rule for } \\
\text { Covariant Components }\end{array}$ \\
\hline$\rho_{p s}$ & $\rho_{p s}=\alpha^{\rho} \beta^{3} \rho_{t s}$ & $\rho_{p s}=\alpha^{\rho} \beta^{3} \rho_{t s}$ \\
\hline$T_{p s}$ & $\alpha^{s} T_{p s}=\alpha^{e} T_{t s}$ & $\alpha^{s} T_{p s}=\alpha^{e} T_{t s}$ \\
\hline$\underline{u}_{p s}=u_{p s}^{i} \underline{G}_{i}=u_{p s i} \underline{G}^{i}$ & $\boldsymbol{u}_{t s}=F \cdot \boldsymbol{u}_{p s}$ & $F^{T} \cdot \boldsymbol{u}_{t s}=\beta^{2} \boldsymbol{u}_{p s}$ \\
\hline$\underline{v}_{p s}=v_{p s}^{i} \underline{G}_{i}=v_{p s i} \underline{G}^{i}$ & $g \boldsymbol{v}_{t s}=F \cdot \boldsymbol{v}_{p s}$ & $g F^{T} \cdot \boldsymbol{v}_{t s}=\beta^{2} \boldsymbol{v}_{p s}$ \\
\hline$\underline{\sigma}_{p s}=\sigma_{p s}^{i j} \underline{G}_{i} \otimes \underline{G}_{j}=\sigma_{p s i j} \underline{G}^{i} \otimes \underline{G}^{j}$ & $g^{2} \rho_{t s}^{-1} \boldsymbol{\sigma}_{t s}=\rho_{p s}^{-1} F \boldsymbol{\sigma}_{p s} F^{T}$ & $g^{2} \rho_{t s}^{-1} \boldsymbol{\sigma}_{t s}=\rho_{p s}^{-1} \beta^{4} F^{-T} \boldsymbol{\sigma}_{p s} F^{-1}$ \\
\hline
\end{tabular}

Note that an issue with the metric arises with kinetic energy similarity and the identity $\boldsymbol{v}_{p s}=g F^{-1} \cdot \boldsymbol{v}_{t s}$, as this provides

$$
g^{2}\left(F^{-1} \cdot \boldsymbol{v}_{t s}\right) \cdot\left(F^{-1} \cdot \boldsymbol{v}_{t s}\right)=g^{2} \boldsymbol{v}_{t s} \cdot F^{-T} F^{-1} \cdot \boldsymbol{v}_{t s}=g^{2} \beta^{-2} \boldsymbol{v}_{t s} \cdot\left(\beta^{2} F^{-T} F^{-1}\right) \cdot \boldsymbol{v}_{t s}=\boldsymbol{v}_{p s} \cdot \boldsymbol{v}_{p s}=v_{p s}^{2}
$$

which confirms that $v_{p s}^{2}$ is not generally a scalar multiple of $v_{t s}^{2}$.

The essence of the problem is the appearance of the metric tensor $g_{n p}=\beta^{2} F^{-1} F^{-T}$ (or more precisely $\underset{\underline{g}}{g}=\beta^{2} \underline{\underline{F}}^{-1} \cdot \underline{F}^{-T}$ ), which is non-physical since the inertial coordinate systems are by definition orthonormal. The discussion in Appendix A reveals the source of the metric being associated with the skewing of any attached coordinate system as illustrated in Fig. 2 .

\section{PHYSICAL-FINITE SIMILITUDE}

With anisotropic scaling, finite similitude (i.e. proportional transport equations) has been shown to be possible on adopting a dual coordinate system. However, for the similitude to be physically realisable it is necessary to represent the trial-space equations in a form expected for the physical system under consideration. In the case of deformation an issue arises with the momentum equation (which despite passing the similitude test) involves contravariant velocity $v_{t s}^{i}$ but covariant displacement $u_{t s i}$ on adoption of the constitutive relationship $\sigma_{t s}^{i j}=C_{t s}^{i j k l} \varepsilon_{t s k l}$ (see Appendix B1). The raising or lowering of the coefficients introduces a non-physical aspect into the governing equations and must be avoided for physically meaningful finite similitude. One possibility (not considered here) is to allow the constitutive law $\underline{\underline{\sigma}}_{t s}=\underline{\underline{\underline{C}}}_{\mathrm{ts}}: \underline{\varepsilon}_{t s}$ to take on a non-standard form but this is ruled out here because of the rarity of materials satisfying such behaviour. Note that in the absence of velocity $v_{t s}^{i}$ (i.e. 
static problems) there is no requirement to raise the coefficient of displacement $u_{t s i}$ and physical-finite similitude is returned. Similarly in rigid-body dynamics, the absence of covariant displacement $u_{t s i}$ makes physical-finite similitude possible. There exists however another class of important problems that are physically realisable, which occur when one of the components of velocity (or displacement) is dominant.

\subsection{The approximated metric}

To examine this case it is assumed that the $3^{\text {rd }}$ component of $\underline{u}_{t s}$ (i.e. the z-component $u_{t s}^{3}$ ) is significantly larger than the other two components. The approximation adopted here is markedly simple but surprisingly useful as problems involving a dominant direction are manifold in engineering (e.g. plate vibration). In the case of plate distortion a simple modification to the constitutive equation arising from the raising of the index on displacement is needed. To show this recall that covariant and contravariant displacements satisfy $\boldsymbol{u}_{t s}=\beta^{2} F^{-T} \cdot \boldsymbol{u}_{p s}$ (or $\left.u_{t s i}=\beta^{2}\left(F^{-1}\right)^{j}{ }_{i} u_{p s j}\right)$ and $\boldsymbol{u}_{t s}=F \cdot \boldsymbol{u}_{p s}$ or $\left(u_{t s}^{i}=F^{i}{ }_{j} u_{p s}^{j}\right)$ (see Table 1), so it follows that the contravariant dot product gives $u_{p s}^{2}=\beta^{-2} \boldsymbol{u}_{t s} \cdot g_{n p} \cdot \boldsymbol{u}_{t s}$ and similarly for the covariant case $u_{p s}^{2}=\beta^{-2} \boldsymbol{u}_{t s} \cdot g_{n p}^{-1} \cdot \boldsymbol{u}_{t s}$, with $g_{n p}=\beta^{2} F^{-1} F^{-T}$ and $g_{n p}^{-1}=\beta^{-2} F^{T} F$. These relationships can be written in terms of the coefficients, i.e. $u_{p s}^{2}=\beta^{-2} u_{t s i}\left(g_{n p}\right)^{i j} u_{t s j}$, $u_{p s}^{2}=\beta^{-2} u_{t s}^{i}\left(g_{n p}^{-1}\right)_{i j} u_{t s}^{j}$ and $\left(g_{n p}^{-1}\right)_{i j}=\beta^{-2} F^{k}{ }_{i} F^{k}{ }_{j}$. It is evident from these identities, that to satisfy $u_{p s}^{2}=\beta^{-2} u_{t s}^{i} u_{t s i}$ it is necessary and sufficient that $u_{t s}^{i}=\left(g_{n p}\right)^{i j} u_{t s j}$ (or $\left.u_{t s i}=\left(g_{n p}^{-1}\right)_{i j} u_{t s}^{j}\right)$. It is clear that the non-physical metric $g_{n p}=\beta^{2} F^{-1} F^{-T}$ has properties identical to a physical metric and under the assumption that $u_{t s}^{3}$ is dominant it follows that

$u_{p s}^{2}=\beta^{-2} u_{t s}^{i}\left(g_{n p}^{-1}\right)_{i j} u_{t s}^{j} \approx \beta^{-2} u_{t s}^{3}\left(g_{n p}^{-1}\right)_{33} u_{t s}^{3} \approx \beta^{-2}\left(g_{n p}^{-1}\right)_{33} u_{t s}^{i} u_{t s}^{i}=\beta^{-2}\left(g_{n p}^{-1}\right)_{33} u_{t s}^{2}$

where $\left(g_{n p}^{-1}\right)_{33}=\beta^{-2} F_{3}^{k} F_{3}^{k}$.

The crux of the approximation is the relationship $u_{t s i} \approx\left(g_{n p}^{-1}\right)_{33} \delta_{i j} u_{t s}^{j}$, which enables the momentum equation (incorporating $\underline{\underline{\sigma}}_{t s}=\underline{\underline{\underline{C}}}_{\mathrm{ts}}: \underline{\underline{\varepsilon}}_{t s}$ ) to be returned to a physical form on setting $\underline{\underline{\underline{C}}}_{t s} \mapsto\left(g_{n p}^{-1}\right)_{33} \underline{\underline{\underline{C}}}_{t s}$ with the understanding that displacement in the $x^{3}$-direction is dominant.

It is recognised the approximation adopted here is a peculiar form of modelling error [29] (termed here similitude error), and as such cannot be removed by means of mesh refinement and time-step reduction in any analysis. However, for the numerical investigations presented in subsequent sections it is shown that the similitude error can be extremely small for a range of problems.

\section{PRACTICAL PROCEDURE}

Despite the apparent complexity associated with the developed theory, it transpires that its application is relatively straightforward. Under the simplification of diagonal $\underline{\underline{F}}$, anisotropic scaling involves six independent scaling factors, which are $\beta_{1}, \beta_{2}, \beta_{3}, g, \alpha^{\rho}$ and $\alpha^{s}$. These scaling factors can be set so that geometrical requirements and selected material properties 
and boundary and initial conditions of any prototype can be fixed on the intended properties [24-28]. The following steps are applied in the trials below:

Step 1. Specify the geometrical and material properties, boundary and initial conditions of the full-scale model;

Step 2. Specify the material properties of the trial model;

Step 3. Specify the independent scaling factors (i.e. $\beta_{1}, \beta_{2}, \beta_{3}, g, \alpha^{\rho}$ and $\alpha^{s}$ in pure thermal processes and $\beta_{1}, \beta_{2}, \beta_{3}, g$ and $\alpha^{\rho}$ in elastic phenomena);

Step 4. Determine the geometrical properties, boundary and loading conditions of the trial model based on the scaling factors calculated in Step 3;

Step 5. Conduct experimental test on the trial model;

Step 6. Reverse the results obtained in Step 5 (reversed trial results) to see how accurately the trial model can replicate the full-scale model in the physical space.

Note that the theory permits projections of the physical-space physics into the trial space but also trial-space physics into the physical space (i.e. reverse trial results).

\section{NUMERICAL TRIALS}

Trialled in this section in three parts (A, B and C) are three different concepts arising from the theory, which are non-physical finite similitude, physical-finite similitude and equivalent isotropic models for aniso/ortho-tropic models.

The first part (Part A), examines non-physical finite similitude for three different case studies; pure thermal, elastostatic deformation and elastodynamic deformation. Note that only one of these case studies (the last one) is truly non-physical requiring explicit involvement of a metric. A schematic diagram of non-physical anisotropic finite similitude and physical anisotropic finite similitude is presented.

The practicality of the physical finite similitude with anisotropic scaling is investigated (in Part B) numerically for two different case studies. The first case study examines the elastic behaviour of a fully-clamped rectangular plate, which is subjected to a transverse dynamic pressure loading. The second considers anisotropic scaling of a simplified airplane wing subjected to transverse dynamic pressure loading.

In Part C, the application of the similitude theory is applied to form equivalent isotropic representations of aniso/ortho-tropic models. This part contains two different case studies for a thermal process and elastodynamic deformation.

In all the examples considered the scaling parameters are selected to minimise global errors as quantified by areas under curves [25-28] for trial and scaled models. The scaled models are virtual models but by minimisation of global errors they attempt to better represent the real models, which involve practical materials (e.g. Aluminium, Steel) selected from a limited set. Zero errors are only possibly if it transpires that the trial and scaled material models match exactly. An additional source of error arises with the appearance of the metric, which for physical-finite similitude is approximated using the theory outlined in Section 7.

\subsection{Part A: Non-physical Finite Similitude}

This part includes three different case studies which are pure thermal, elasto-static deformation and elastodynamic deformation. In each case study it is shown that the response characteristics of an intended model can be predicted using a trial model made of the same or different material despite the geometry being scaled anisotropically. The anisotropic scaling 
of dynamic processes is shown from the perspective of the non-physical theory. The numerical simulation of temperature distribution, elasto-static/dynamic deformation case studies are performed using an in-house finite element code and verified by comparing against the finite element commercial software Abaqus [30]. Unlike Abaqus the in-house code can incorporate a (non-physical) metric and in this context Abaqus plays the role of a physical experiment providing physically realisable predictions.

\subsubsection{Case Study A.I: Pure Thermal Process}

In the following, the surface transient temperature distribution of full-scale, scaled and trial models are obtained using finite element simulation. The in-house finite element code based algorithm presented in Ref. [31] is employed for determining the transient thermal behaviour of the considered case studies. A mesh of 2400 3-node linear heat transfer triangle elements are used for the considered specimens. Also, fixed time increments of $0.005 \mathrm{~s}$ are used, where time integration is based on the backward Euler technique. The finite element software Abaqus with transient heat transfer solver [30] is employed for verification of the full-scale model results.

It is shown through numerical experimentation how a relatively complex geometry such as an ellipse can be scaled anisotropically to a simpler circular geometry and simultaneously an expensive material such as titanium can be replaced by a cheap material such as aluminium. The full-scale elliptic plate under consideration with principal diameters $a_{p s}$ and $b_{p s}\left(a_{p s}\right.$ and $b_{p s}$ in Fig. 4) equal to $0.100 \mathrm{~m}$ and $0.200 \mathrm{~m}$ is made of titanium with boundary and initial temperatures $700 \mathrm{~K}$ and $300 \mathrm{~K}$. The titanium material properties are presented according to Table 2. Using dimensional scaling factors of $\beta_{1}=4$ and $\beta_{2}=2$, respectively in $X$ and $Y$ directions, the elliptic plate is scaled to a circular plate with radius $0.400 \mathrm{~m}$. The circular plate, made of aluminium with properties listed at Table 2, is considered as the first trial model. The scaling factors and equations applied for their determination are listed in Table 3. Note that $\alpha^{\rho}, \rho, \alpha^{s}, c_{p}, u, \alpha^{e}, g$ and $K$ respectively express the density scaling factor, density, entropy scaling factor, specific heat capacity, specific internal energy, energy scaling factor, time scaling factor and thermal conductivity. Application of the scaling factors in Table 3 provides the scaled material properties. The scaled material properties including density, specific heat capacity and thermal conductivities in $x^{1}$ and $x^{2}$ directions are determined to be $2770 \mathrm{~kg} / \mathrm{m}^{3}, 880 \mathrm{~J} / \mathrm{kg} . \mathrm{K}, 140 \mathrm{~W} / \mathrm{m} . \mathrm{K}$ and $35 \mathrm{~W} / \mathrm{m} . \mathrm{K}$, respectively. Anisotropic scaling of geometry leads to scaling of an isotropic thermal conductivity material to an orthotropic thermal conductivity material in which the thermal conductivity in $x^{1}$ and $x^{2}$ directions are respectively obtained using relations $K_{x^{1}}^{\text {Scaled Mat }}=\left(\beta_{1} /\left(\beta_{2} \alpha^{s} g\right)\right) K_{T i}$ and $K_{x^{2}}^{\text {Scaled Mat }}=\left(\beta_{2} /\left(\beta_{1} \alpha^{s} g\right)\right) K_{T i}$ (see Appendix B.2). Also, a circular plate made of Yellow brass with properties listed at Table 2 is considered as the second trial model. A schematic diagram of full-scale and trial models is presented in Fig. 4.

The temperature distribution of full-scale model and the reversed temperature distribution of scaled model, trial model I and trial model II are compared the $s^{1}$-axis path presented in Fig. 5 and the $s^{2}$-axis path according to Fig. 6, at two different instances in time. It is revealed that the full-scale model temperature distribution is predicted with an acceptable accuracy by both trial models I and II. Also, the trial models I and II, made of respectively aluminium and Yellow brass, predict the full-scale model response with approximately the same error. The deviation between the temperature distribution of full-scale and reversed trial models I and II is a consequence of the thermal conductivity not matching in at least one principal direction. 
The cases considered here are designed to capture the overall response, achieved by minimisation of the global error as determined by the areas under the curves [25-28]. However, it is possible to improve on accuracies if so required by adjusting the scaling parameters and selecting alternative trial materials.

Table 2. The material properties of Titanium [32], Aluminium [33], Yellow Brass [34] and Steel [33]

\begin{tabular}{|c|c|c|c|c|c|c|}
\hline \multirow{2}{*}{ Material } & $\begin{array}{c}\rho \\
\left(\mathrm{kg} / \mathrm{m}^{3}\right)\end{array}$ & $\begin{array}{c}c_{p} \\
(\mathrm{~J} / \mathrm{kg} \cdot \mathrm{K})\end{array}$ & $\begin{array}{c}K_{x^{1}} \\
(W / m \cdot K)\end{array}$ & $\begin{array}{c}K_{x^{2}} \\
(W / m \cdot K)\end{array}$ & $v$ & $\begin{array}{c}E \\
(P a) \\
\times 10^{9}\end{array}$ \\
\hline Titanium & 4430 & 670 & 6.600 & 6.600 & 0.330 & 110 \\
\hline Aluminium & 2770 & 880 & 140 & 140 & 0.330 & 70 \\
\hline Yellow Brass & 8470 & 380 & 116 & 116 & - & - \\
\hline Steel & 7830 & 475 & 44.500 & 44.500 & 0.300 & 200 \\
\hline
\end{tabular}

Table 3. Scaling factors of case study A.I

\begin{tabular}{|c|c|c|c|c|c|c|}
\hline & $\beta_{1}$ & $\beta_{2}$ & $\alpha^{\rho}$ & $\alpha^{s}$ & $g$ & $\alpha^{e}$ \\
\hline $\begin{array}{l}\text { Trial Model I } \\
\text { (Aluminium) }\end{array}$ & 4 & 2 & 0.200 & 0.152 & 0.620 & 0.077 \\
\hline $\begin{array}{c}\text { Trial Model II } \\
\text { (Yellow Brass) }\end{array}$ & 4 & 2 & 0.065 & 0.115 & 0.987 & 0.064 \\
\hline $\begin{array}{l}\text { Equation/ } \\
\text { Section }\end{array}$ & - & - & $\begin{array}{c}\alpha^{\rho}=\left(\frac{1}{\beta_{1} \beta_{2}}\right)\left(\frac{\rho_{p s}}{\rho_{t s}}\right) \\
\text { from relation } \\
\rho_{p s}=\alpha^{\rho} \rho_{t s} J \\
(\text { see Table } 1)\end{array}$ & $\begin{array}{c}\alpha^{s}=\alpha^{\rho}\left(\frac{c_{p p s}}{c_{p t s}}\right) \\
\text { from relations } \\
\alpha^{\rho} u_{p s}=\alpha^{e} u_{t s} \\
\text { and } \\
\alpha^{s} T_{p s}=\alpha^{e} T_{t s} \\
(\text { see Section 5) }\end{array}$ & $\begin{array}{l}g=\left(\frac{\beta_{1}}{\beta_{2} \alpha^{s}}\right)\left(\frac{K_{p s}}{K_{t s}}\right) \\
\text { (see Appendix B.2) }\end{array}$ & $\begin{array}{c}\alpha^{e}=\left(\frac{g^{2}}{\beta_{1} \beta_{2}}\right)\left(\frac{\rho_{p s}}{\rho_{t s}}\right) \\
(\text { see Section 5) }\end{array}$ \\
\hline
\end{tabular}

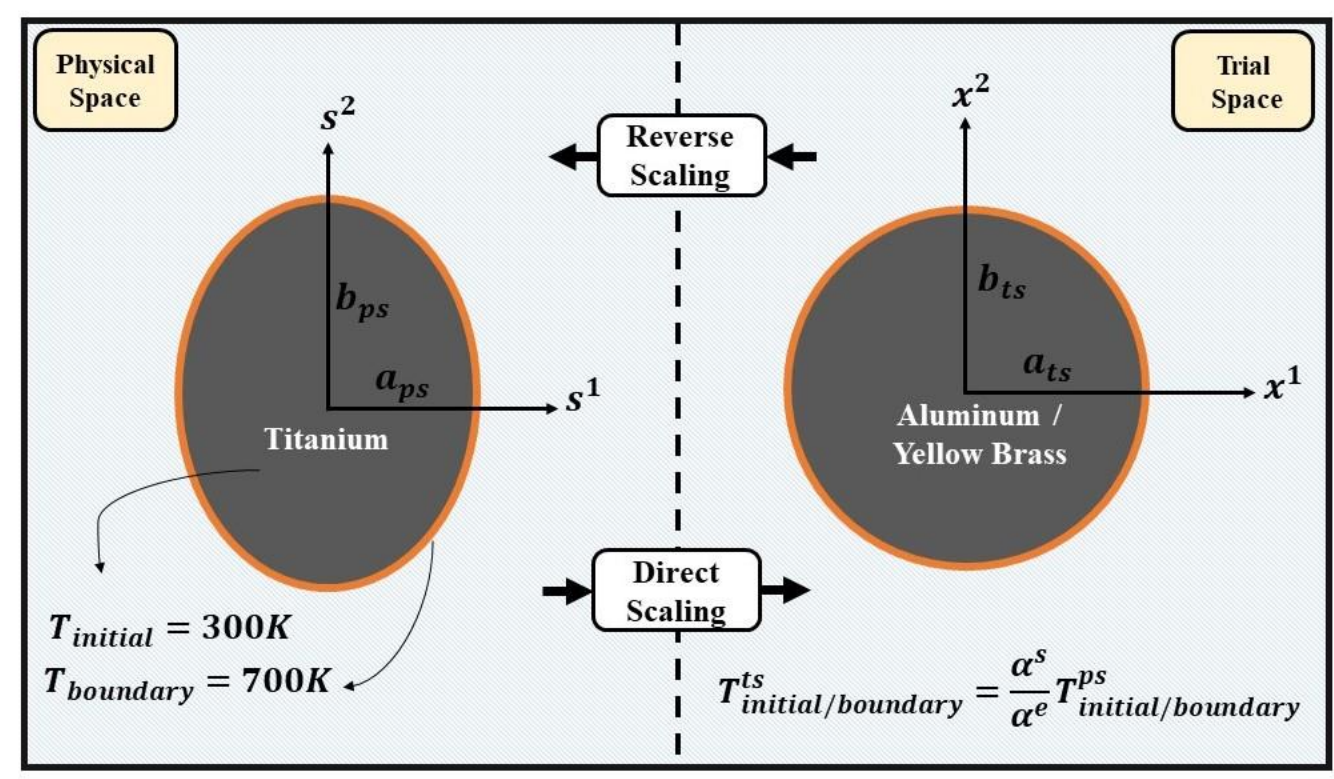

Fig. 4. A schematic diagram representing the scaling of an ellipse to a circle for case study 


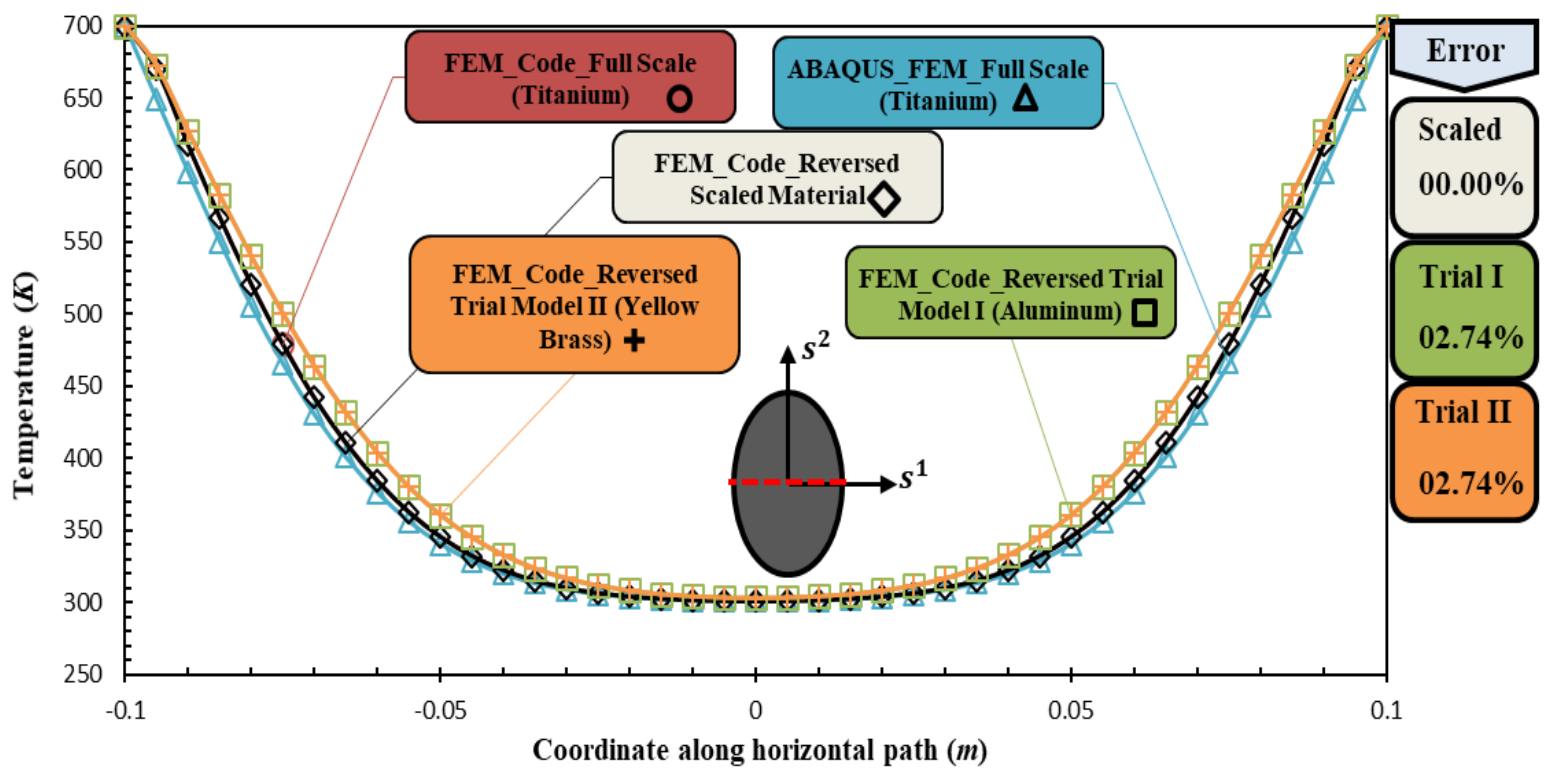

(a) $200 \mathrm{~s}$

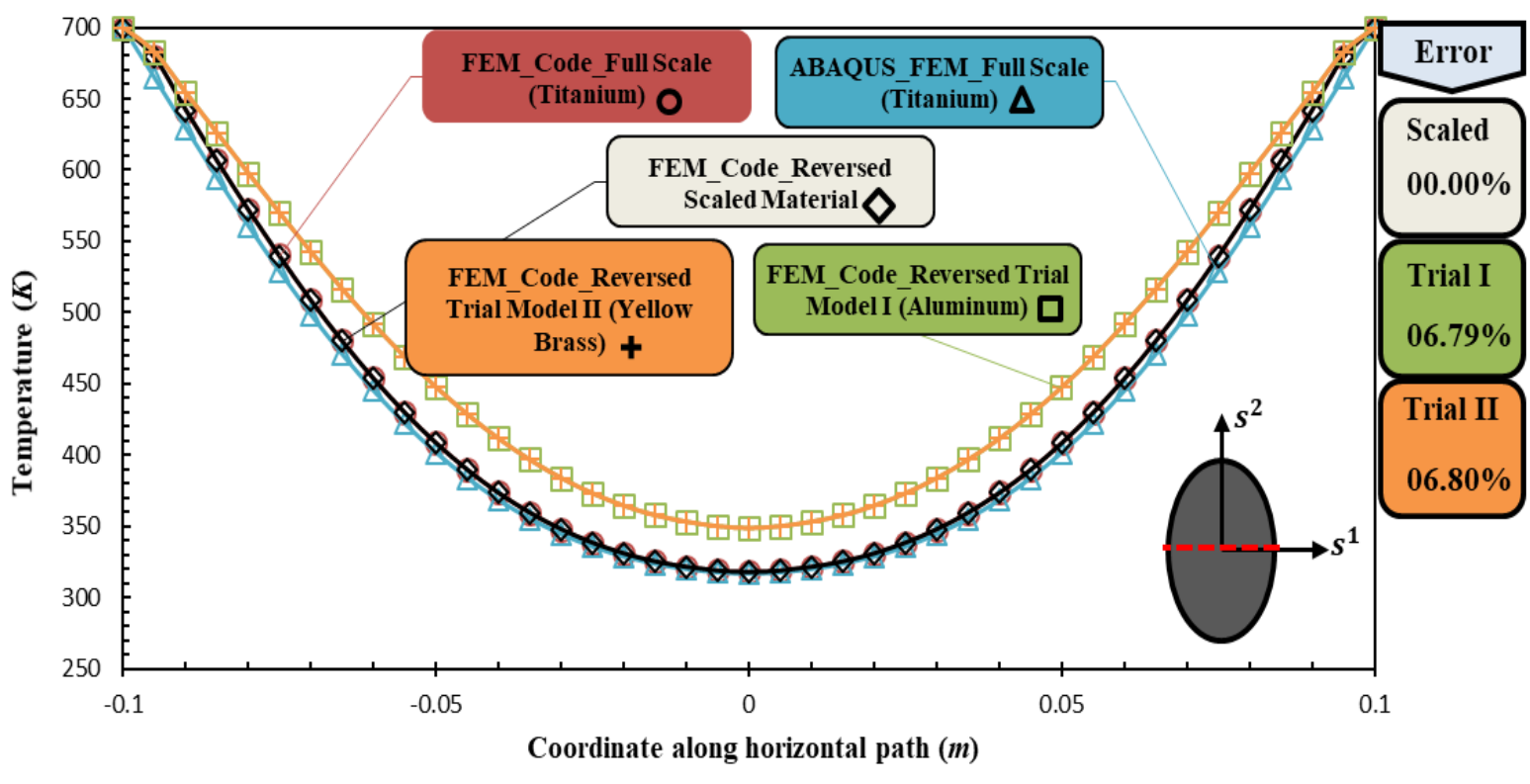

(b) $400 \mathrm{~s}$

Fig. 5. Temperature distribution for an ellipse and a reversed circle along a path labelled by the $s^{1}$-axis at two different times 


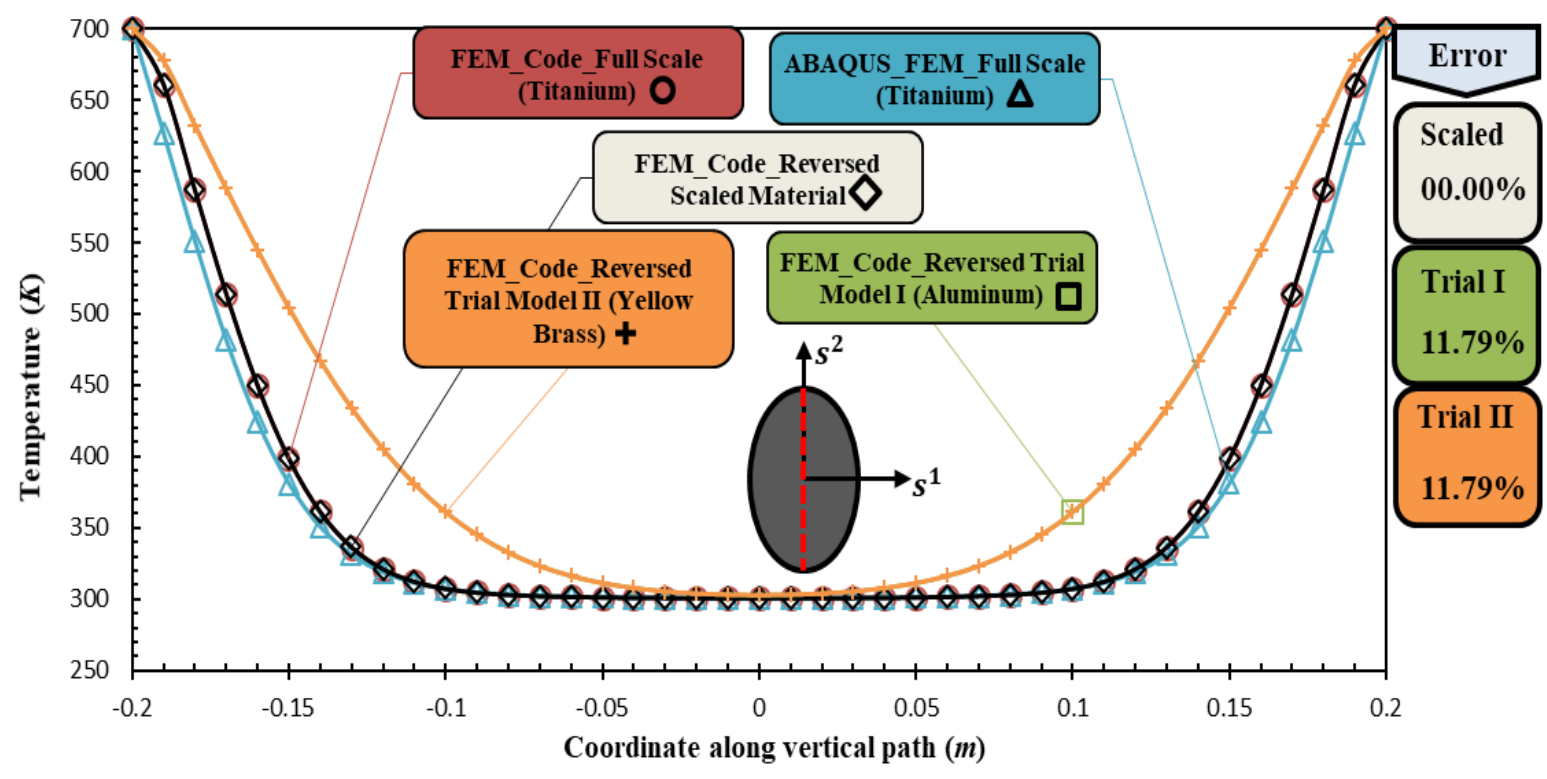

(a) $200 \mathrm{~s}$

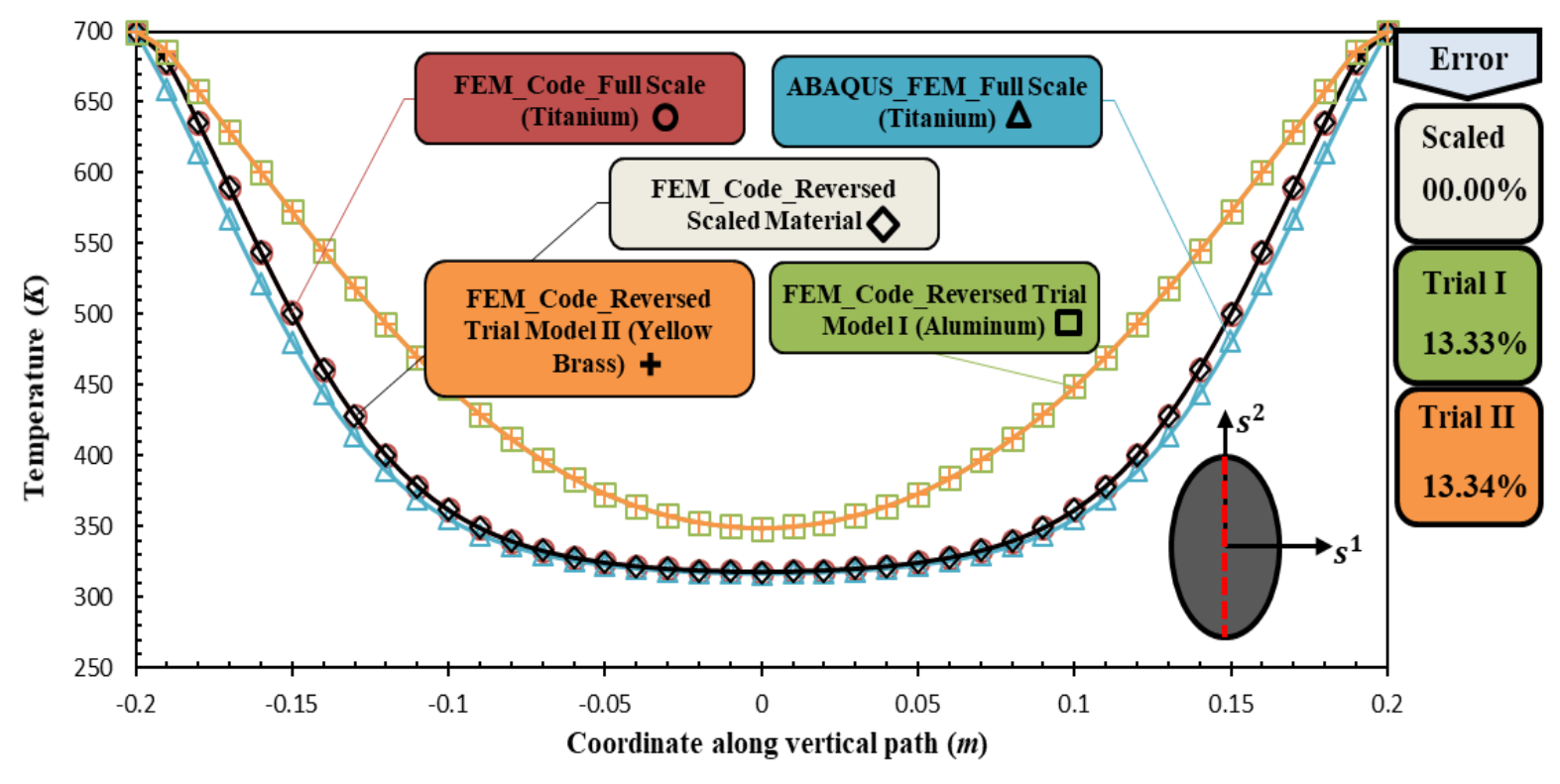

(b) $400 \mathrm{~s}$

Fig. 6. Temperature distribution for an ellipse and a reversed circle along a path labelled by the $s^{2}$-axis at two different times

\subsubsection{Case Study A.II: Elasto-Static Deformation}

Elastostatic deformation of plates is examined with an in-house finite element code based on the algorithm presented in Ref. [35] and is founded on Mindlin plate theory, which accounts for transverse shear deformations. The finite element software Abaqus with static solver [30] is used for verification of the full-scale model. The mesh applied consists of 100 quadrilateral (4-node Q4) and S4R elements for the in-house code and Abaqus software, respectively. It is confirmed numerically that the geometry of a plate structure subjected to static loading can be 
scaled anisotropically with different materials used for the full-scale and trial models. A fully-clamped rectangular plate with $a_{p s}=0.200 \mathrm{~m}, b_{p s}=0.200 \mathrm{~m}$ and $t_{p s}=0.001 \mathrm{~m}\left(a_{p s}, b_{p s}\right.$ and $t_{p s}$ are presented in Fig. 7) is subjected to a transverse static pressure loading of $25000 \mathrm{~Pa}$ and made of an expensive titanium material with properties listed at Table 2. An aluminium rectangular plate with properties listed at Table 2 is considered as the first trial model. In an attempt to predict the full-scale model response with a good accuracy, the stiffness properties $C^{1111}$ and $C^{1212}$ are fixed $\left(C^{1111}\right.$ and $C^{1212}$ dictates the stress in the $X$ direction and in-plane shear stress, respectively). The scaling factors and equations applied to determine them are presented in Table 4. According to presented dimensional scaling factors in Table 4, the dimensions of the rectangular plate is obtained equal to $a_{t s}=0.187 \mathrm{~m}$, $b_{t s}=0.187 \mathrm{~m}$ and $t_{t s}=7.500 \times 10^{-4} \mathrm{~m}$ for the scaled and trial model I. Similarly the transverse static pressure of scaled and trial model $\mathrm{I}$ is obtained to equal $8793 \mathrm{~Pa}$ using the relation $P_{t s}=\left(\beta_{3}^{2} / k\right) P_{p s}$ (Table 1$)$, where $k=\left(g^{2} \rho_{p s}\right) / \rho_{t s}$. The density of scaled material is calculated to be $2770 \mathrm{~kg} / \mathrm{m}^{3}$ and the properties of scaled material are determined using Eq. (B2) in Voigt form as

$$
\left[\begin{array}{cccrl}
78.555 & 25.923 & 0 & 0 & 0 \\
25.923 & 78.555 & 0 & 0 & 0 \\
0 & 0 & 16.869 & 0 & 0 \\
0 & 0 & 0 & 16.869 & 0 \\
0 & 0 & 0 & 0 & 26.316
\end{array}\right] \times 10^{9} \mathrm{~Pa}
$$

Additionally a rectangular plate made of steel with properties listed at Table 2 is considered as the second trial model. A schematic diagram of full-scale and trial models is presented in Fig. 7. The displacement in $s^{3}$-direction, $\sigma^{11}$ and $\sigma^{22}$ of full-scale model and the reversed scaled and trial models are compared on a path parallel to the $s^{1}$-axis according to Fig. 8 and on a path parallel to the $s^{2}$-axis according to Fig. 9 . It is revealed that the response characteristics of full-scale model are predicted with high accuracy by trial model I and with overall errors of less than $7 \%$ by trial model II.

Table 4. Scaling factors of case studies A.II/III

\begin{tabular}{|c|c|c|c|c|}
\hline & $\beta_{1}$ and $\beta_{2}$ & $\beta_{3}$ & $g$ & $\alpha^{\rho}$ \\
\hline $\begin{array}{l}\text { Trial Model I } \\
\text { (Aluminium) }\end{array}$ & 0.937 and 0.937 & 0.750 & 1 & 2.430 \\
\hline $\begin{array}{l}\text { Trial Model II } \\
\text { (Steel) }\end{array}$ & 0.938 and 0.959 & 0.750 & 1 & 0.838 \\
\hline $\begin{array}{l}\text { Equation/ } \\
\text { Section }\end{array}$ & $\begin{array}{c}C_{t s}^{1111}=\left(\frac{|F|^{-2 / D} \beta_{1}^{4}}{k}\right) C_{p s}^{1111} \\
\text { and } \\
C_{t s}^{1212}=\left(\frac{|F|^{-2 / D} \beta_{1}^{2} \beta_{2}^{2}}{k}\right) C_{p s}^{1212} \\
\text { Eq. (B2) }\end{array}$ & - & - & $\begin{array}{c}\alpha^{\rho}=\left(\frac{1}{\beta_{1} \beta_{2} \beta_{3}}\right)\left(\frac{\rho_{p s}}{\rho_{t s}}\right) \\
\text { from relation } \\
\rho_{p s}=\alpha^{\rho} \rho_{t s} J \\
\quad(\text { see Table } 1)\end{array}$ \\
\hline
\end{tabular}




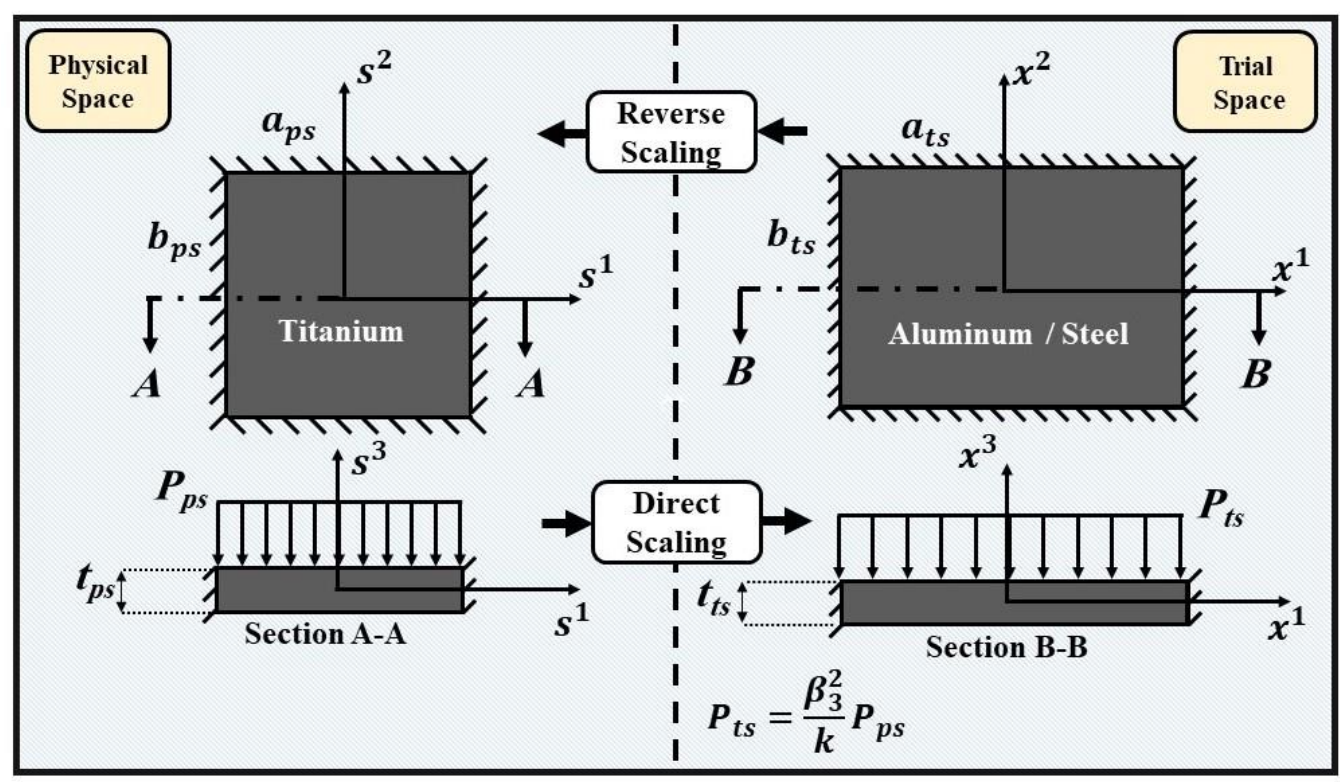

Fig. 7. A schematic diagram representing the scaling of a plate subjected to a transverse pressure for case studies A.II/III and case study B.I

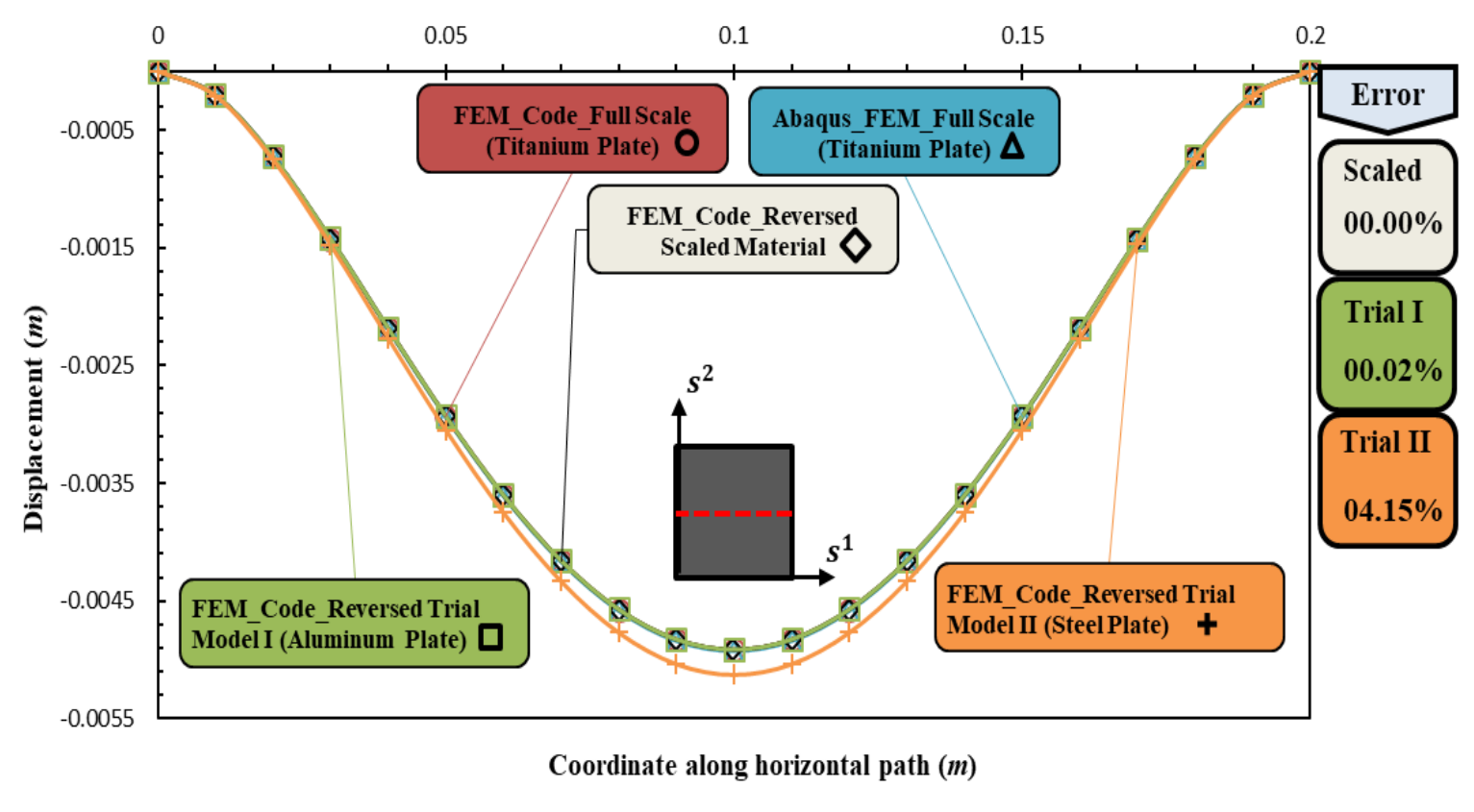

(a) Deflection in $s^{3}$-direction 


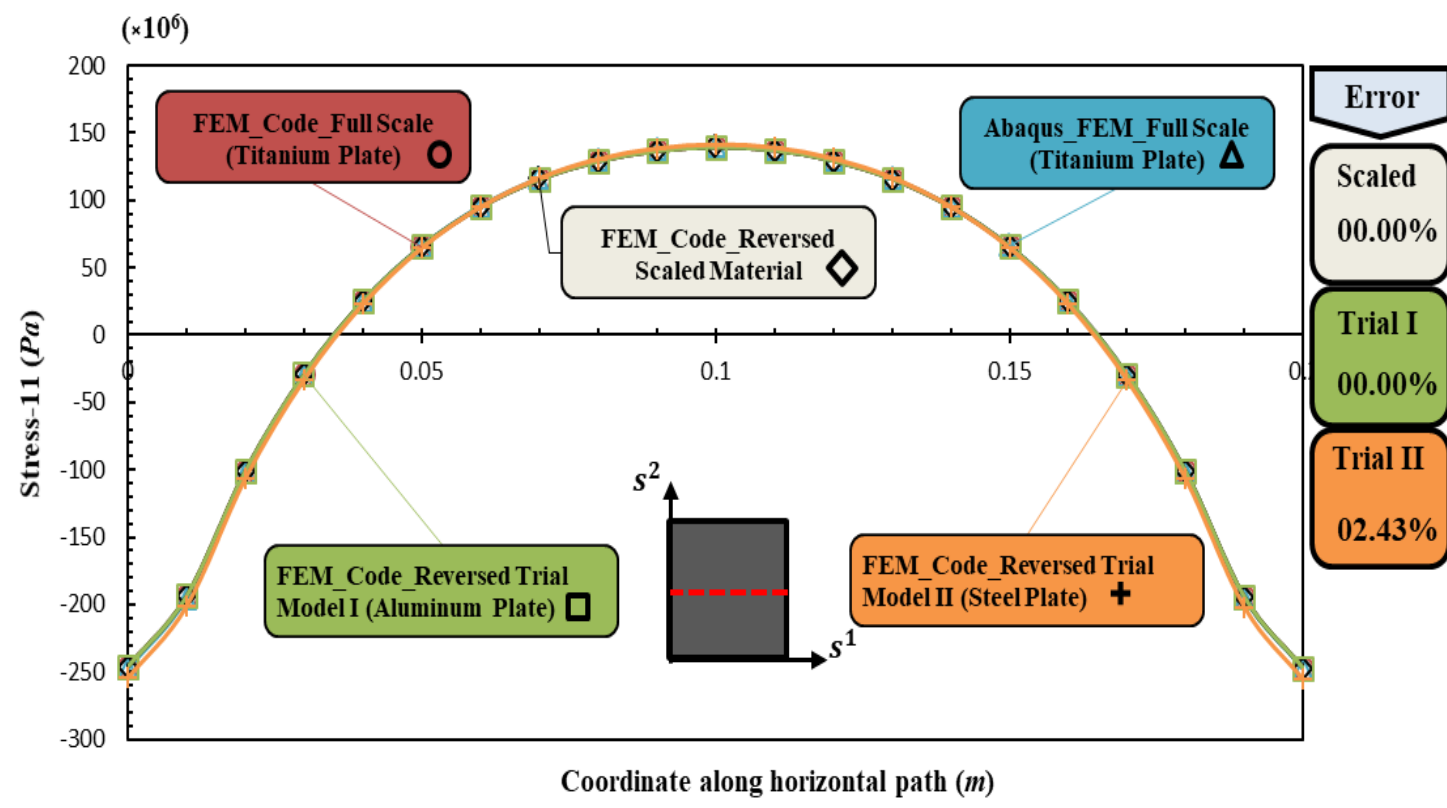

(b) $\sigma^{11}$

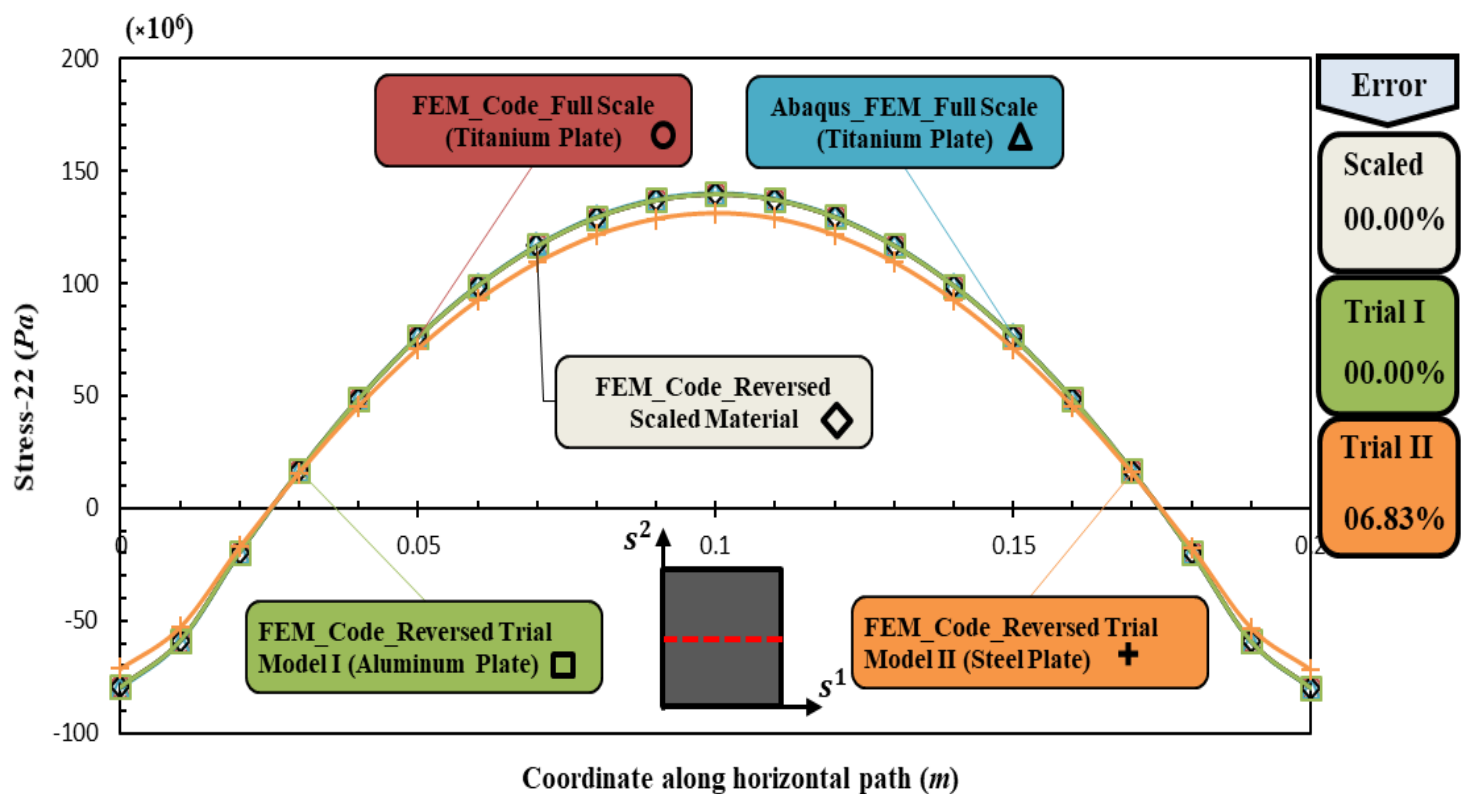

(c) $\sigma^{22}$

Fig. 8. Elastostatic deformation of full-scale and reversed rectangular plates along a path parallel to the $s^{1}$-axis 


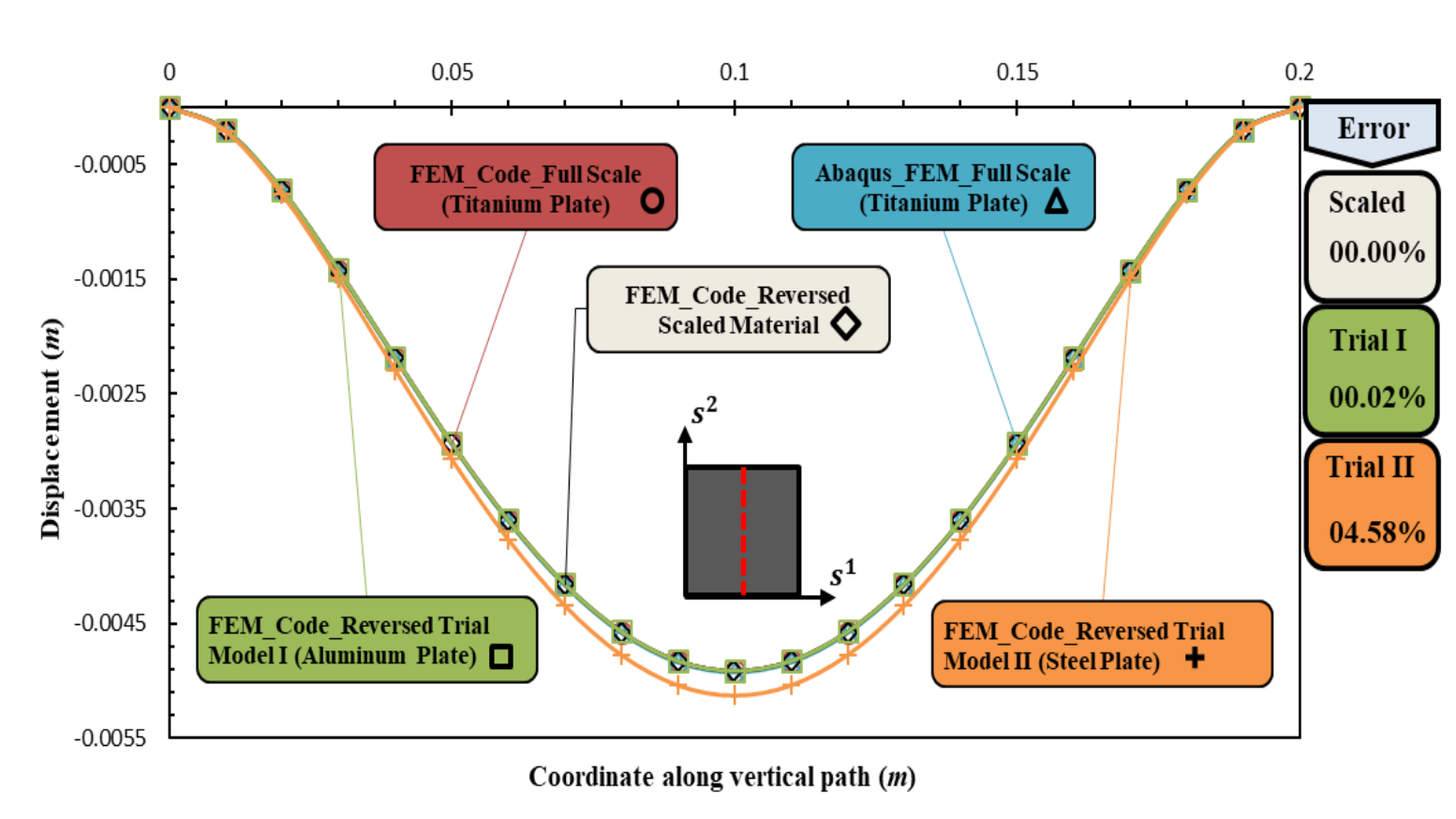

(a) Deflection in $s^{3}$-direction

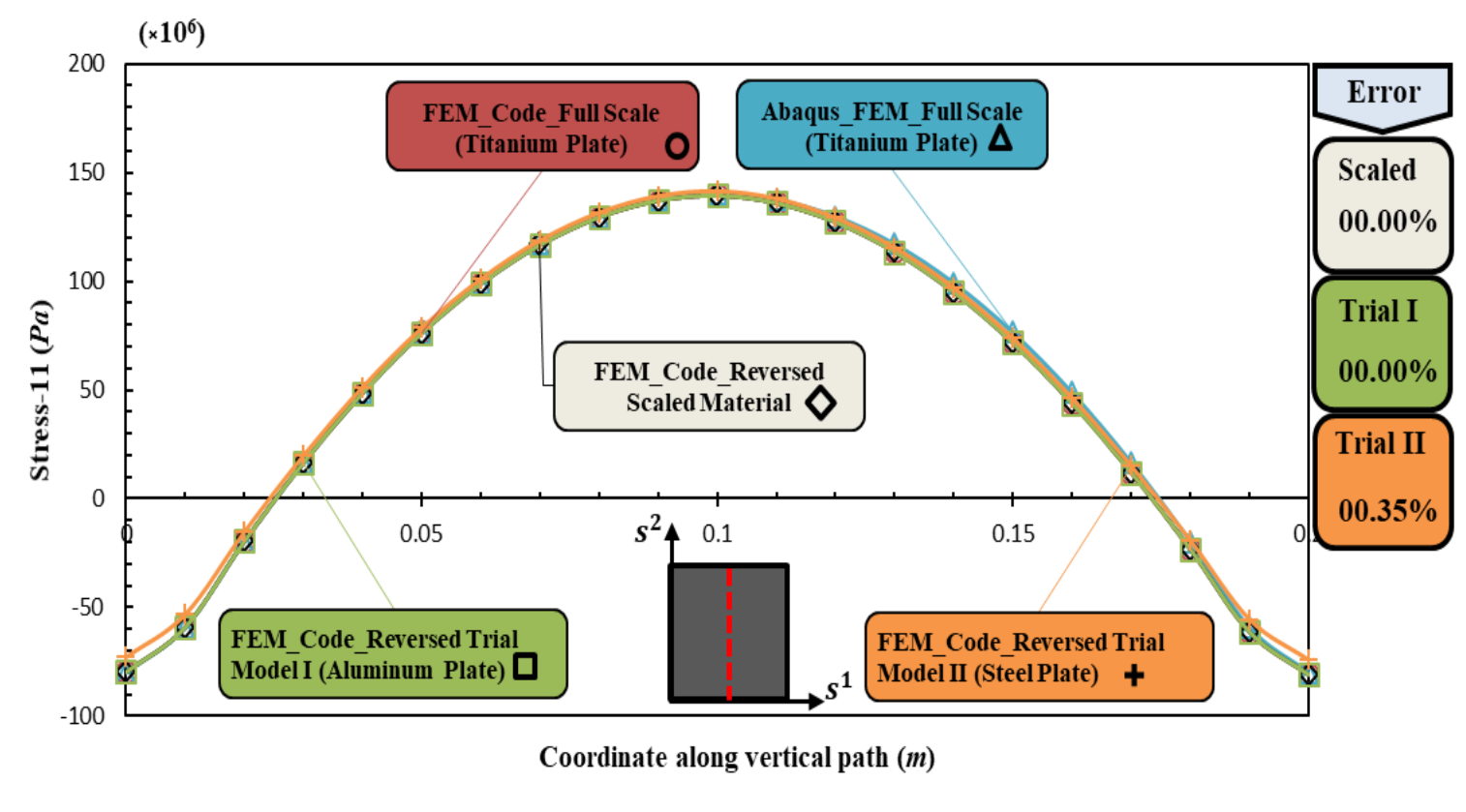

(b) $\sigma^{11}$ 


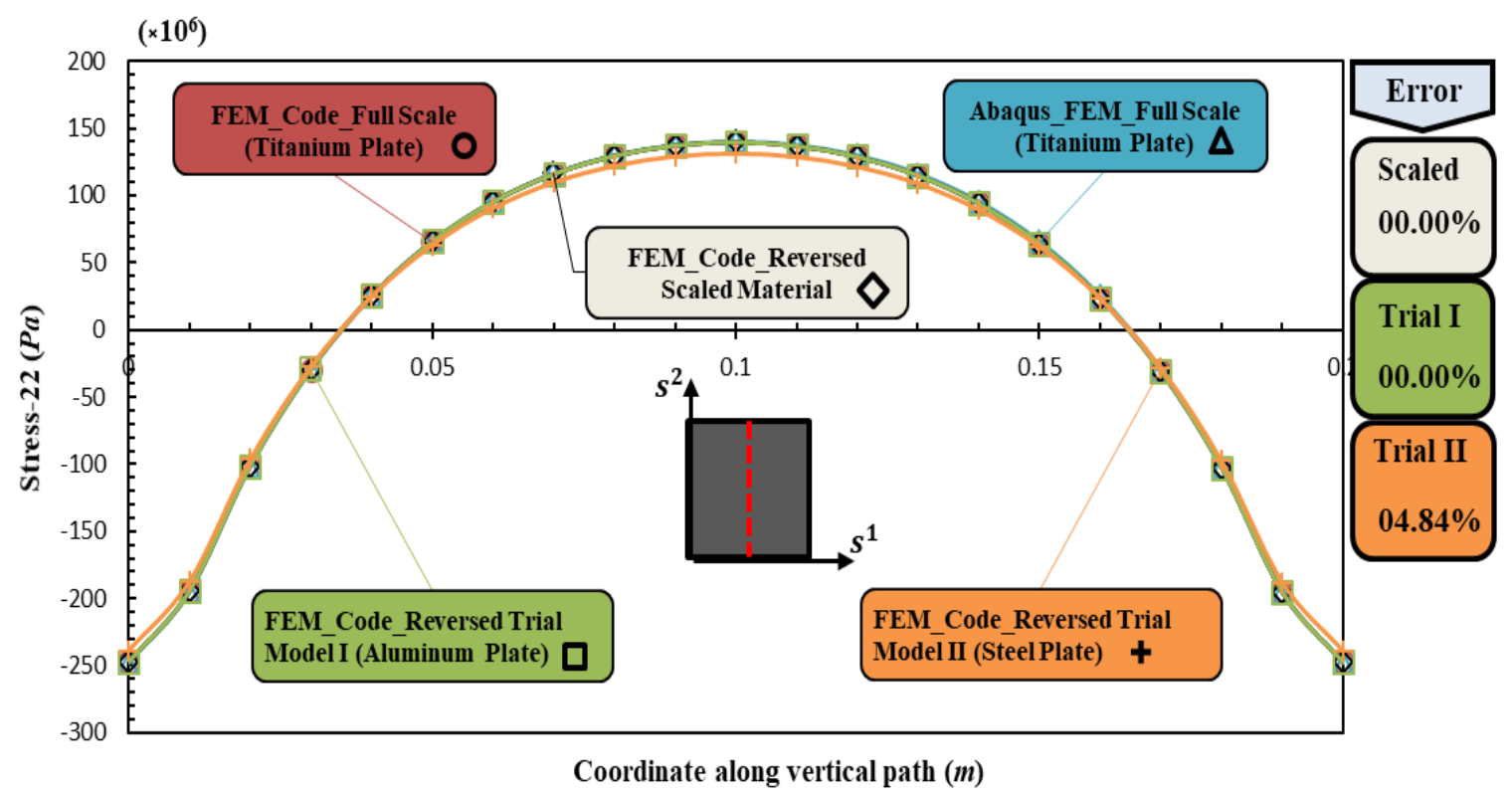

(c) $\sigma^{22}$

Fig. 9. Elastostatic deformation of full-scale and reversed rectangular plates along a path parallel to the $s^{2}$-axis

\subsubsection{Case Study A.III: Elasto-Dynamic Deformation}

Dynamic behaviour of elastic plates are analysed using an in-house finite element code based on Mindlin plate theory with a central difference method for numerical time integration [3536]. A mesh consisting of 400 quadrilateral (4-node Q4) elements are used for spatial discretisation. It should be emphasised that the reversed results of the scaled models involve a non-physical metric. With this proviso in mind, it is shown numerically that the geometry of a structure subjected to dynamic loading can be scaled anisotropically with different materials used for full-scale and trial models. A full-scale, fully-clamped rectangular plate with $a_{p s}=0.200 m, b_{p s}=0.200 m$ and $t_{p s}=0.001 m\left(a_{p s}, b_{p s}\right.$ and $t_{p s}$ are presented in Fig. 7) is subjected to a transverse dynamic pressure loading $25000 \mathrm{~Pa}$ and made a titanium material with properties listed at Table 2. An aluminium rectangular plate with properties tabulated at Table 2 is considered as the first trial model. In an attempt to predict the full-scale model response with a good accuracy, the stiffness properties are fixed. The scaling factors and equations applied for their determination are presented in Table 4. The dimensions of the rectangular plate and the transverse dynamic pressure of scaled and trial model I and the scaled material properties are obtained as in the previous section (Section 9.1.2).

Additionally a rectangular plate made of steel with properties tabulated at Table 2 is considered as the second trial model. A schematic diagram of full-scale and trial models is presented according to Fig. 7. Temporal displacement in the $s^{3}$-direction, $\sigma^{11}$ and $\sigma^{22}$ of full-scale and reversed scaled and trial models are compared at the centre of the plate according to Fig. 10. It is revealed that the response characteristics of full-scale model are predicted with high accuracy by trial model I and with overall errors of less than $6 \%$ by trial model II. 


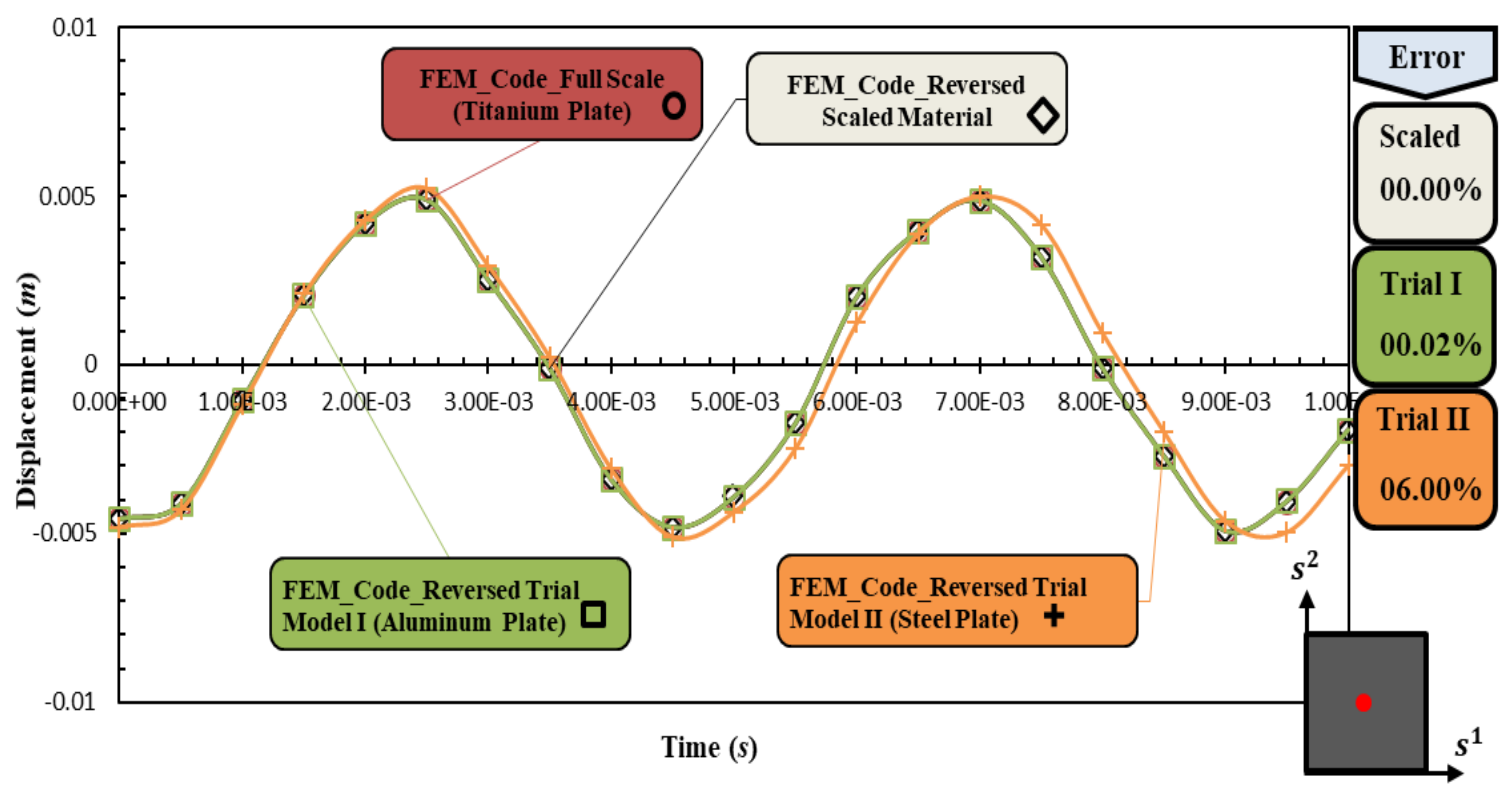

(a) Deflection in $s^{3}$-direction

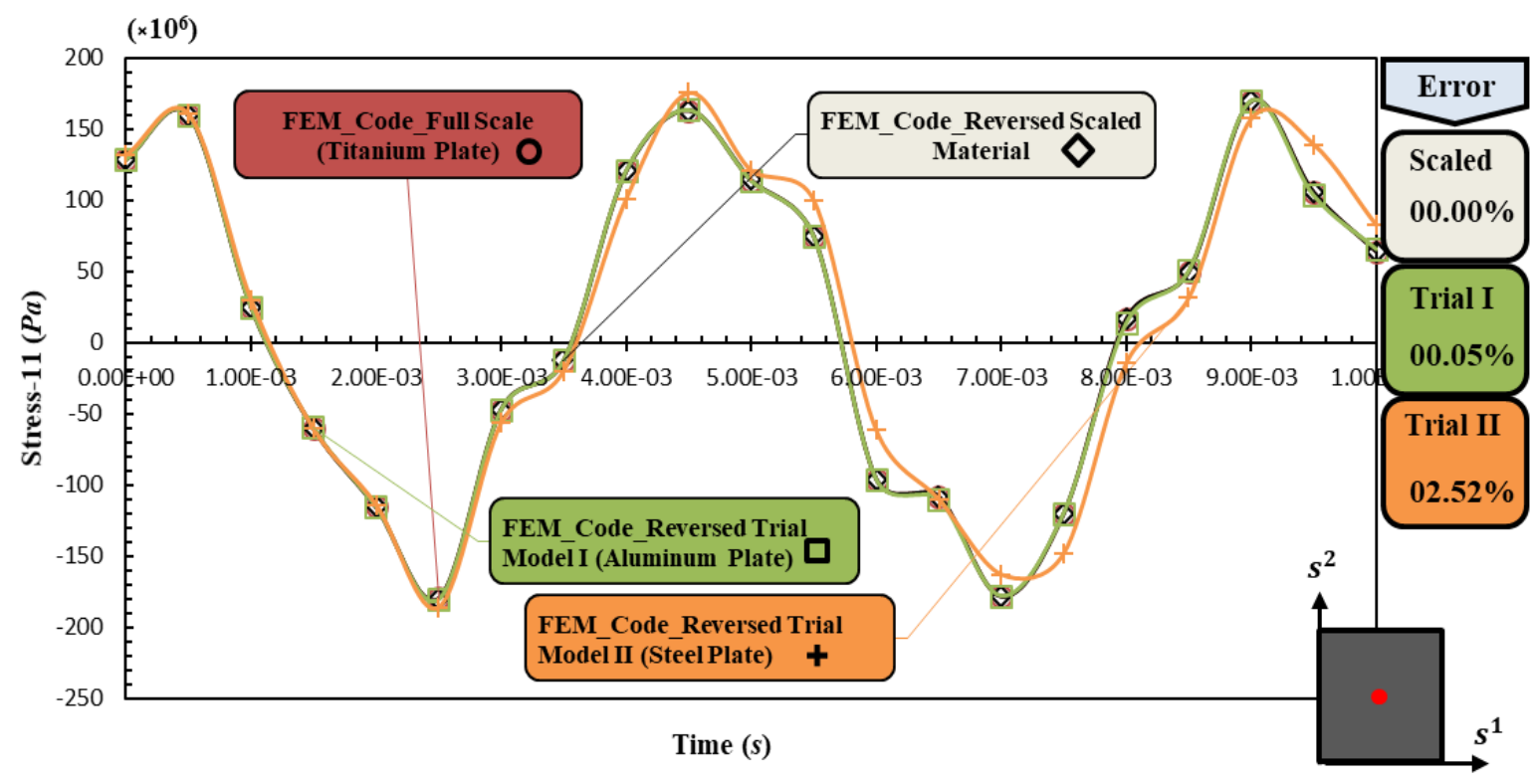

(b) $\sigma^{11}$ 


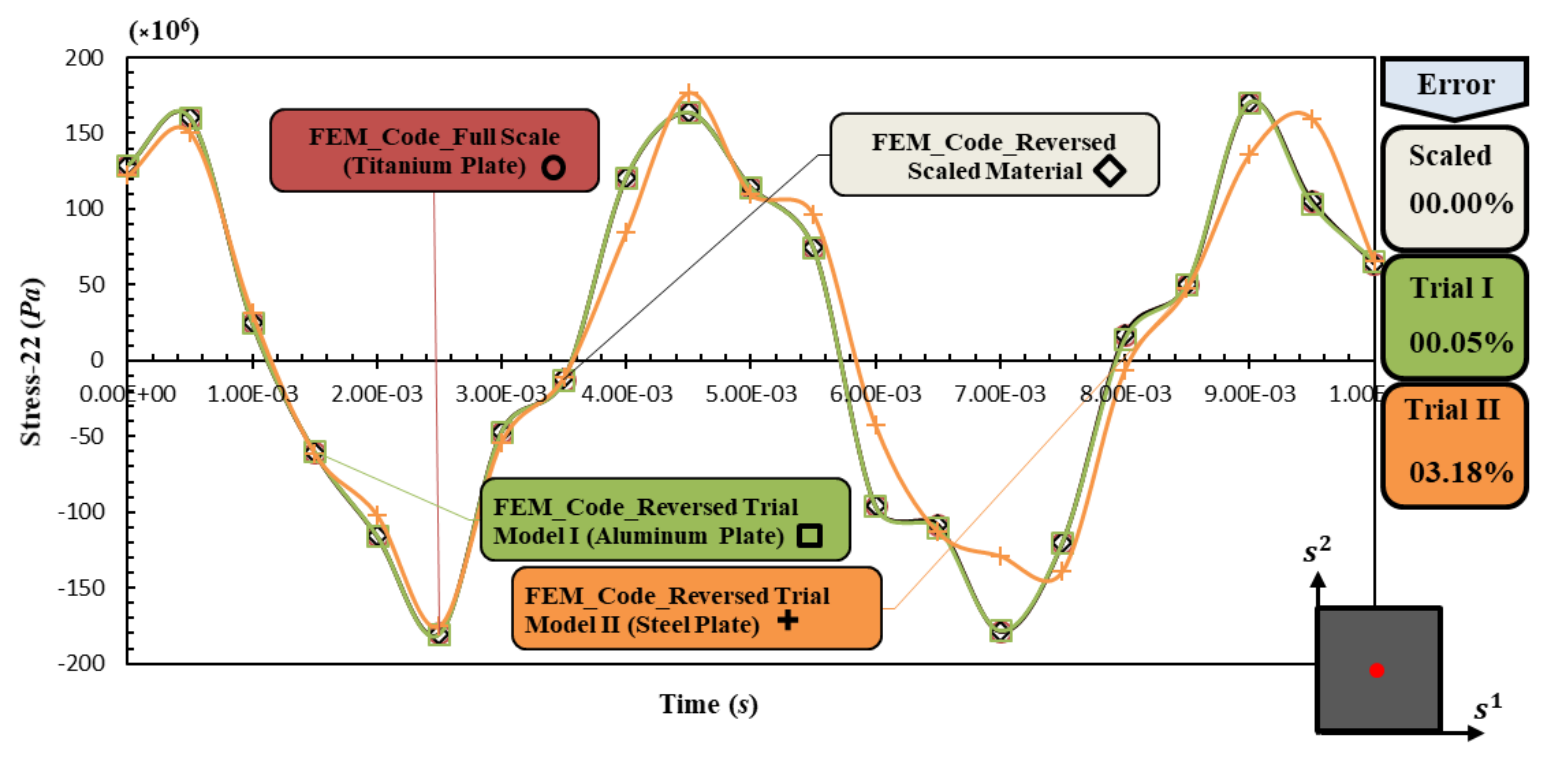

(c) $\sigma^{22}$

Fig. 10. Elastodynamic deformation of full-scale and reversed rectangular plates at a central point and involving the metric using an in-house code

\subsection{Part B: Physical Anisotropic Finite Similitude}

Anisotropic space scaling of dynamic processes can lead to non-physical finite similitude involving a non-physical metric and consequently to a degree of impracticality. Under certain conditions however problems can be made physically realisable despite the involvement of the metric. This is termed physical-finite similitude, which is tested here on a plate and a simplified airplane wing subjected to the dynamic transverse pressure loading.

\subsubsection{Case Study B.I: Elasto-Dynamic Deformation of Plate}

It is shown here that the geometry and material of a structure subjected to dynamic loading can be scaled simultaneously using the introduced physical-finite similitude. The geometry can be scaled with different ratios in different directions and different materials can be used for full-scale and trial models. The physical-finite similitude theory in dynamic deformation is applicable (see Section 9.1), where a dominant direction exists. The efficacy and application of the proposed method is investigated here on a fully-clamped rectangular plate using the commercial finite element software Abaqus [30]. The dynamic explicit solver [30] is employed for simulation of plates in which a mesh of $400 \mathrm{~S} 4 \mathrm{R}$ elements is used. The fullyclamped rectangular plate with properties listed at Sections 9.1.2 and 9.1.3 is considered here as the full-scale model. An aluminium rectangular plate with properties listed at Table 2 is considered as the first trial model. Stiffness properties are fixed in a first attempt at predicting the full-scale model response to good accuracy. The scaling factors and equations applied for their determination are presented in Table 5. The dimensions of the scaled rectangular plate is obtained and are $a_{t s}=0.174 \mathrm{~m}, b_{t s}=0.174 \mathrm{~m}$ and $t_{t s}=7.500 \times 10^{-4} \mathrm{~m}$, and the transverse dynamic pressure is $10715 \mathrm{~Pa}$. The density of scaled material is obtained equal to $2770 \mathrm{~kg} / \mathrm{m}^{3}$ and the properties of scaled material are determined using Eq. (B2) in Voigt form as 


$$
\left[\begin{array}{cccrc}
78.555 & 25.923 & 0 & 0 & 0 \\
25.923 & 78.555 & 0 & 0 & 0 \\
0 & 0 & 19.564 & 0 & 0 \\
0 & 0 & 0 & 19.564 & 0 \\
0 & 0 & 0 & 0 & 26.316
\end{array}\right] \times 10^{9} \mathrm{~Pa}
$$

Additionally a rectangular plate made of steel with properties tabulated at Table 2 is considered as the second trial model. A schematic diagram of full-scale and trial models is presented according to Fig. 7. Temporal displacement in the $s^{3}$-direction, $\sigma^{11}$ and $\sigma^{22}$ of the full-scale and the reversed scaled and trial models are compared at the centre of the plates according to Fig. 11. It is revealed that the response characteristics of full-scale model are predicted with high accuracy by trial model I and with overall errors of less than $5.60 \%$ by trial model II. The reverse of the scaled model might be expected to provide no error as is the case for the tests above but a very small modelling error (similitude error) is present in this case as revealed by the results in Fig. 11.

Table 5. Scaling factors of case study B.I

\begin{tabular}{|c|c|c|c|c|}
\hline & $\beta_{1}$ and $\beta_{2}$ & $\beta_{3}$ & $g$ & $\alpha^{\rho}$ \\
\hline $\begin{array}{l}\text { Trial Model I } \\
\text { (Aluminum) }\end{array}$ & 0.870 and 0.870 & 0.750 & 1 & 2.818 \\
\hline $\begin{array}{l}\text { Trial Model II } \\
\text { (Steel) }\end{array}$ & 0.868 and 0.887 & 0.750 & 1 & 0.981 \\
\hline $\begin{array}{l}\text { Equation } \\
\text { /Section }\end{array}$ & $\begin{array}{c}C_{t s}^{1111}=\left(\frac{|F|^{-2 / D} \beta_{1}^{4}}{k}\right) C_{p s}^{1111} \\
\text { and } \\
C_{t s}^{1212}=\left(\frac{|F|^{-2 / D} \beta_{1}^{2} \beta_{2}^{2}}{k}\right) C_{p s}^{1212} \\
\text { Eq. (B2) }\end{array}$ & - & - & $\begin{array}{c}\alpha^{\rho}=\left(\frac{1}{\beta_{1} \beta_{2} \beta_{3}}\right)\left(\frac{\rho_{p s}}{\rho_{t s}}\right) \\
\text { from relation } \\
\rho_{p s}=\alpha^{\rho} \rho_{t s} J \\
\quad(\text { see Table 5) }\end{array}$ \\
\hline
\end{tabular}




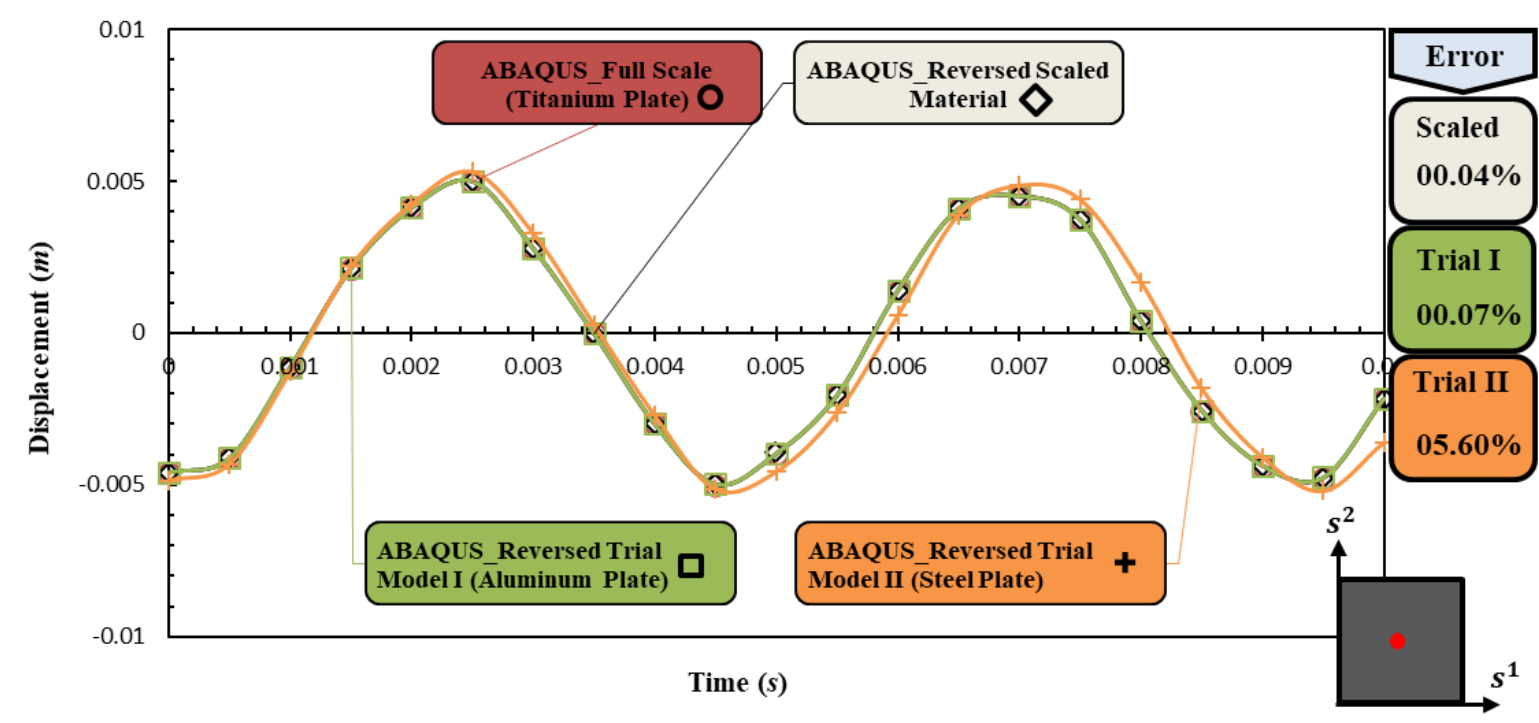

(a) Deflection in $s^{3}$-direction

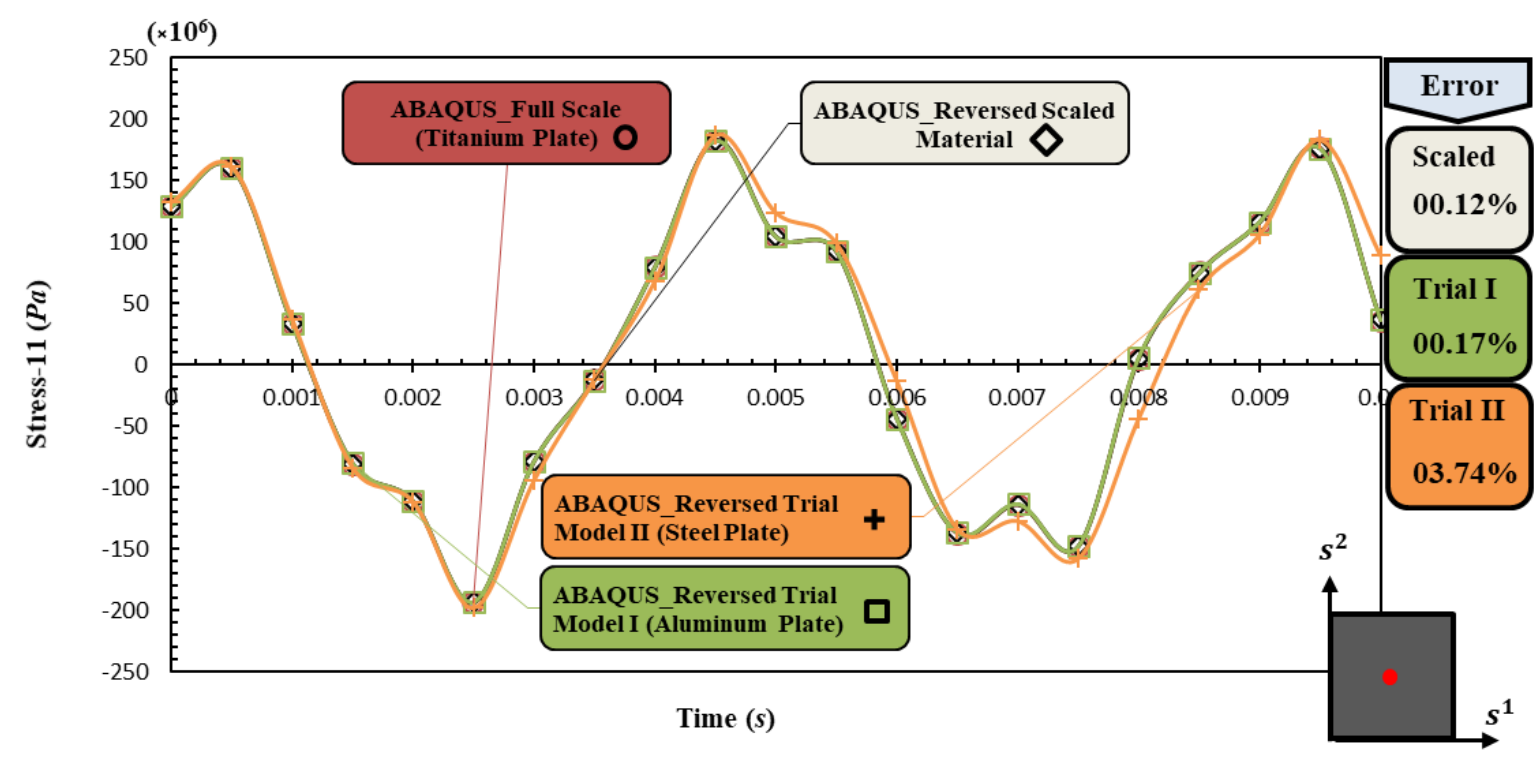

(b) $\sigma^{11}$ 


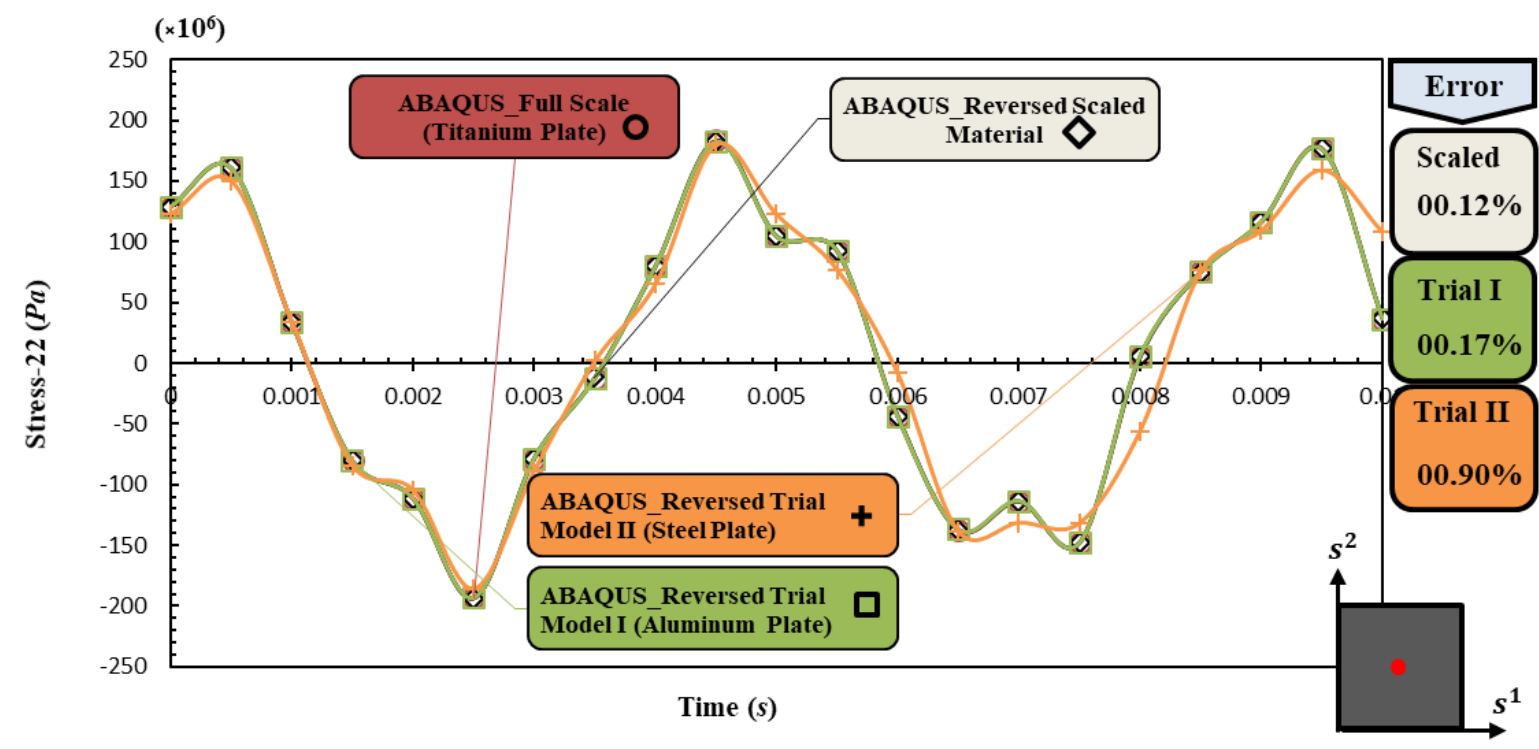

(c) $\sigma^{22}$

Fig. 11. Elastodynamic deformation of full-scale and reversed rectangular plates at a central point and involving the metric in a dominant direction in Abaqus

\subsubsection{Case Study B.II: Elasto-Dynamic Deformation of an Airplane Wing}

Demonstrated here is the practicality of the introduced physical finite similitude on a huge structure such as an airplane wing. It is showed numerically that the wing of an airplane can be scaled with different geometrical ratios in different directions and simultaneously different materials can be used for full-scale and trial models. The numerical simulation is performed using the commercial finite element software Abaqus [30] by employing the explicit solver [30] in which 400 S4R elements are used for discretization of the wing.

A simplified airplane wing made of aluminium with material properties tabulated in Table 2 and with dimensions depicted at Fig. 12 [37-39] is considered as the full-scale model. The thickness of airplane wing is considered here to be a constant value for simplicity. Also a simplified airplane wing made of titanium with listed properties at Table 2 and with dimensions according to Fig. 12 is considered as the first trial model. The dimensions of the wing as a trial model are based on the depicted dimensional scaling factors according to Fig. 12 and Table 6. The scaling factors applied along with the equations used for their determination are tabulated in Table 6. Also, the applied transverse pressure on the upper and lower surfaces of the full-scale airplane wing is depicted in Fig. 13 [37-39], where the transverse dynamic pressure of scaled and trial models is obtained using $P_{t s}=\left(\beta_{3}^{2} / k\right) P_{p s}$, where $k=\left(g^{2} \rho_{p s}\right) / \rho_{t s}$. The density of scaled material is obtained equal to $4430 \mathrm{~kg} / \mathrm{m}^{3}$ and the properties of scaled material are determined using Eq. (B2) in Voigt form as

$\left[\begin{array}{cccrl}123.443 & 40.736 & 0 & 0 & 0 \\ 40.736 & 123.443 & 0 & 0 & 0 \\ 0 & 0 & 165.414 & 0 & 0 \\ 0 & 0 & 0 & 165.414 & 0 \\ 0 & 0 & 0 & 0 & 41.353\end{array}\right] \times 10^{9} \mathrm{~Pa}$


Additionally a fully-clamped simplified airplane wing made of steel with tabulated properties in Table 2 is considered as the second trial model. A schematic diagram of full-scale and trial models is presented in Fig. 12. Temporal displacement in the $s^{3}$-direction at a specified point, $\sigma^{11}$ and $\sigma^{22}$ on a specified path, along with $\varepsilon^{22}$ (strain in the $s^{2}$ direction) of full-scale model and reversed scaled and trial models are compared in Fig. 14. It is revealed that in all cases, the full-scale response characteristics are predicted with good accuracy (less than 3.2\% for scaled and trial model I and less than $10 \%$ for trial model II). Also, the von Mises stress distribution of full-scale and first trial airplane wings are compared at two different instances in time according to Fig. 15, which shows a close agreement between them.

Observe that the airplane wings with properties listed at Table 6, involve a time scaling factor (i.e. $g$ ) is not equal to 1 , which indicates that the full-scale and trial models can be run at different rates. Physical finite similitude can be used for case studies which have a dominant direction but equally good approximations can be obtained involving more complex behaviour such as a combination of displacement and rotation as seen in an airplane wing.

Table 6. Scaling factors of case study B.II

\begin{tabular}{|c|c|c|c|c|c|}
\hline & $\beta_{1}$ & $\beta_{2}$ & $\beta_{3}$ & $g$ & $\alpha^{\rho}$ \\
\hline $\begin{array}{c}\text { Trial Model I } \\
\text { (Titanium) }\end{array}$ & 0.250 & 0.250 & 0.500 & 0.126 & 20.009 \\
\hline $\begin{array}{c}\text { Trial Model II } \\
\text { (Steel) }\end{array}$ & 0.250 & 0.250 & 0.500 & 0.126 & 11.321 \\
\hline $\begin{array}{c}\text { Equation/ } \\
\text { Section }\end{array}$ & - & - & - & $C_{t s}^{1111}=\left(\frac{|F|^{-2 / D} \beta_{1}^{4}}{k}\right) C_{p s}^{1111}$ & $\alpha^{\rho}=\left(\frac{1}{\beta_{1} \beta_{2} \beta_{3}}\right)\left(\frac{\rho_{p s}}{\rho_{t s}}\right)$ \\
or \\
from relation \\
$\rho_{p s}=\alpha^{\rho} \rho_{t s} J$ \\
(see Table 5)
\end{tabular}

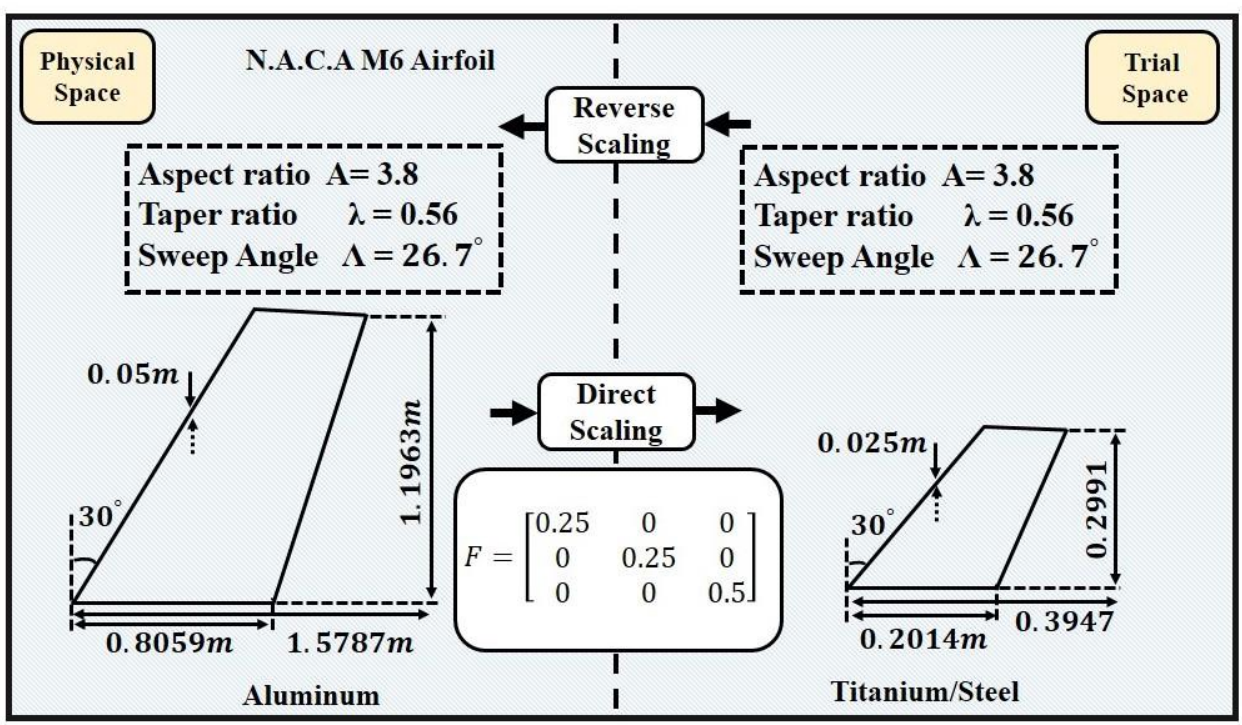

Fig. 12. A schematic diagram representing the scaling of an airplane wing for case study B.II 


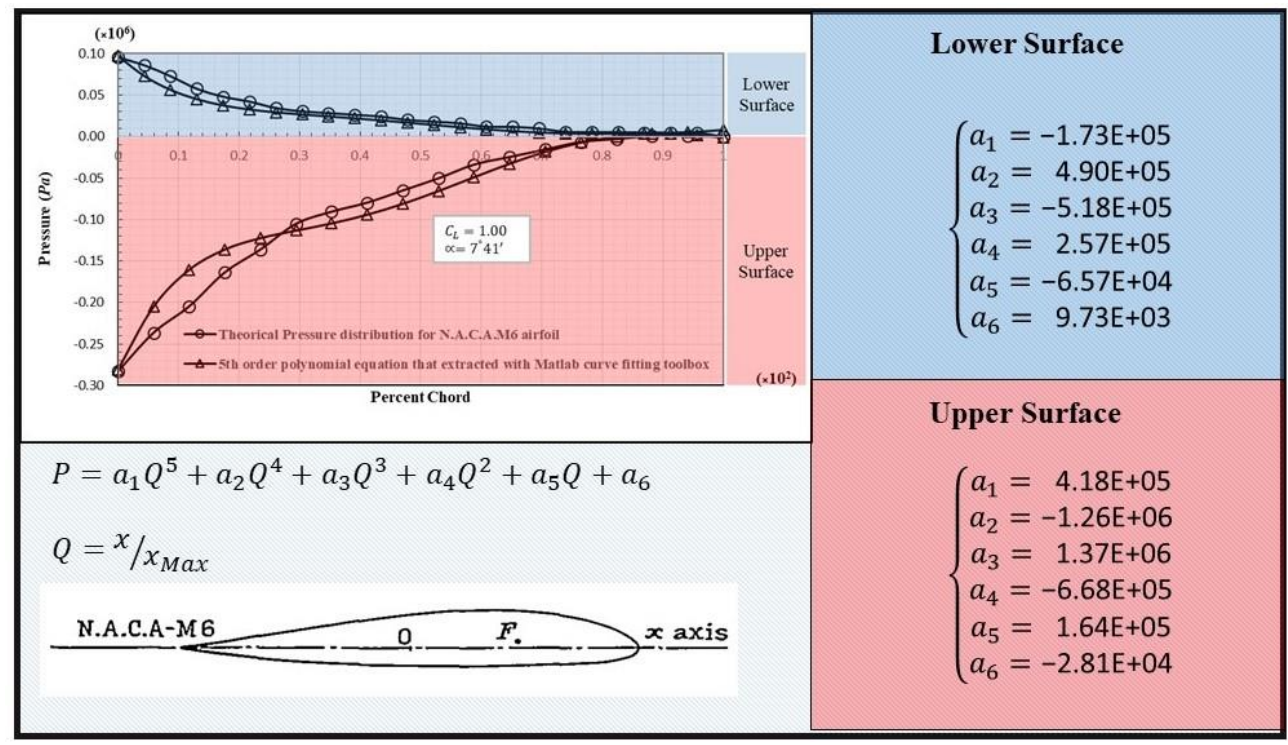

Fig. 13. The applied transverse pressure on the upper and lower surfaces of the full-scale airplane wing [37-39]

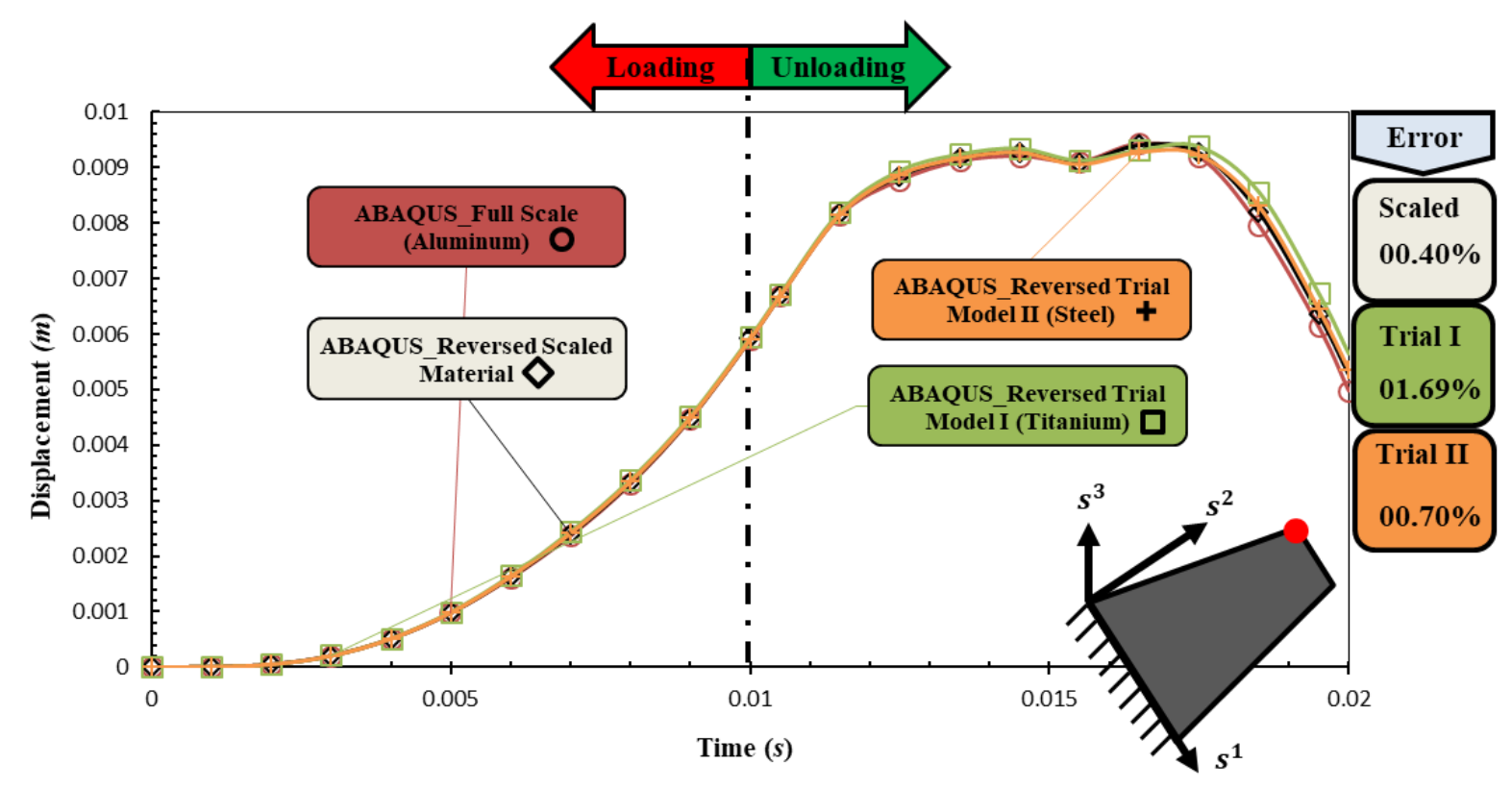

(a) Deflection in $s^{3}$-direction 


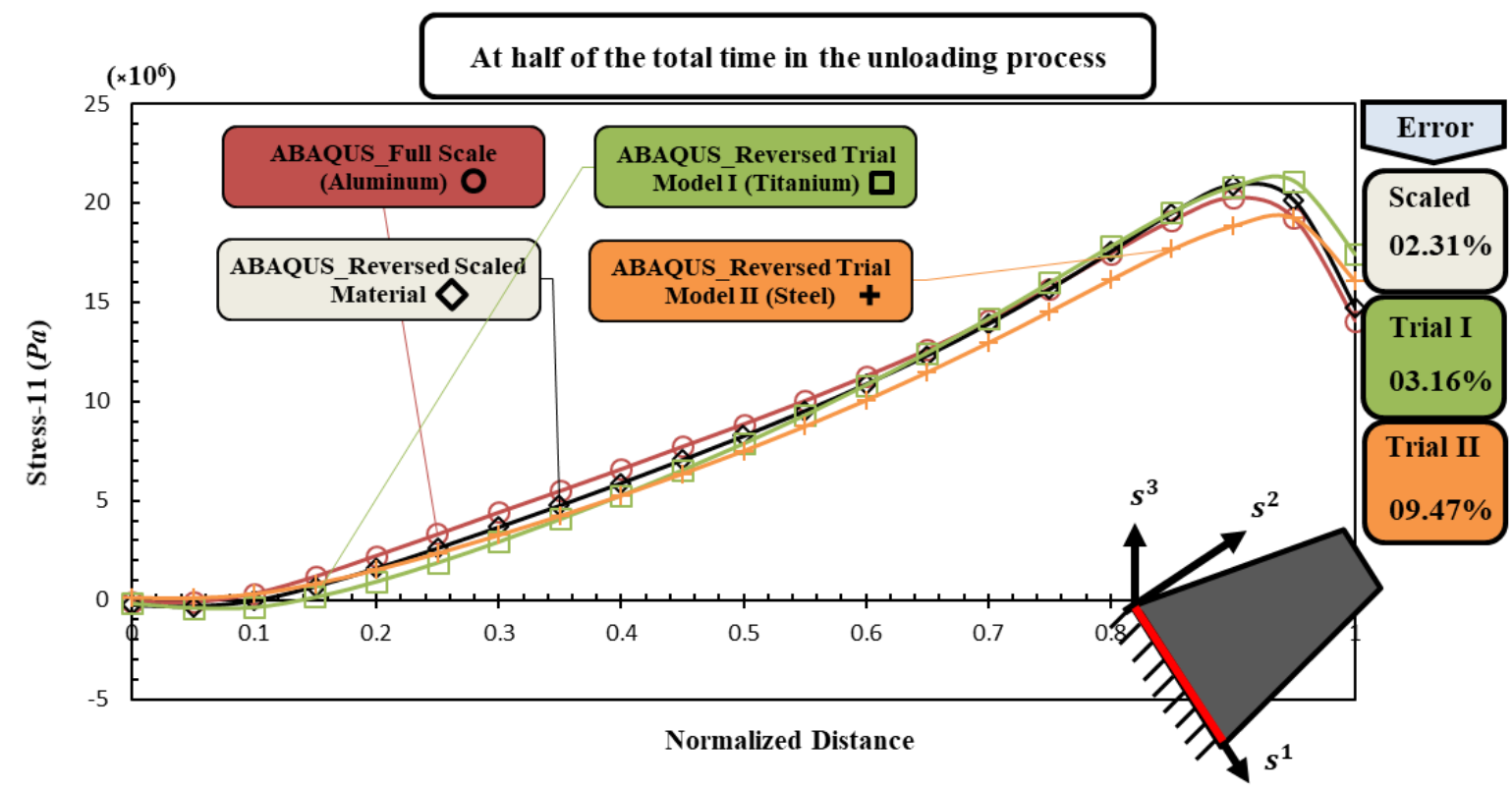

(b) $\sigma^{11}$

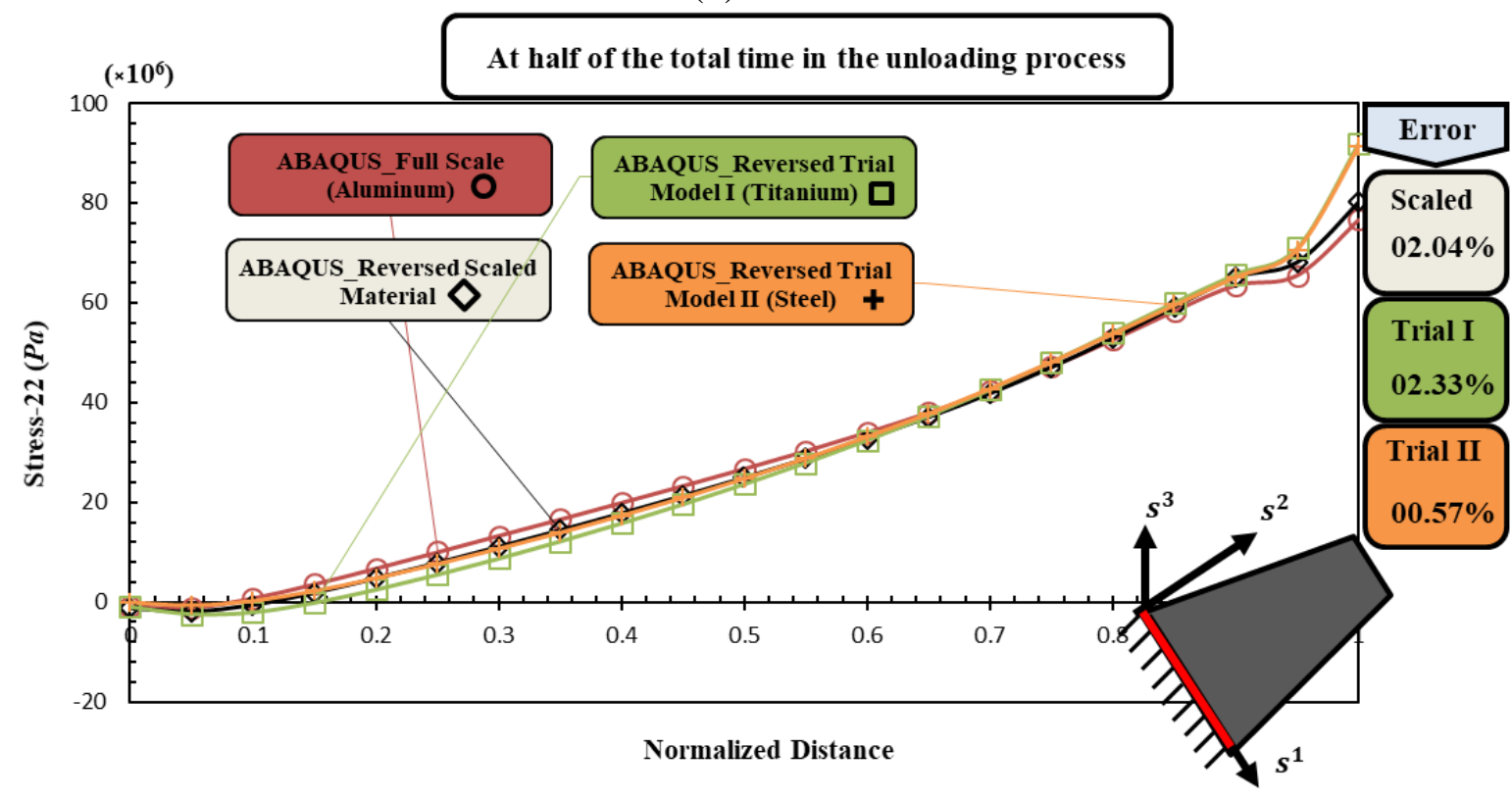

(c) $\sigma^{22}$ 


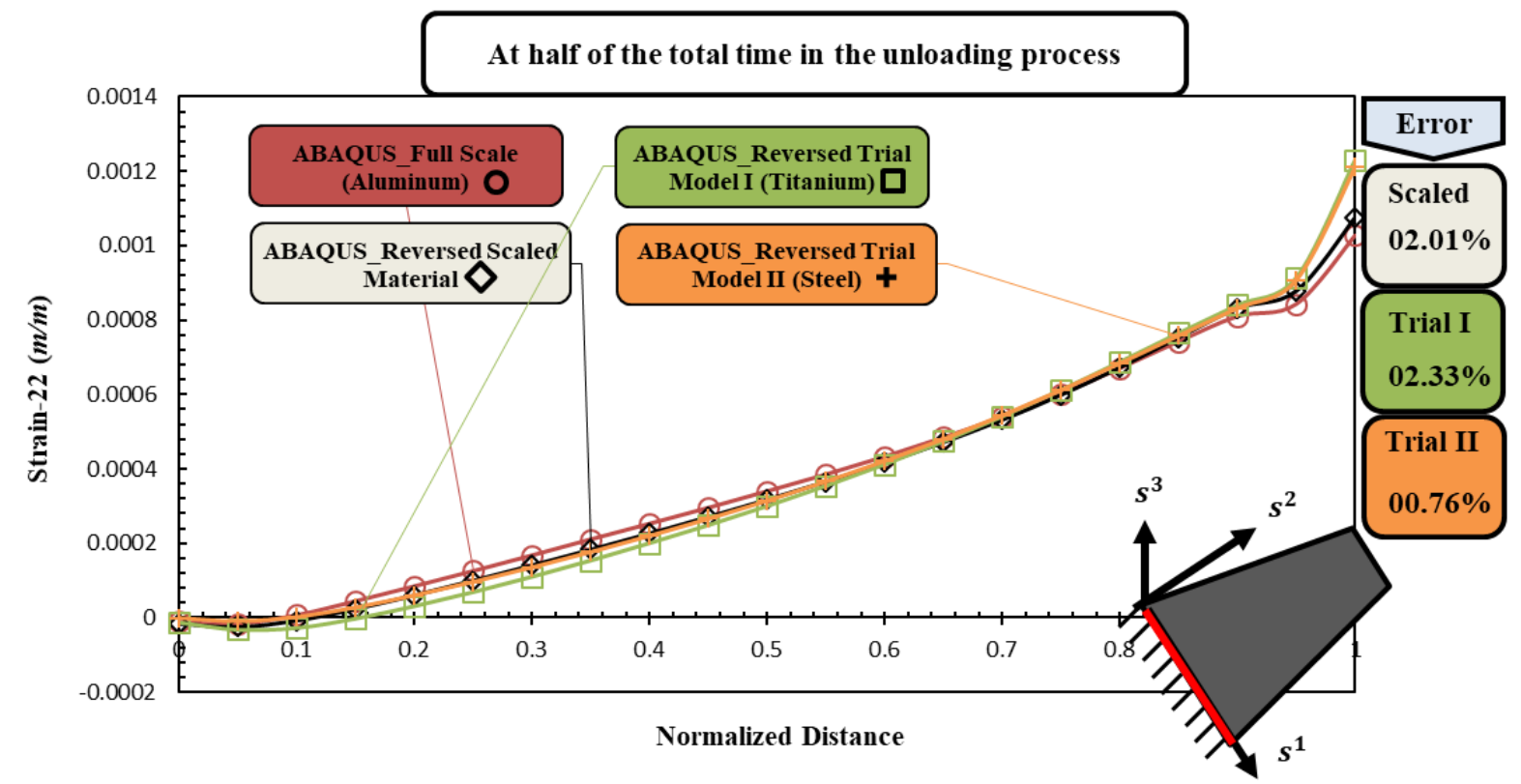

(d) $\varepsilon^{22}$

Fig. 14. Elastodynamic deformation of full-scale and reversed airplane wings

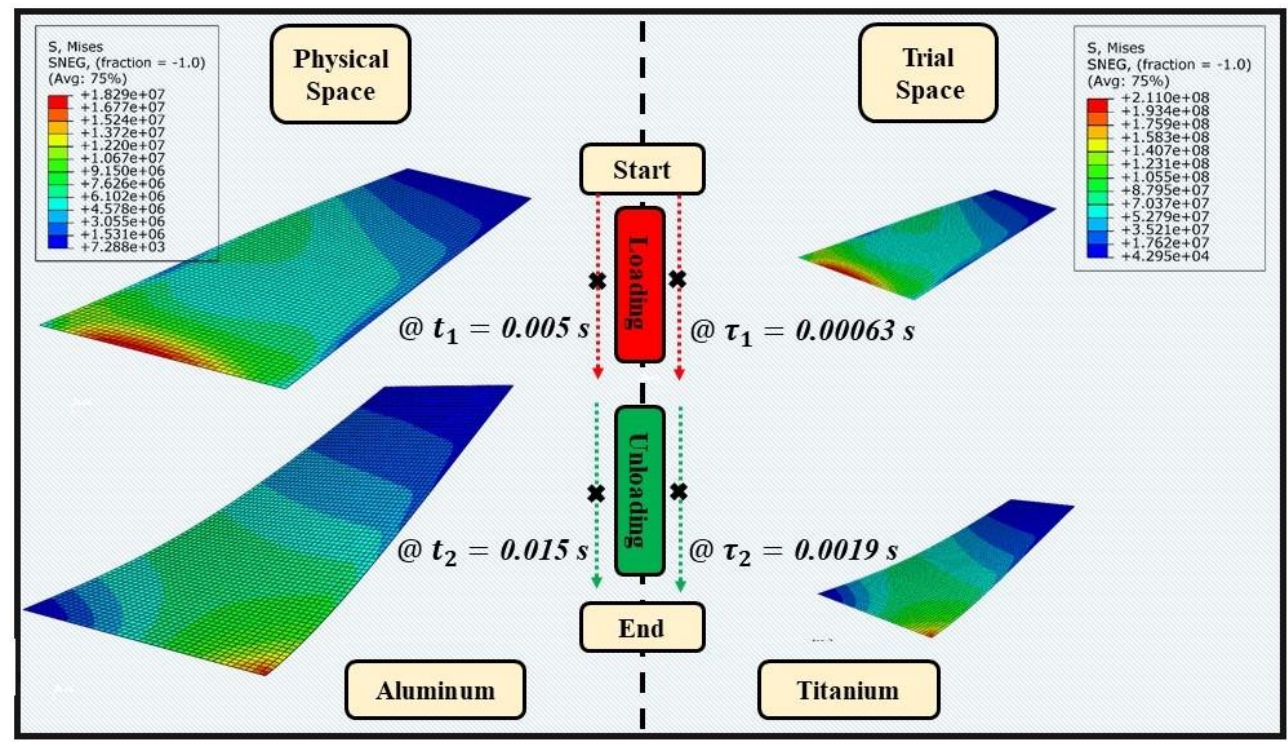

Fig. 15. A comparison between the von Mises stress distribution of full-scale and first trial airplane wings at two different times

\subsection{Part C: Equivalent Isotropic Models for Orthotropic Materials}

It is shown here that the thermal and elastic deformational response characteristics of a structure made of an orthotropic material can be obtained using a structure made of an isotropic material using the similitude theory. Two studies are performed with the first one concerned with temperature distribution for specified boundary and initial temperatures and the second one about elastic deformation of a fully-clamped rectangular plate subjected to a transverse dynamic pressure loading.

\subsubsection{Case Study C.I: Pure Thermal Process}

Obtained here numerically is the temperature distribution of a structure made of an orthotropic thermal conductivity material using a trial model made of an isotropic thermal conductivity material in which the dimensional scaling factors cannot be selected freely. It is 
shown here that the different thermal conductivities in different directions can be scaled to a single thermal conductivity and thus providing an equivalent model made of an isotropic material but representing the behaviour of an aniso/orthotropic structure. The in-house finite element code based algorithm presented in Ref. [31] is employed for determining the transient thermal behaviour of the considered case studies in which a mesh of 32003 -node linear heat transfer triangle elements are used for the considered specimens. Also, fixed time increments $0.005 \mathrm{~s}$ are, where the numerical time integration is based on the backward Euler technique. Also the finite element software Abaqus with transient heat transfer solver [30] is employed for verification of the full-scale model results. Aluminium rectangular honeycomb having $a_{p s}$ and $b_{p s}$ (according to Fig. 16) equal to $0.200 \mathrm{~m}$ and $0.200 \mathrm{~m}$ with boundary and initial temperatures $700 \mathrm{~K}$ and $300 \mathrm{~K}$ is considered as the full-scale model. This honeycomb can be replaced by a rectangular plate which has orthotropic thermal conductivity properties [40] according to Fig. 16. The density, thermal conductivities in $s^{1}$ and $s^{2}$ directions and specific heat capacity of the rectangular plate are respectively $2710 \mathrm{~kg} / \mathrm{m}^{3}, 1.449 \mathrm{~W} / \mathrm{m} . \mathrm{K}$, $2.126 \mathrm{~W} / \mathrm{m} . \mathrm{K}$ and $896 \mathrm{~J} / \mathrm{kgK}$ [40]. Aluminium with properties tabulated at Table 2 is used as the material of the first trial model. The scaling factors and the equations for calculating them are listed in Table 7. The scaled model and first trial model dimensions are obtained as $a_{t s}=1.755 \mathrm{~m}$ and $b_{t s}=1.449 \mathrm{~m}$. It transpires that the scaled material properties obtained provide a complete match with the aluminium properties. It is expected therefore that the temperature distribution of the full-scale model can be predicted with zero error by the trial model; this is investigated by the FE numerical simulation. Additionally a rectangular plate made of steel with properties tabulated at Table 2 is used as the second trial model. A schematic diagram of full-scale and trial models is presented in Fig. 16.

The temperature distribution of full-scale model which has orthotropic thermal conductivity properties and the reversed temperature distribution of scaled model, trial model I and trial model II are compared on paths along the $s^{1}$ and $s^{2}$ axes according to Figs. 17 and 18 at two different instances in time. It is revealed that the full-scale model response is predicted with zero error by both of trial models I and II.

Table 7. Scaling factors of case study C.I

\begin{tabular}{|c|c|c|c|c|c|c|}
\hline & $g$ & $\beta_{1}$ & $\beta_{2}$ & $\alpha^{\rho}$ & $\alpha^{s}$ & $\alpha^{e}$ \\
\hline $\begin{array}{l}\text { Trial Model I } \\
\text { (Aluminum) }\end{array}$ & 0.800 & 8.775 & 7.244 & 0.015 & 0.016 & 0.010 \\
\hline $\begin{array}{l}\text { Trial Model II } \\
\text { (Steel) }\end{array}$ & 0.800 & 4.005 & 3.306 & 0.026 & 0.049 & 0.017 \\
\hline Equation & - & $\begin{array}{c}\beta_{1}=\sqrt{\left(\frac{g \rho_{p s}}{\rho_{t s}}\right)\left(\frac{K_{x_{1} t s} c_{p p s}}{K_{s_{1} p s} c_{p t s}}\right)} \\
\text { (see Appendix B.2) }\end{array}$ & $\begin{array}{c}\beta_{2}=\sqrt{\left(\frac{g \rho_{p s}}{\rho_{t s}}\right)\left(\frac{K_{x_{2} t s} c_{p p s}}{K_{s_{2} p s} c_{p t s}}\right)} \\
\text { (see Appendix B.2) }\end{array}$ & $\begin{array}{c}\alpha^{\rho}=\left(\frac{1}{\beta_{1} \beta_{2}}\right)\left(\frac{\rho_{p s}}{\rho_{t s}}\right) \\
\text { from relation } \\
\rho_{p s}=\alpha^{\rho} \rho_{t s} J \\
\text { (see Table 1) }\end{array}$ & $\begin{array}{l}\alpha^{s}=\alpha^{\rho}\left(\frac{c_{p p s}}{c_{p t s}}\right) \\
\text { from relations } \\
\alpha^{\rho} u_{p s}=\alpha^{e} u_{t s} \\
\quad \text { and } \\
\alpha^{s} T_{p s}=\alpha^{e} T_{t s} \\
\text { (see Section 5) }\end{array}$ & $\begin{array}{c}\alpha^{e}=\left(\frac{g^{2}}{\beta_{1} \beta_{2}}\right)\left(\frac{\rho_{p s}}{\rho_{t s}}\right) \\
\text { (see Section 5) }\end{array}$ \\
\hline
\end{tabular}




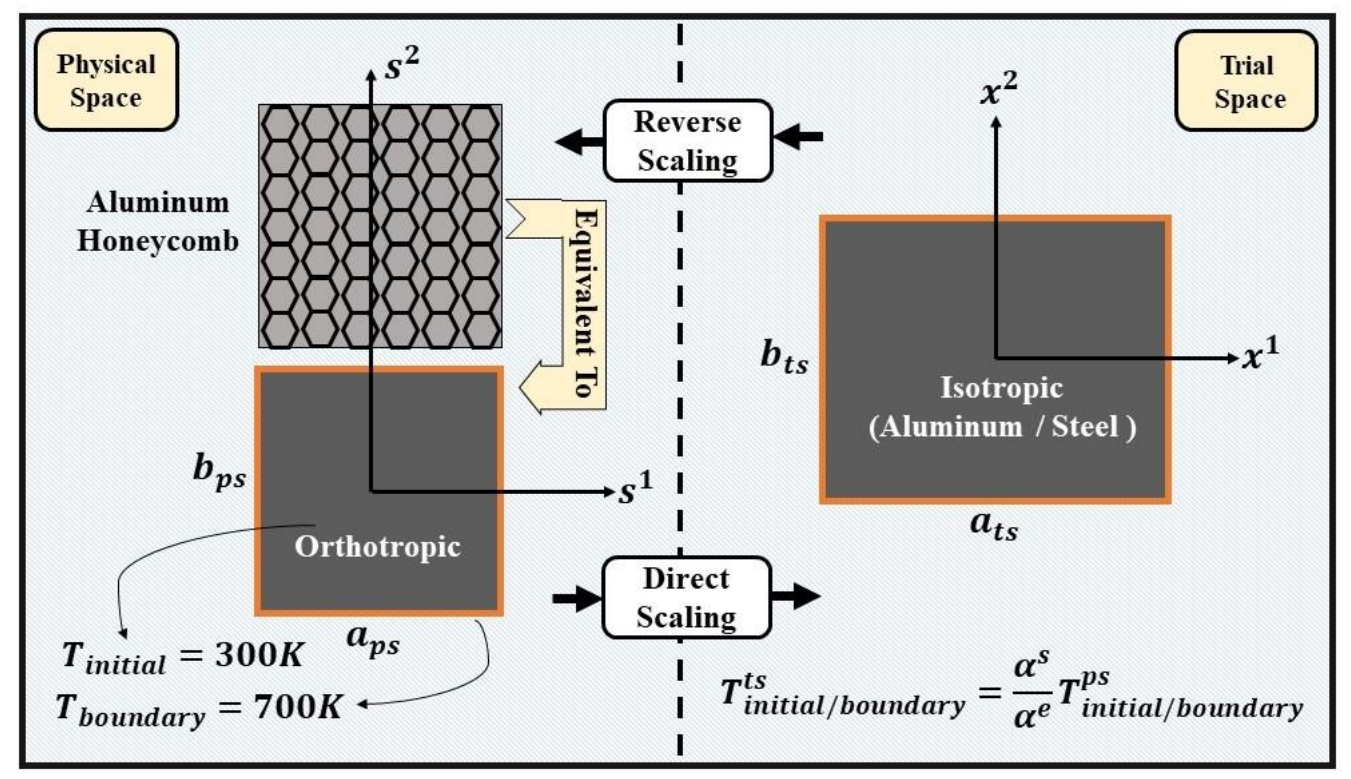

Fig. 16. A schematic diagram representing the scaling of an orthotropic thermal conductivity to an isotropic one for case study C.I

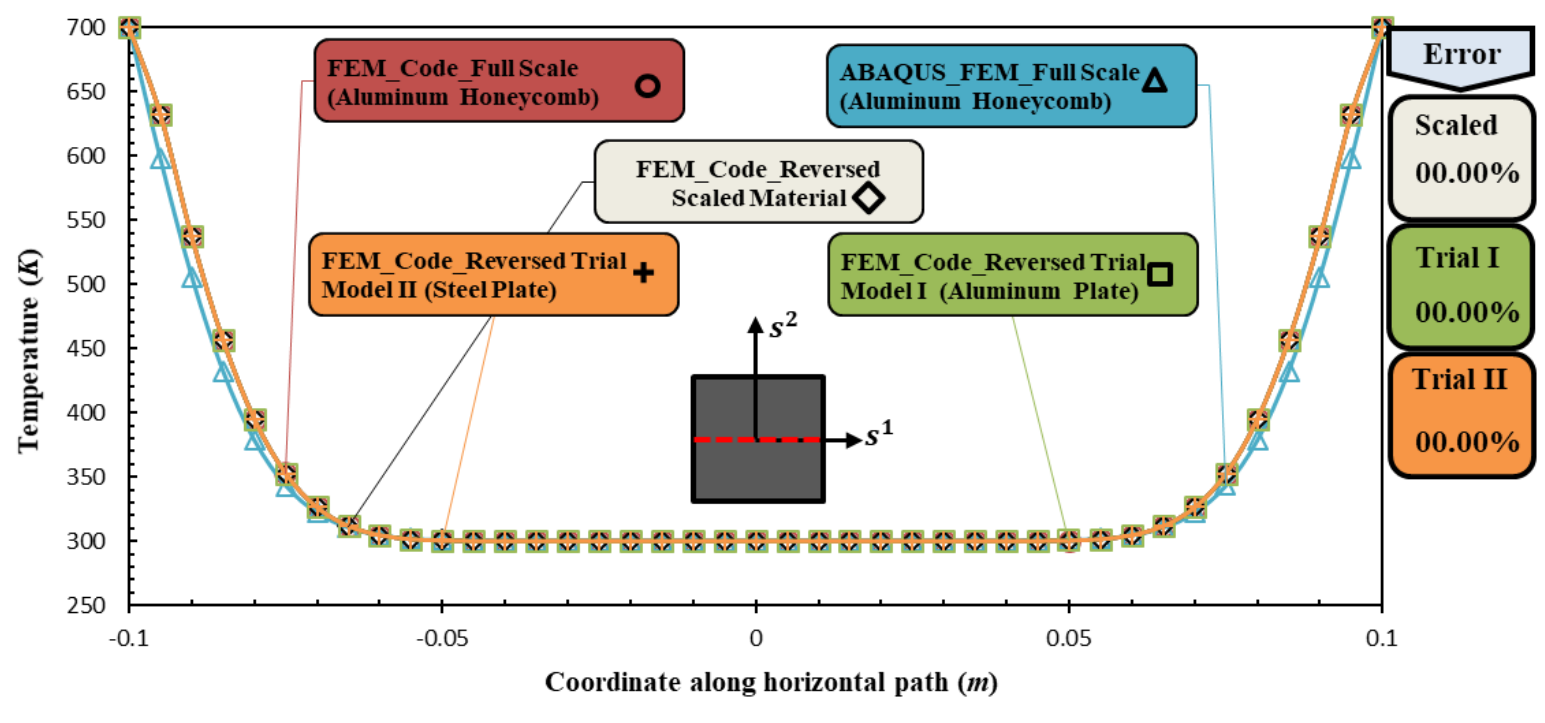

(a) $200 \mathrm{~s}$ 


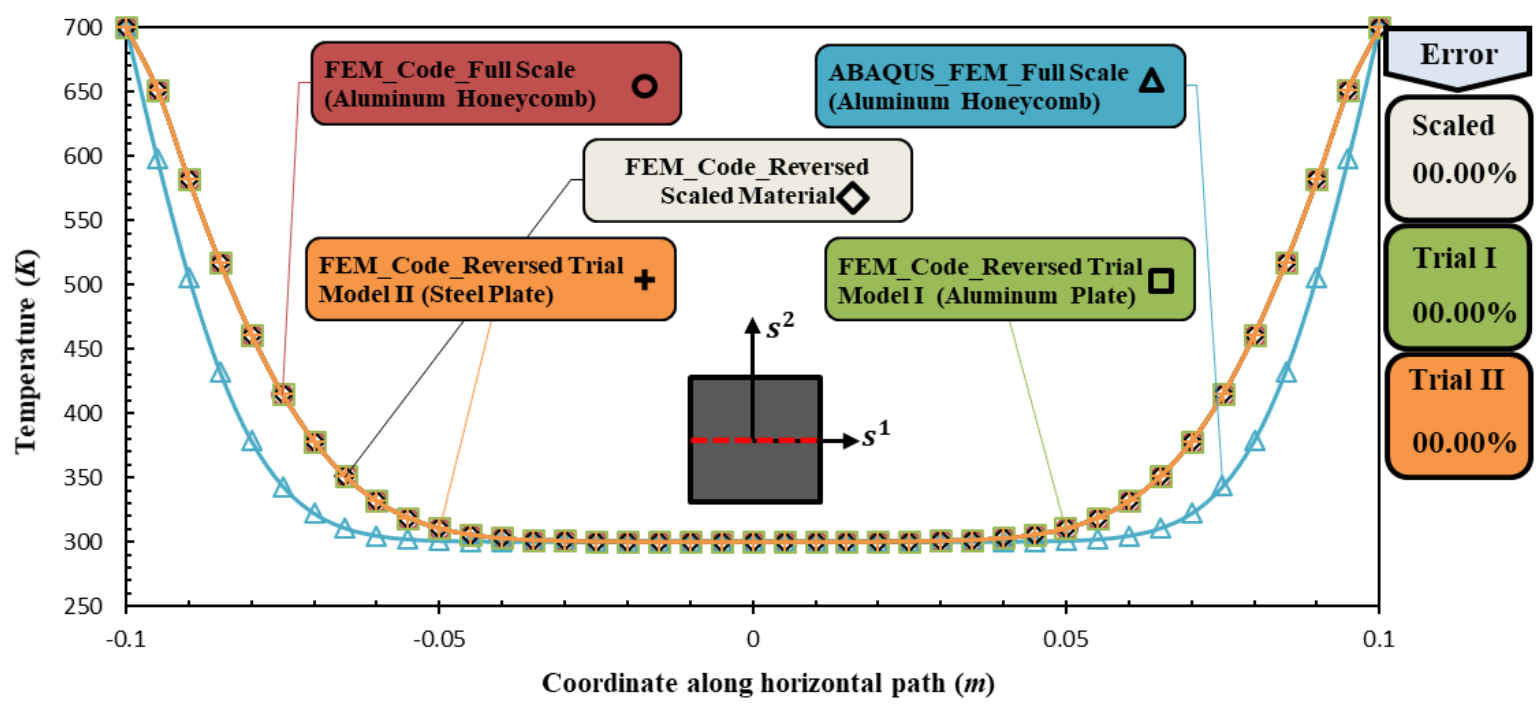

(b) $400 \mathrm{~s}$

Fig. 17. Temperature distribution of a plate made of an orthotropic thermal conductivity material predicted by plates made of isotropic thermal conductivity materials along a path labelled by the $s^{1}$-axis for two different times

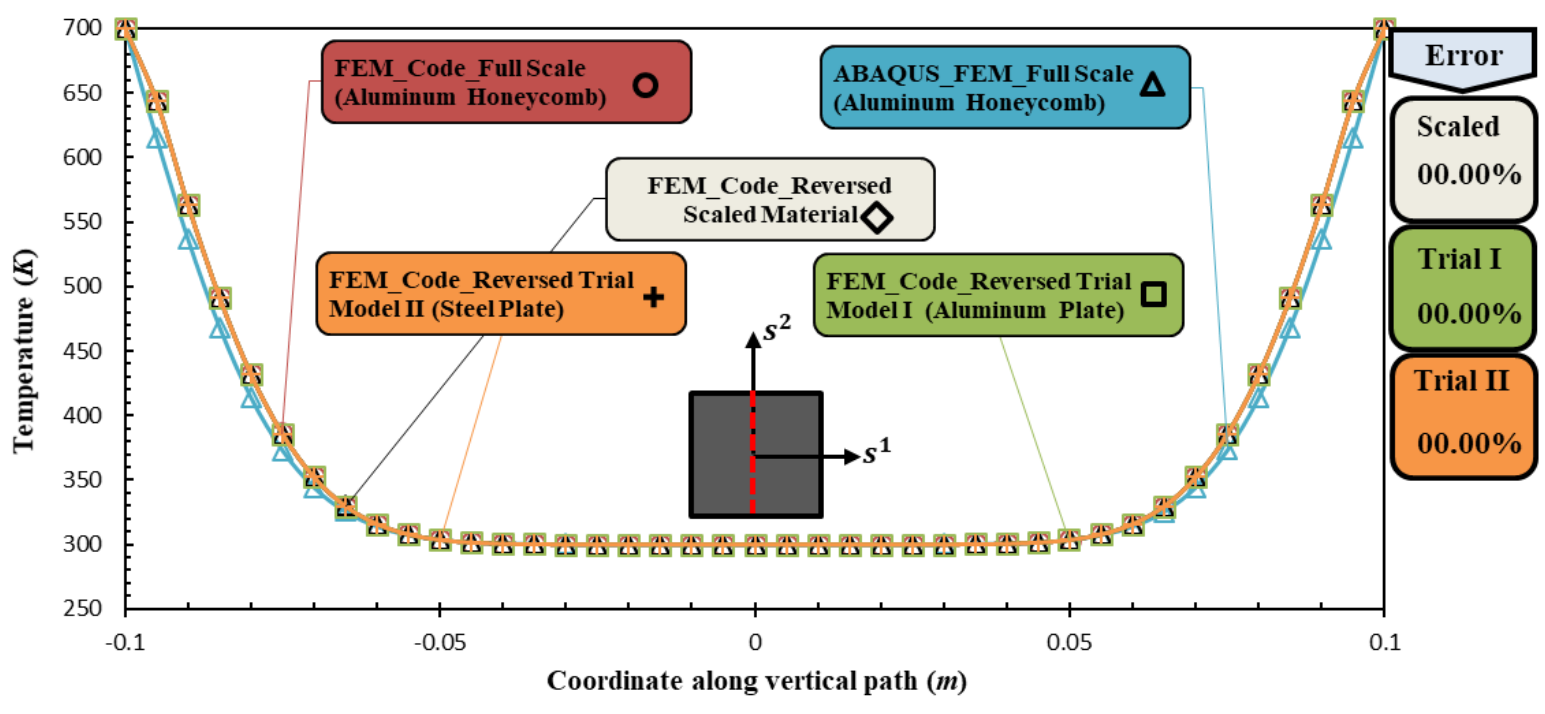

(a) $200 \mathrm{~s}$ 


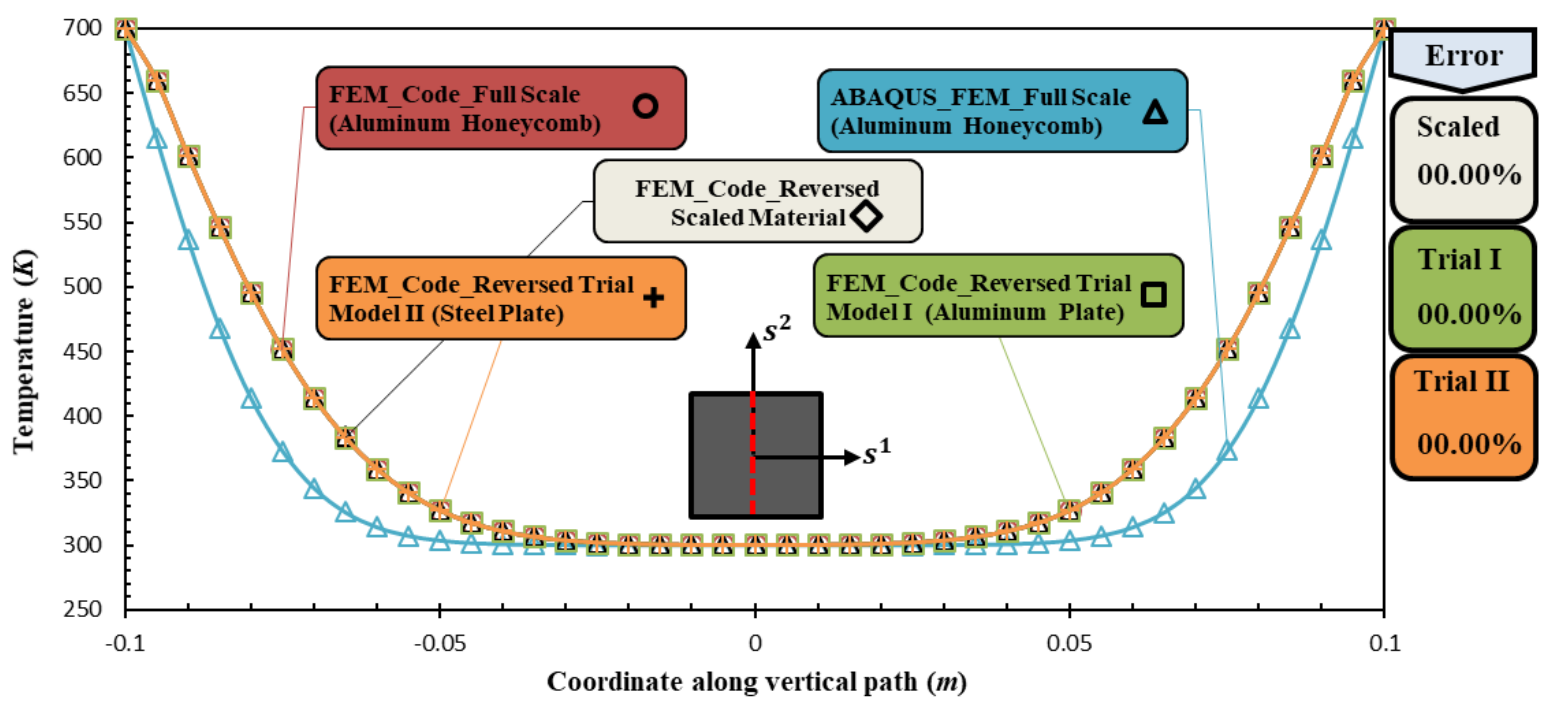

(b) $400 \mathrm{~s}$

Fig. 18. Temperature distribution of a plate made of an orthotropic thermal conductivity material predicted by plates made of isotropic thermal conductivity materials along a path labelled by the $s^{2}$-axis for two different times

\subsubsection{Case Study C.II: Elasto-Dynamic Deformation}

Shown here numerically is that the response characteristics of a structure made of an orthotropic material subjected to a dynamic loading can be obtained using a trial model made of an isotropic material. The numerical simulation is performed using the commercial finite element software Abaqus [30] by employing the explicit solver in which $400 \mathrm{~S} 4 \mathrm{R}$ elements are used for discretization of considered structure.

A fully-clamped rectangular plate made of Graphite/Epoxy material with properties presented in Sections 9.1.2 and 9.1.3 also is considered here as the full-scale model. The used Graphite/Epoxy material [41] has density of $1600 \mathrm{~kg} / \mathrm{m}^{3}$ and also its material properties are determined in Voigt form as

$$
\left[\begin{array}{ccccc}
181.811 & 2.897 & 0 & 0 & 0 \\
2.897 & 10.346 & 0 & 0 & 0 \\
0 & 0 & 3 & 0 & 0 \\
0 & 0 & 0 & 7 & 0 \\
0 & 0 & 0 & 0 & 7.170
\end{array}\right] \times 10^{9} \mathrm{~Pa}
$$

Additionally a rectangular plate made of aluminium with properties tabulated at Table 2 is considered as the first trial model. The scaling factors and used equations for determining of them are presented according to Table 8 . Therefore, the dimensions of rectangular plate is obtained equal with $a_{t s}=0.122 \mathrm{~m}, b_{t s}=0.357 \mathrm{~m}$ and $t_{t s}=7.500 \times 10^{-4} \mathrm{~m}\left(a_{t s}, b_{t s}\right.$ and $t_{t s}$ are presented in Fig. 19) for scaled model and trial model I and also their transverse dynamic pressure is obtained equal with $37887 \mathrm{~Pa}$. Also, the density of scaled material is obtained 
equal with $2770 \mathrm{~kg} / \mathrm{m}^{3}$ and the properties of scaled material are determined using Eq. (B2) in Voigt form as

$$
\left[\begin{array}{cccrl}
78.555 & 10.633 & 0 & 0 & 0 \\
10.633 & 322.569 & 0 & 0 & 0 \\
0 & 0 & 16.530 & 0 & 0 \\
0 & 0 & 0 & 4.541 & 0 \\
0 & 0 & 0 & 0 & 26.316
\end{array}\right] \times 10^{9} \mathrm{~Pa}
$$

A rectangular plate made of steel material with properties listed at Table 2 is considered as the second trial model. A schematic diagram of full-scale and trial models is presented according to Fig. 19. Temporal displacement in $s^{3}$ direction, $\sigma^{11}, \sigma^{22}, \varepsilon^{11}$ and $\varepsilon^{22}$ of full-scale and reversed scaled and trial models are compared in the centre of rectangular plate according to Fig. 20. It is revealed that the response characteristic of the full-scale model (made of an orthotropic material) suffers some inaccuracy arising from a combination of modelling errors and material mismatch. It is generally always possible however by means of alternative designs with different materials and adjusted parameter settings to achieve greater accuracies. However, no attempt is made in this study to do an exhaustive search for different materials in an attempt to minimise the errors involved. Practical choices are being made using standard materials and the results of the study and the errors involved reflect the constraints applied. The objective here is to simply demonstrate the new concepts on a range of problems using a small sample of materials.

Table 8. Scaling factors for case study C.II

\begin{tabular}{|c|c|c|c|c|}
\hline & $\beta_{1}$ and $\beta_{2}$ & $\beta_{3}$ & $g$ & $\alpha^{\rho}$ \\
\hline $\begin{array}{c}\text { Trial Model I } \\
\text { (Aluminium) }\end{array}$ & 0.612 and 1.784 & 0.750 & 1 & 0.705 \\
\hline $\begin{array}{c}\text { Trial Model II } \\
\text { (Steel) }\end{array}$ & 0.611 and 1.819 & 0.750 & 1 & 0.245 \\
\hline Equation & $C_{t s}^{1111}=\left(\frac{|F|^{-2 / D} \beta_{1}^{4}}{k}\right) C_{p s}^{1111}$ & - & - & $\alpha^{\rho}=\left(\frac{1}{\beta_{1} \beta_{2} \beta_{3}}\right)\left(\frac{\rho_{p s}}{\rho_{t s}}\right)$ \\
and \\
from relation \\
$\rho_{p s}=\alpha^{\rho} \rho_{t s} J$ \\
(see Table 1$)$
\end{tabular}




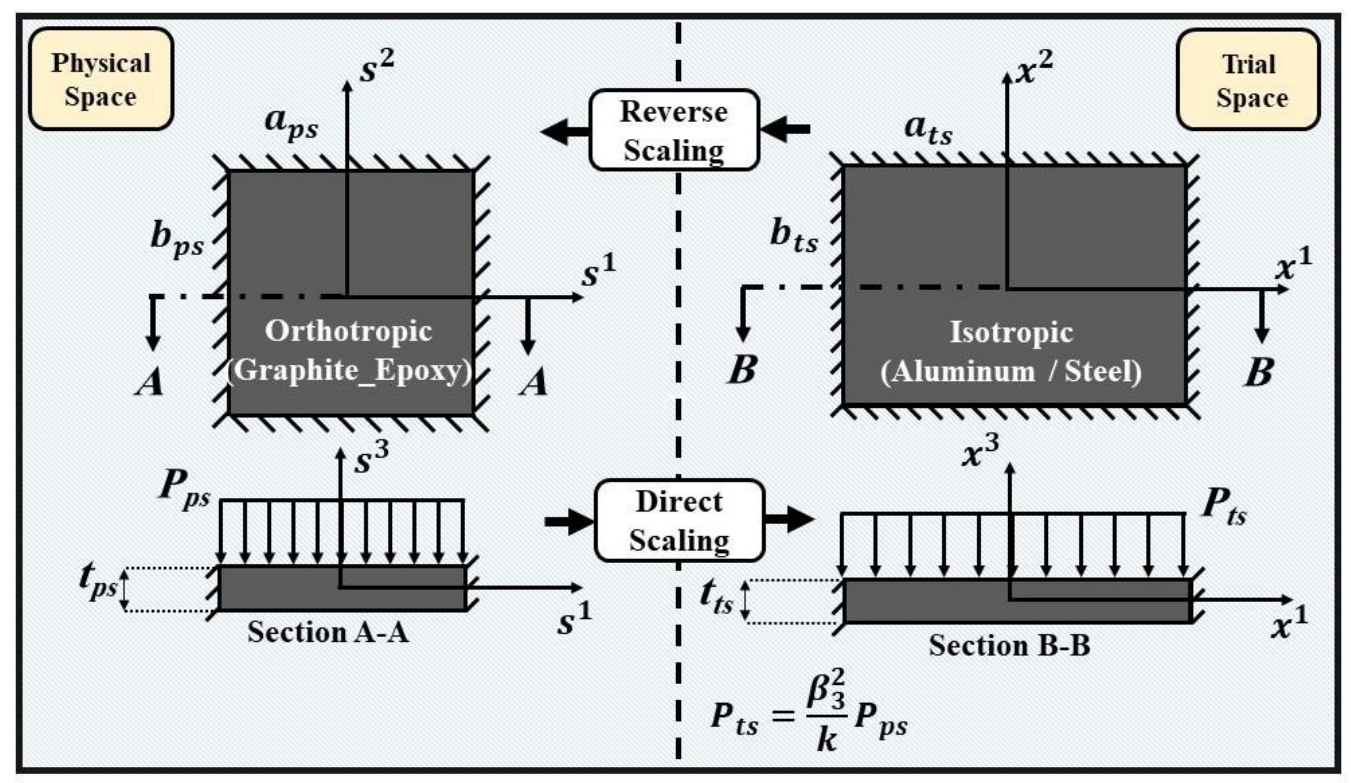

Fig. 19. A schematic diagram representing the scaling of a plate made of an orthotropic material to an isotropic one for case study C.II

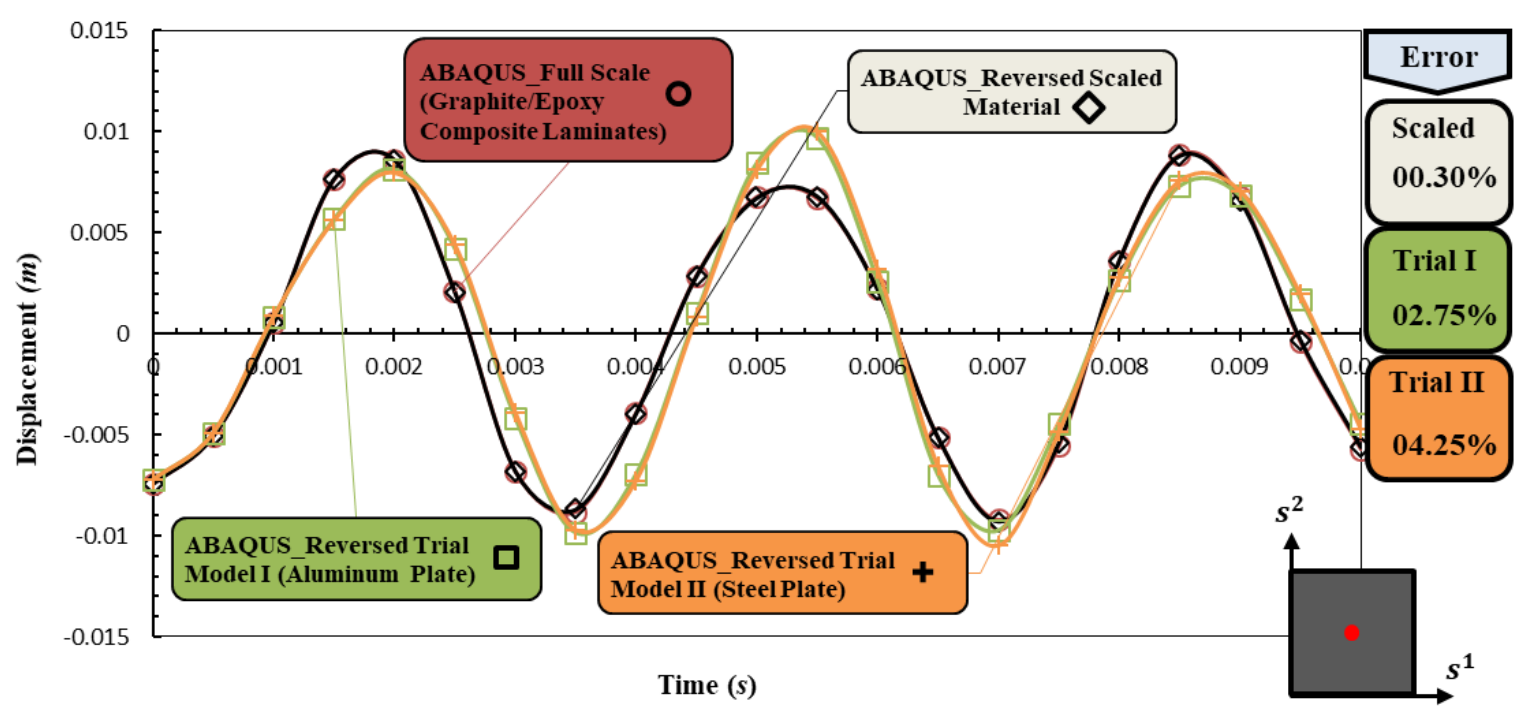

(a) Deflection in $s^{3}$-direction 


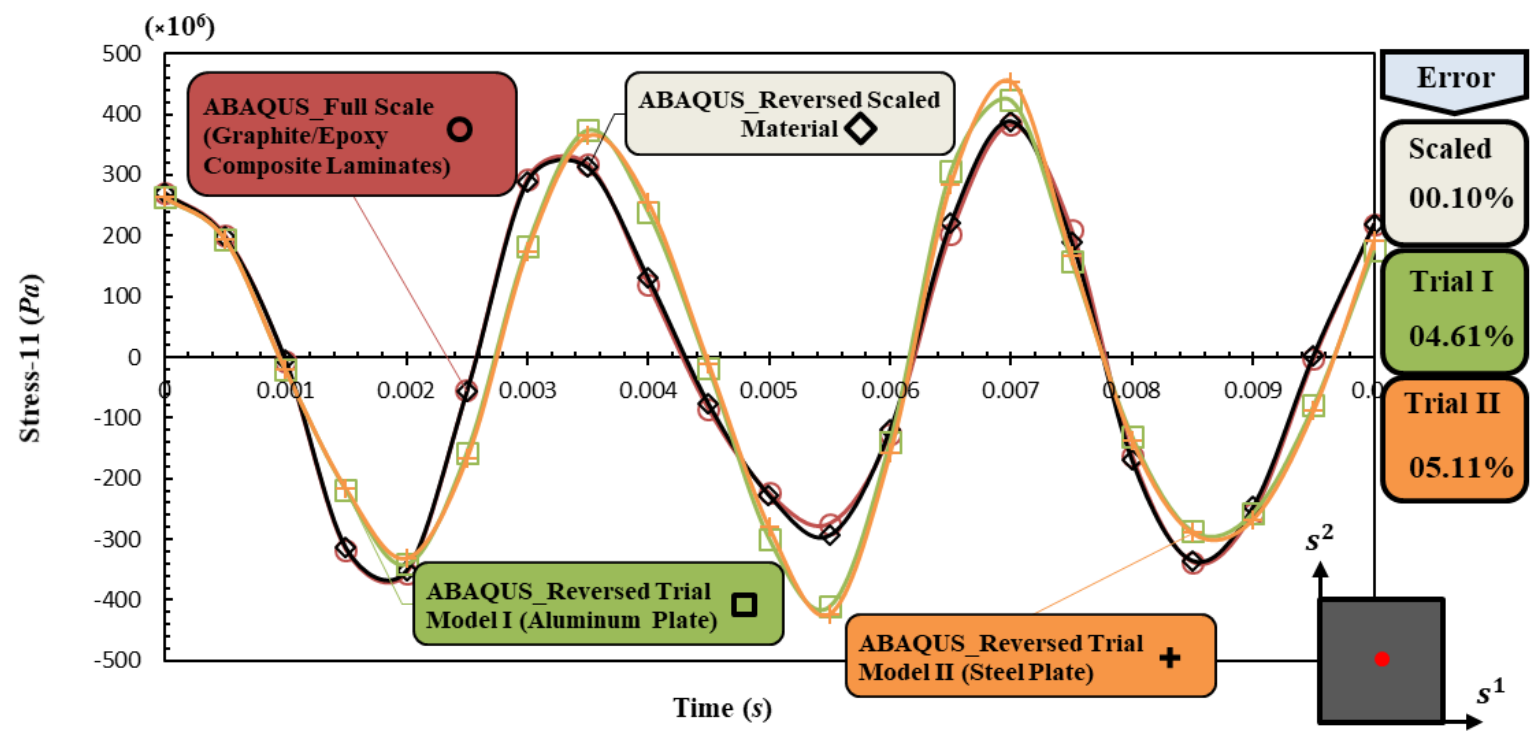

(b) $\sigma^{11}$

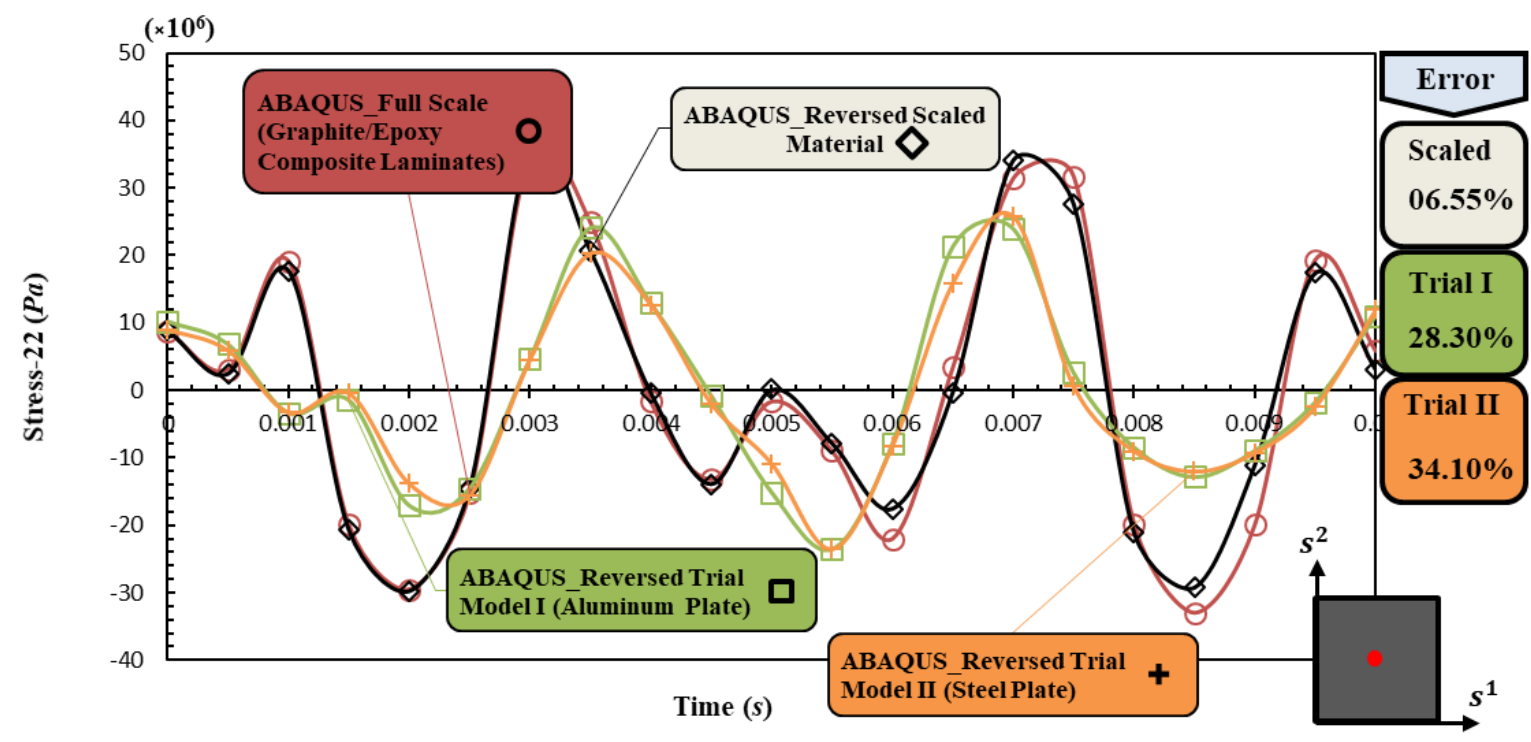

(c) $\sigma^{22}$ 


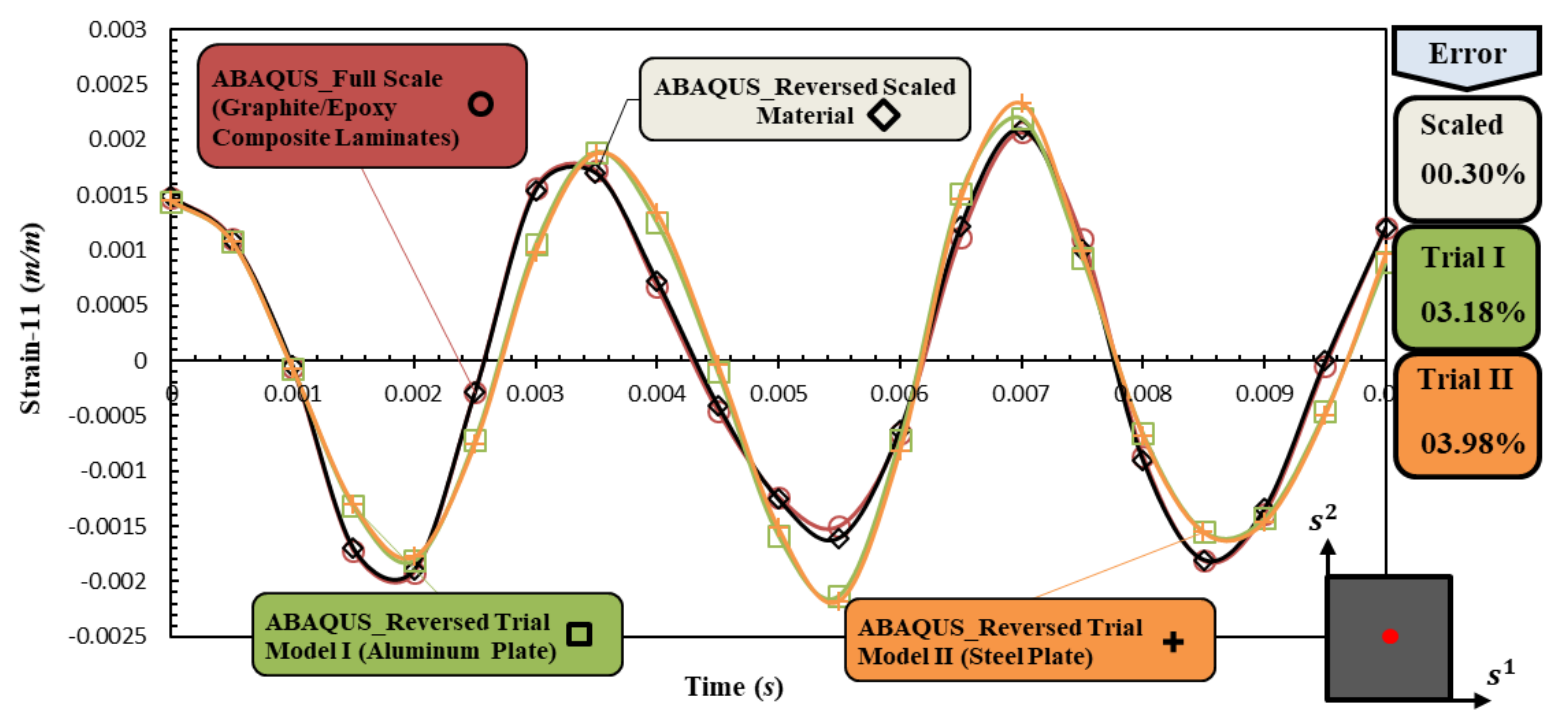

(d) $\varepsilon^{11}$

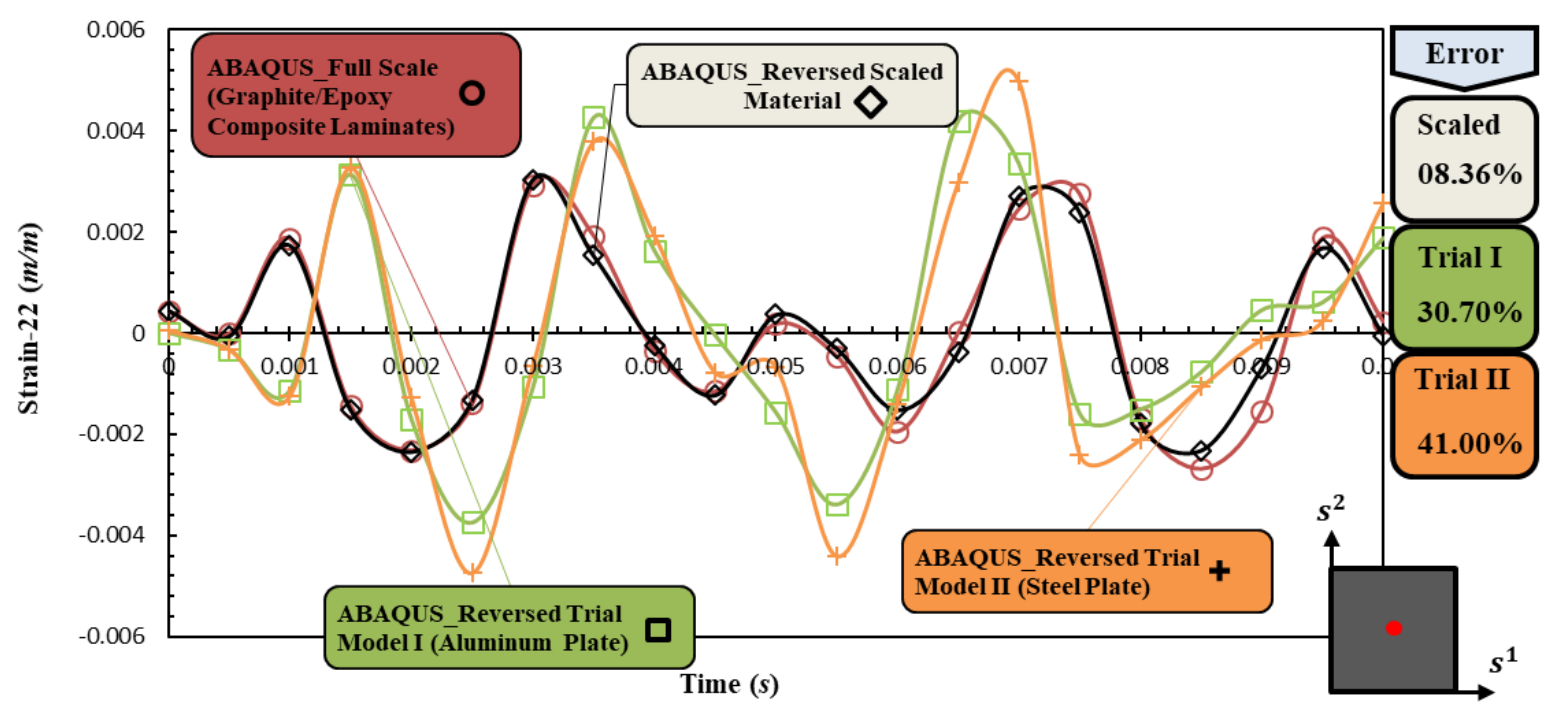

(e) $\varepsilon^{22}$

Fig. 20. Elastodynamic deformation of a plate made of an orthotropic material predicted by plates made from isotropic materials at the centre of the rectangular plate

\section{CONCLUSIONS}

This paper examined the possibility that geometric similarity could be broken yet still retain a form of similarity that facilitated the creation of physical models. It was demonstrated in the paper that by examining the affect anisotropic-space contraction had on transport equations that:

- Proportional transport equations were possible for fully coupled thermomechanics but necessitated the use of metric free products, which lead to the establishment of the law of finite similitude for anisotropic scaling.

- Constitutive equations for thermal and elastic behaviour could be obtained in metricfree form but raising the index for displacement introduced an unwelcome (non- 
physical) metric into the momentum transport equation. This observation provided three outcomes:

- Elastic quasi-static deformation does not require the displacement index to be raised (since no inertial term present) and consequently physically realisable finite similitude is achieved.

- Rigid-body dynamics does not involve the elastic constitutive equation and again physically realisable finite similitude is achieved.

- Physically realisable finite similitude was shown possibly for mechanics involving a dominant component of velocity/displacement. Although the metric was present for this case its approximation was shown possibly, which introduced a particular form of modelling error termed similitude error.

- The numerical trials confirmed:

o The theory's application is relatively straightforward.

- Thermal analysis on an elliptic shape (made of titanium) could be performed on a circular shape (made of aluminium).

- Plate and wing structures subjected to static or dynamic loading could be scaled anisotropically with different materials used for full-scale and trial models.

- The thermal response of an orthotropic material could be predicted to high accuracy with a model with isotropic material.

- The elastic response of a structure subjected to a static or dynamic loading and made of an orthotropic material could be predicted to good accuracy in some field variables using a trial model made of an isotropic material.

\section{REFERENCES}

[1] Sedov L. I., Similarity and Dimensional Methods in Mechanics, CRC Press, Inc, $10^{\text {th }}$ Ed. 1993, ISBN: 0-8493-9308-6.

[2] Tan Q. M., Dimensional Analysis: With Case Studies in Mechanics, Springer; 2011, ISBN-10: 3642448062 .

[3] Ajiboye J. S., Jung K. H., Taek Y., Sensitivity Study of Frictional Behaviour by Dimensional Analysis in Cold Forging, Journal of Mechanical Science and Technology, Vol. 24, pp. 115-118, (2010).

[4] Ezra A. A., Penning F. A., Development of Scaling Laws for Explosive Forming, Experimental Mechanics, Vol. 2, pp. 234-239, (1962).

[5] Zohuri B., Dimensional Analysis and Self-Similarity Methods for Engineers and Scientists, Springer; 2015, ISBN: 978-3-319-13475-8.

[6] Florence A. L., Goodier J. N., Dynamic Plastic Buckling of Cylindrical Shells in Sustained Axial Compressive Flow, Journal of Applied Mechanics, Vol. 35, pp. 80-88, (1968).

[7] Wang A., Tian W., Mechanism of Buckling Development and Strain Reversal Occurrence in Elastic-Plastic Cylindrical Shells under Axial Impact, International Journal of Non-Linear Mechanics, Vol. 43, pp. 722-732, (2008).

[8] Jones N., Structural Impact, Cambridge University Press, $2^{\text {th }}$ Ed. 2012.

[9] Coutris N., Monavon A., Nonlinear Plate Theory: Dimensional Analysis and Asymptotic Formulation of the Large Scale Problem, International Journal of Engineering Science, Vol. 26, pp. 1189-1215, (1988). 
[10] Zohdi T. I., On Simple Scaling Laws for Pumping Fluids with Electrically-Charged Particles, International Journal of Engineering Science, Vol. 123, pp. 73-80, (2018).

[11] Smith E., Fresh Perspectives Regarding Scaling of the Failure of Quasi-Brittle Materials, Vol. 37, pp. 1251-1259, (1999).

[12] Oshiro R. E., Alves M., Scaling Impacted Structures, Archive of Applied Mechanics, Vol. 74, pp. 130-145, (2004).

[13] Alves M., Oshiro R. E., Scaling the Impact of a Mass on a Structure, International Journal of Impact Engineering, Vol. 32, pp. 1158-1173, (2006).

[14] Oshiro R. E., Alves M., Scaling of Cylindrical Shells under Axial Impact, International Journal of Impact Engineering, Vol. 34, pp. 89-103, (2007).

[15] Oshiro R. E., Alves M., Scaling of Structures Subject to Impact Loads When Using a Power Law Constitutive Equation, International Journal of Solids and Structures, Vol. 46, pp. 3412-3421, (2009).

[16] Ogden R. W., Large Deformation Isotropic Elasticity: On the Correlation of Theory and Experiment for Compressible Rubberlike Solids, Proceedings of Royal Society A, Vol. 328, (1972).

[17] Ogden R. W., Large Deformation Isotropic Elasticity - On the Correlation of Theory and Experiment for Incompressible Rubberlike Solids, Proceedings of Royal Society A, Vol. 326, (1972). [18] Elam C. F., Sc D., Tensile Tests on Alloy Crystals. - Part IV - A Copper Alloy Containing Five Percent. Aluminium, Proceedings of Royal Society A, Vol. 116, (1927).

[19] Azdoud Y., Cheng J., Ghosh S., Wavelet-Enriched Adaptive Crystal Plasticity Finite Element Model for Polycrystalline Microstructures, Computer Methods in Applied Mechanics and Engineering, Vol. 327, pp. 36-57, (2017).

[20] Casaburo A., Petrone G., Franco F., De Rosa S., A Review of Similitude Methods for Structural Engineering, Applied Mechanics Reviews, Vol. 71, (2019).

[21] Huntley H. E., Dimensional Analysis, Dover. LOC 67-17978, 1967.

[22] Siano D., Orientational Analysis - A Supplement to Dimensional Analysis - I, Journal of the Franklin Institute, Vol. 320, pp. 267-283, (1985).

[23] Siano D., Orientational Analysis, Tensor Analysis and the Group Properties of the SI Supplementary Units - II, Journal of the Franklin Institute, Vol. 320, pp. 285-302, (1985).

[24] Davey K., Darvizeh R., Al-Tamimi A., Scaled Metal Forming Experiments: A Transport Equation Approach, International Journal of Solids and Structures, Vol. 125, pp. 184-205, (2017).

[25] Moghaddam M., Darvizeh R., Davey K., Darvizeh A., Scaling of the Powder Compaction Process, International Journal of Solids and Structures, Vol. 144-145, pp. 192212, (2018).

[26] Sadeghi H., Davey K., Darvizeh R., Darvizeh A., A Scaled Framework for Strain Rate Sensitive Structures Subjected to High Rate Impact Loading, International Journal of Impact Engineering, Vol. 125, pp. 229-245, (2019).

[27] Sadeghi H., Davey K., Darvizeh R., Darvizeh A., Scaled Models for Failure under Impact Loading, International Journal of Impact Engineering, Vol. 129, pp. 36-56, (2019).

[28] Sadeghi H., Davey K., Darvizeh R., Rajabiehfard R., Darvizeh A., An Investigation into Finite Similitude for High-Rate Loading Processes: Advantages in Comparison to Dimensional Analysis and its Practical Implementation, International Journal of Impact Engineering, Vol. 140, pp. 103554, (2020).

[29] Oden JT, Prudhomme S. Estimation of modeling error in computational mechanics. J Comput Phys, 182(2), pp. 496-515, (2002)

[30] DSSC, Abaqus Theory Manual, Dassault Systemes, Simulia Corp, Providence, RI, USA, (2016).

[31] Bathe K. J., Finite Element Procedures, 2006. 
[32] Lesuer D. R., Experiment Investigations of Material Models for Ti-6Al-4V Titanium and 2024-T3 Aluminum, Technical report, (2000).

[33] Meyers M. A., Dynamic Behavior of Materials, John Wiley \& sons, 1994.

[34] Shackelford J. F., Han Y. H., Kim S., Kwon S. H., Materials Science and Engineering Handbook, CRC Press, $4^{\text {th }}$ Ed. 2015.

[35] Hughes J. R., the Finite Element Method: Linear Static and Dynamic Finite Element Analysis, Courier Corporation, 2012.

[36] Pica A., Hinton E., Efficient Transient Dynamic Plate Bending Analysis with Mindlin Elements, Earthquake Engineering \& Structural Dynamics, Vol. 9, pp. 23-31, (1981).

[37] Schmitt V., Charpin F., Pressure Distributions on the ONERA-M6-Wing at Transonic Mack Numbers, Experimental Data Base for Computer Program Assessment, Report of the Fluid Dynamics Panel Working Group 04, AGARD AR 138, May 1979.

[38] Theodorsen T., Garrick I. E., General Potential Theory of Arbitrary Wing Sections, Report No. 452.

[39] Garrick I. E., Determination of the Theoretical Pressure Distribution for Twenty Airfoils, Report No. 465.

[40] Ogasawara N., Shiratori M., Yu Q., Kurahara T., Estimation of Orthotropic Thermal Conductivity of Honeycomb Material, Heat Transfer, Asian Research, Vol. 31, (2002).

[41] Kaw A. K., Mechanics of Composite Materials, CRC Press, $2^{\text {th }}$ Ed. 2006.

[42] Marsden J. E., Hughes J. R., Mathematical Foundations of Elasticity, Dover Publications, INC. New York, 1983. 


\section{APPENDIX A: METRICS AND ELEMENTAL MEASURE RELATIONSHIPS}

A common tool used in inner-product spaces is the metric, which is a bi-linear operator, which can be used to raise and lower indices and hence conveniently relate quantities in different vector coordinate frames. In the physical space the metrics are trivial being defined by $G_{i j}=\underline{G}_{i} \cdot \underline{G}_{j}=\delta_{i j}$ (and $G^{i j}=\underline{G}^{i} \cdot \underline{G}^{j}=\delta^{i j}$ ) and similarly for the trial space. More interesting is the alternative system for the trial space with metrics $\hat{g}_{i j}=\underline{\hat{g}}_{i} \cdot \underline{\hat{g}}_{j}$ and $\hat{g}^{i j}=\underline{\hat{g}}^{i} \cdot \underline{\hat{g}}^{j}$, which on substitution of $\underline{\hat{g}}^{i}=\underline{g}^{j} \beta\left(F^{-1}\right)_{j}^{i}$ and $\underline{g}_{i}=\beta \underline{\hat{g}}_{j}\left(F^{-1}\right)_{i}^{j}$ from Section 2 gives

$\hat{g}_{i j}=\underline{\hat{g}}_{i} \cdot \underline{\hat{g}}_{j}=\beta^{-2} F^{k}{ }_{i} \underline{g}_{k} \cdot \underline{g}_{\ell} F^{\ell}{ }_{j}=\beta^{-2} F^{k}{ }_{i} \delta_{k \ell} F^{\ell}{ }_{j}=\beta^{-2} F^{k}{ }_{i} F^{k}{ }_{j}$

and

$\hat{g}^{i j}=\underline{\hat{g}}^{i} \cdot \underline{\hat{g}}^{j}=\beta^{2}\left(F^{-1}\right)_{k}^{i} \underline{g}^{k} \cdot \underline{g}^{\ell}\left(F^{-1}\right)_{\ell}^{j}=\beta^{2}\left(F^{-1}\right)_{k}^{i} \delta^{k \ell}\left(F^{-1}\right)_{\ell}^{j}=\beta^{2}\left(F^{-1}\right)_{k}^{i}\left(F^{-1}\right)_{k}^{j}$

or more succinctly in matrix terms $\hat{g}_{\text {cov }}=\beta^{-2} F^{T} F$ and $\hat{g}_{\text {con }}=\beta^{2} F^{-1} F^{-T}$ or in tensorial terms $\underline{\underline{g}}_{\text {cov }}=\hat{g}_{i j} \underline{\hat{g}}^{i} \otimes \underline{\hat{g}}^{j}$ and $\underline{\underline{g}}_{\text {con }}=\hat{g}^{i j} \underline{\hat{g}}_{i} \otimes \underline{\hat{g}}_{j}$.

An alternative relationship to Nanson's first identity for volumes is the Piola formula

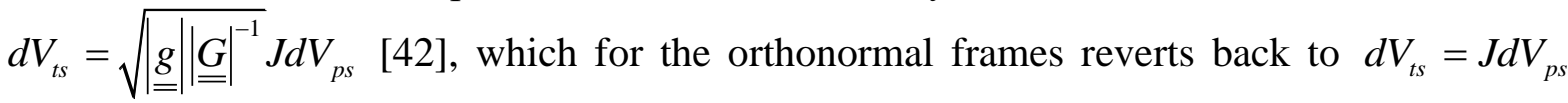
since $|\underline{\underline{g}}|=|\underline{\underline{G}}|=1 . \quad$ Note additionally $\quad \mid \underline{\underline{\underline{g}}}$ cov $|=| \hat{g}_{\text {cov }}|=| \beta^{-2} F^{T} F \mid=1$ and consequently $d \hat{V}_{t s}=d V_{t s}$, which is precisely the result on application of Nanson's first identity directly to $\boldsymbol{d} \hat{\boldsymbol{x}}=\beta F^{-1} \boldsymbol{d} \boldsymbol{x}$. Thus Nanson's first identity and Piola's formula are identical for the transformations considered here.

\section{APPENDIX B: THE EFFECT OF SCALING ON CONSTITUTIVE LAWS}

Consideration in this section is given to small deflection and a Hookean elastic material and heat conduction satisfying Fourier's law of heat conduction.

\section{B.1. Scaling of Hooke's law}

Hooke's constitutive behaviour takes the general tensorial form $\underline{\underline{\sigma}}=\underline{\underline{\underline{C}}}: \underline{\underline{\varepsilon}}$ and is representable in suffix notation $\sigma^{i j}=C^{i j k l} \varepsilon_{k l}$, where the strain component $\varepsilon_{k l}=\frac{1}{2}\left(\partial u_{k} / \partial x^{l}+\partial u_{l} / \partial x^{k}\right)$. The scaling relationship for this law is readily obtainable on application of the contravariant stress and covariant displacement identities listed in Table 1. It can be readily shown that

$\rho_{t s}^{-1} g^{2} \beta^{2} C_{t s}=\rho_{t s}^{-1} F^{-1} F^{-1}: C_{p s}: F^{-T} F^{-T}$

which in suffix notation takes the form

$$
\rho_{t s}^{-1} g^{2} \beta C_{t s}^{i j l k}=\rho_{p s}^{-1}\left(F^{-1}\right)_{i^{\prime}}^{i}\left(F^{-1}\right)_{j^{\prime}}^{j} C_{p s}^{i^{\prime} l^{\prime} k^{\prime}}\left(F^{-T}\right)_{k^{\prime}}^{k}\left(F^{-T}\right)_{l^{\prime}}^{l}
$$


where it is apparent that $C_{t s}$ satisfies the expected symmetry conditions, i.e. $C_{t s}^{i j l k}=C_{t s}^{j i l k}=C_{t s}^{i j k l}=C_{t s}^{l k i j}$.

\section{B.2. Scaling of Fourier's law}

Other material properties of interest to thermo-mechanical analysis is thermal conductivity and with application of a classical Fourier law $\underline{q}_{p s}=-\underline{\underline{K}}_{p s} \cdot \nabla_{p s} T_{p s}$ but for similarity $\rho_{p s}^{-1} \boldsymbol{q}_{p s}=\rho_{t s}^{-1} g^{3} F^{-1} \boldsymbol{q}_{t s}$ and $F^{T} \cdot \nabla_{t s}=\nabla_{p s}$, and since $\alpha^{s} T_{t s}=\alpha^{e} T_{p s}$, it follows that

$K_{p s}=\frac{\rho_{p s} g^{3}}{\rho_{t s}} \frac{\alpha^{s}}{\alpha^{e}} F^{-1} K_{t s} F^{-T}=g \alpha^{s} J F^{-1} K_{t s} F^{-T}$

with $K_{t s}$ endowed with the symmetric form expected. 